\title{
APPLICATIONS OF MODEL SYSTEMS TO EXPLORE EXCHANGE-CORRELATION EFFECTS IN DENSITY-FUNCTIONAL THEORY
}

THEORY (n)

A thesis presented to

the Faculty of the Graduate School

at the University of Missouri

In Partial Fulfillment

of the Requirements for the Degree

Doctor of Philosophy

by
EDWARD A PLUHAR III

Dr. Carsten Ullrich, Thesis Supervisor

DEC 2020 
The undersigned, appointed by the Dean of the Graduate School, have examined the dissertation entitled:

\section{APPLICATIONS OF MODEL SYSTEMS TO EXPLORE EXCHANGE-CORRELATION EFFECTS IN DENSITY-FUNCTIONAL THEORY}

presented by Edward A Pluhar III, a candidate for the degree of Doctor of Philosophy and hereby certify that, in their opinion, it is worthy of acceptance.

Dr. Carsten Ullrich

Dr. Giovanni Vignale

Dr. Sashi Satpathy

Dr. Rainer Glaser 


\section{ACKNOWLEDGMENTS}

Upon entering the University of Missouri, the thought that I might be doing computational, theoretical physics was somewhat absurd. Not only did theory, in

general, not appeal to me, but my computational prowess was limited to computing sums and calculating magnetic fields in Microsoft Excel. Sitting with Carsten and discussing what he did, was doing, and hoped to accomplish in the future, he gave me a simple project that proved difficult for my meager skills.

That system, the Hubbard Dimer, would become an all-to-familiar friend to me, but at that point, I couldn't see that all paths led back to the start. I owe Dr. Carsten Ullrich enormous amounts of gratitude for his direction down this path with expert advice and opinions. Without his vision, time spent debugging simple errors, and thorough explanations of exchange-correlation functionals and the beauty of DFT, my doctorate would still be nothing more than a dream.

In addition to Carsten's tutelage, I have had the opportunity to work with great minds that have offered constructive criticism and numerous solutions. First, I must acknowledge my predecessor for part of this work, Dr. Yonghui Li, who created and developed the particle-hole map. Without the volume of work that he produced, I wouldn't have as firm a grasp on excited state systems and how to calculate and build visualization tools. Second, Nicolas Tancogne-Dejean was instrumental in providing help and support with Octopus and implementing the Slater Functional within its $f_{\mathrm{xc}}$ libraries to explore the effects that exchange-correlation torques had on real systems. Despite focusing on model systems and more fundamental applications, Nicolas' work made it clear that this research can support future and continued research for years 
to come. Finally, I want to acknowledge my groupmates Jared and Matt. From operating system suggestions to discussions about integration and interpolation methods, both of you have been important conduits for helping me grow and learn about physics, in general, and DFT in particular.

Beyond the classroom, however, lies the real challenge in mental fortitude and pursuit. To that end, I would have been unable to accomplish my goals without the love and support of my family and friends. In the darkest of depths and the highest of mountaintops, Lainey, you were beside me, and I am forever grateful. You have driven me to pursue my dreams and achieve things that I always thought were just out of reach. I love you and the successful compilation of this thesis is as attributal to your support as it is to my dedication and hard work. Additionally, I have to thank my mother, father, my step-mother for their continuous support of my education and career path. Without you all, my education would have been out of reach, and graduate school would have never been a thought in my mind. 
TABLE OF CONTENTS

ACKNOWLEDGMENTS ................ ii

LIST OF TABLES $\ldots \ldots \ldots \ldots \ldots \ldots$ viii

LIST OF FIGURES $\ldots \ldots \ldots \ldots \ldots \ldots \ldots \ldots$ ix

ABSTRACT .................. xv

CHAPTER

1 Introduction ....................... 1

Introduction

2 Background and theory: the many-body problem . . . . . . . . 4

2.1 Separating the systems . . . . . . . . . . . . 5

2.1.1 The Born-Oppenheimer Approximation . . . . . . . . . . 6

2.2 Introducing the problem $\ldots \ldots \ldots \ldots \ldots \ldots$

2.2 .1 Observable quantities . . . . . . . . . . . . . . . . . . 11

2.3 Recasting the problem . . . . . . . . . . . . . . . . . . 13

2.3 .1 Variational method . . . . . . . . . . . . . . . . . 13

2.3.2 Functionals and reformulating the many-body Schrödinger equa-

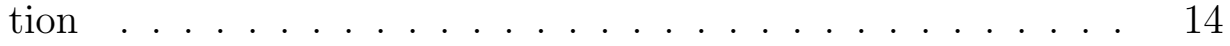

2.3 .3 Noninteracting particles $\ldots \ldots \ldots \ldots$

2.4 Functional approximations and time-dependence . . . . . . . . 22

2.4.1 The exchange-correlation potential . . . . . . . . . 23

2.4 .2 The world of functionals $\ldots \ldots \ldots \ldots$ 
2.4.3 Dynamic systems and time dependence . . . . . . . . . . . . 29

2.5 Bringing it all together: model systems . . . . . . . . . . . . 39

2.5.1 Returning to the quantum world . . . . . . . . . . . . 40

3 The Localized Particle-hole Map . . . . . . . . . . . . . 58

3.1 Introduction . . . . . . . . . . . . . . . . 58

3.2 Formal background . . . . . . . . . . . . . . . . 60

3.2.1 Excitation energies with TDDFT: the Casida equation . . . . 60

3.2.2 Transition density matrix and particle-hole map . . . . . . . 62

3.2.3 Casida equation for localized orbitals . . . . . . . . . . . 64

3.2.4 Localized PHM and metric . . . . . . . . . . . 68

3.3 Results........................... 72

3.4 Conclusions . . . . . . . . . . . . . . . . . . . . 79

4 Exchange-correlation torque effects:

exact comparison via the Hubbard Lattice . . . . . . . . . 82

4.1 Introduction . . . . . . . . . . . . . . . . . 82

4.2 SDFT for the extended Hubbard model with noncollinear magnetic fields 85

4.2.1 Inverting the Kohn-Sham equation in noncollinear spin-DFT . 86

4.2.2 Nearest-neighbor interactions and the zero-torque theorem . . 91

4.3 Nonsymmetric lattice . . . . . . . . . . . . . . . . . . 96

4.3.1 Ground state energy . . . . . . . . . . . . . . 96

4.3.2 Density and magnetization . . . . . . . . . . . . . 97

4.3.3 Hartree and xc potentials and xc magnetic fields . . . . . . . . 100

4.3.4 Exact and approximate xc torques . . . . . . . . . . 103 
4.4 Lattice with $C_{2}$ symmetry $\ldots \ldots \ldots \ldots \ldots$

4.4.1 Ground-state energy, density and magnetization: breaking and enforcing symmetry . . . . . . . . . . . . . 120

4.4.2 xc potentials, magnetic fields, and torques . . . . . . . . 122

4.5 Conclusions . . . . . . . . . . . . . . . . . . . . . 127

5 Spin-orbit coupling and exchange-correlation torque effects in realtime spin-density-functional theory . . . . . . . . 130

5.1 Introduction . . . . . . . . . . . . . . . 130

$5.2 \quad$ Hubbard Trimer . . . . . . . . . . . . . . . . . . . . . . . . . . . . . 132

5.2 .1 Theoretical Background $\ldots \ldots \ldots$. . . . . . . . . 133

5.2 .2 Time-dependence . . . . . . . . . . . . . . . . . . . . . . 135

5.2 .3 Trimer results and discussion $\ldots \ldots \ldots \ldots$

5.3 Time propagation of the tetramer . . . . . . . . . . 145

5.4 Summary and conclusions . . . . . . . . . . . . . . . . . . 148

6 Summary and future direction $\ldots \ldots \ldots \ldots \ldots \ldots \ldots$

6.1 Summary . . . . . . . . . . . . . . . . . . . . . 152

6.2 Future direction . . . . . . . . . . . . . . . 156

6.2.1 Charge and spin transfer in finite systems _ . . . . . . 156

6.2.2 Spin dynamics in noncollinear, spin-frustrated materials . . . . 158

6.2.3 Continued optimization of functionals and inversion methods . 160

6.2.4 Tetramer and Trimer Conclusion _. . . . . . . . . . 162

\section{APPENDIX}

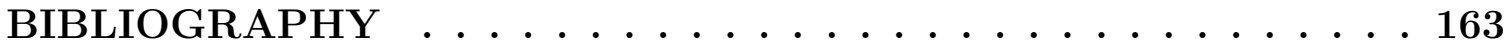




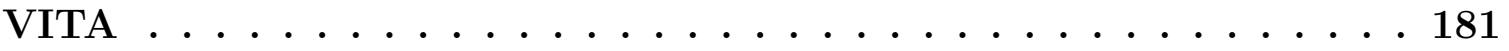




\section{LIST OF TABLES}

Table

3.1 Various descriptors for the PHMs for different excitations: localization number $\bar{M}$, Eq. (3.30), transition localization number $\bar{M}_{t}$, Eq. (3.31), and PHM metric $\tilde{D}_{\Xi}$, Eq. (3.29). $\mathrm{B}_{1}$ and $\mathrm{B}_{4}$ stands for the first and the fourth excitation of the Triple Well B system, respectively. . .

4.1 Exact density and magnetization data for the nonsymmetric and $C_{2^{-}}$ symmetric lattices, in the noninteracting $\left(U_{0}=0\right)$ and strongly corre-

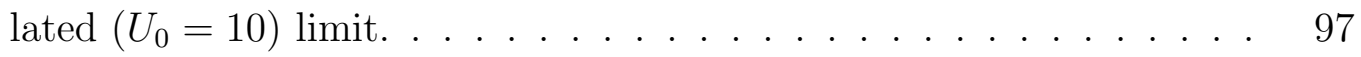




\section{LIST OF FIGURES}

Figure $\quad$ Page

2.1 Exact and calculated Kohn-Sham ground state densities and their cor-

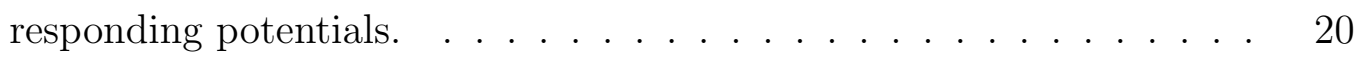

2.2 Time evolution of magnetization integrated around $\mathrm{Ni}$ atoms in a $\mathrm{NiO}$

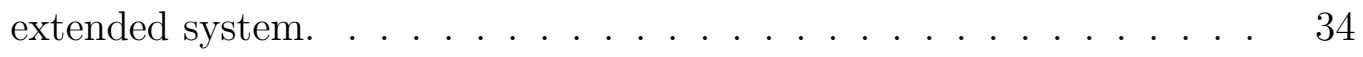

2.3 Current areas of research where quantum dots are being applied or actively explored. . . . . . . . . . . . . . . . . . 41

2.4 The Kronig-Penney potential. . . . . . . . . . . . . . . . . . . 42

2.5 Schematic plots showing (left) discretization of energy levels and (right) how energy dispersion changes with interatomic distance. . . . . . . . 44

2.6 Single-electron capacitance spectroscopy experiment measuring the energy to add an electron to an artificial atom made of GaAs . . . . . 46

2.7 Diagram of quantum dot potential and experimental quantum dot created using GaAs and AlGaAs. . . . . . . . . . . . . . . . 47

2.8 Diagram of the atomic orbitals in a one-dimesional crystal separated

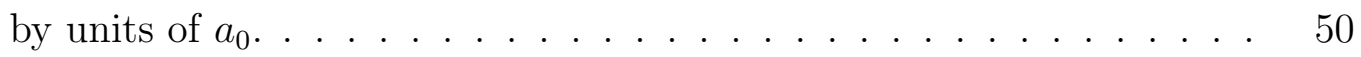

2.9 Schematic of a graphene lattice with A-B sublattice labeling. . . . . . 53 
2.10 Band structure calculations of graphene with spin-orbit coupling for an atomic orbital basis for tight-binding calculations and with densityfunctional theory using a linearized augmented plane-wave basis. . .

2.11 Angle-resolved photoemission spectroscopy measurement of energy bands for graphene with increasing doping of Potassium. . . . . . . . . . . 56

3.1 The energy degenerate molecular orbitals and Lewis structure for $\mathrm{H}_{2} \mathrm{O}$. 66

3.2 The potentials used for the calculations are a single well system (a), a double well system (b), and two triple well systems, labeled as A and B in Table 3.1, respectively (c and d). . . . . . . . . . . . . . 72

3.3 Canonical orbitals for the double well potential, see Fig. 3.2b. Four of the five occupied states are in the deep well, while only one state occupies the shallow right well. States are colored as follows: Lowest blue, 2 - red, 3 - purple, 4 - orange, highest - green. . . . . . . . . . . 73

3.4 The canonical (top) and localized PHM (bottom) for a deep double well potential (Fig. 3.2b). The two PHMs are nearly identical, primarily due to the localized nature of the canonical orbitals prior to applying the localization procedure. . . . . . . . . . . . . . . 73

3.5 The lowest five canonical orbitals (top) and localized orbitals (bottom) for the single well system with ten spin-independent electrons. Lowest state - blue, 2 - red, 3 - purple, 4 - orange, highest - green. . . . . . . 75

3.6 The canonical PHM (top) and the LPHM (bottom) for the lowest excitation of the single well system, with 5 doubly occupied orbitals. $\quad$. 76

3.7 (The canonical PHM (top) and the LPHM (bottom) of the first excitation in the Triple Well B system. . . . . . . . . . . . . . 78 
3.8 The canonical PHM (top) and the LPHM (bottom) of the fourth excitation in the Triple Well B system. . . . . . . . . . . . 78

3.9 The canonical PHM (top) and the LPHM (bottom) of the first excitation in the Triple Well A system. . . . . . . . . . . . . . . . 80

4.1 Flowchart of the CG routine used to calculate the exact KS 4-potential $\vec{v}_{\mathrm{KS}}$ giving rise to a target 4 -density $\vec{m}^{t} \ldots \ldots \ldots \ldots$

4.2 Hubbard tetramer with noncollinear coplanar magnetic field B (blue arrows). (a) Nonsymmetric field: $B_{x, 1}=0.2, B_{x, 2}=0.1, B_{z, 3}=0.3$, $B_{z, 4}=-0.2$. (b) $C_{2}$-symmetric field: $B_{x, 1}=B_{x, 2}=B_{z, 3}=B_{z, 4}=0.1$. In both cases, the scalar potential is $V_{1}=V_{4}=1, V_{2}=V_{3}=-1$. All local external and $\mathrm{xc}$ torques are along the $\pm y$-direction. . . . . . . .

4.3 Ground-state energy of the Hubbard tetramer with nonsymmetric magnetic fields, see Fig. 4.2a, comparing the exact result with various SDFT approximations (see text). The difference between the exact solution and the SCDF approximations are shown in the inset. The lines for XXS and XXSc, as well as STLS and STLSc, respectively, are too close to be distinguished. . . . . . . . . . . . . . . . .

4.4 Density (top) and $x$ - and $z$-components (middle and bottom) of the magnetization of the nonsymmetric Hubbard tetramer. The numbers indicate lattice sites. . . . . . . . . . . . . . . . . . . . . . . 98

4.5 Hartree (top) and xc potential (bottom) of the nonsymmetric Hubbard tetramer. The numbers indicate lattice sites. . . . . . . . . . . . . 101

$4.6 x$ - (top) and $z$-component (bottom) of the xc magnetic field of the nonsymmetric Hubbard tetramer. The numbers indicate lattice sites. 
4.7 Comparison of xc torque $\boldsymbol{\tau}_{\mathrm{xc}, k}$ (red, full lines) and external torque $\boldsymbol{\tau}_{\text {ext }, k}$ (black, dashed lines) of the nonsymmetric tetramer. All torques only have a $y$-component. . . . . . . . . . . . . . . . . . . 104

4.8 Comparison of exact xc torque $\boldsymbol{\tau}_{\mathrm{xc}, k}$ and xc torque approximations with and without torque correction for the nonsymmetric tetramer, on the four lattice sites. All torques only have a $y$-component. . . . . . . . . 105

4.9 (a) Example of a magnetic field with $C_{2}$ symmetry on a 4-point lattice. (b) View along the $y$-axis. . . . . . . . . . . . . . . . . . . . 110

4.10 Ground-state energy of the Hubbard tetramer with $C_{2}$ symmetry. Dashed lines are symmetry enforced calculations, while the solid lines were allowed to break the symmetry of the system. The difference between the exact solution and the corresponding (unrestricted) approximations are plotted in the inset. . . . . . . . . . . . . . . . . 113

4.11 Density (top) and $x$ - and $z$-components (middle and bottom) of the magnetization of the Hubbard tetramer with $C_{2}$ symmetry. The numbers indicate lattice sites. . . . . . . . . . . . . . . . . . 114

4.12 Hartree (top) and xc potential (bottom) of the $C_{2}$-symmetric Hubbard tetramer. The numbers indicate lattice sites. . . . . . . . . . . . . . . 115

$4.13 x$ - (top) and $z$-component (bottom) of the xc magnetic field of the $C_{2}$-symmetric Hubbard tetramer. The numbers indicate lattice sites.

4.14 Comparison of xc torque $\boldsymbol{\tau}_{\mathrm{xc}, k}$ (red, full lines) and external torque $\boldsymbol{\tau}_{\text {ext }, k}$ (black, dashed lines) of the $C_{2}$-symmetric tetramer. All torques only have a $y$-component. . . . . . . . . . . . . . . . . . . 117 
4.15 Comparison of exact xc torque $\boldsymbol{\tau}_{\mathrm{xc}, k}$ and unrestricted and restricted xc torque approximations for the $C_{2}$-symmetric tetramer, on the four lattice sites. All torques only have a $y$-component. . . . . . . . . . . . 118

$4.16 \tau_{\mathrm{xc}}$ 's for $C_{2}$ symmetric system with torque corrections. Torque corrections are only required after the system spontaneously breaks symmetry, as the solutions satisfy the zero-torque theorem prior to breaking symmetry. . . . . . . . . . . . . . . . . . 119

$4.17 x$-component of the magnetization for the nonsymmetric lattice, comparing exact results, Kohn-Sham results with the full $\mathbf{B}_{\mathrm{xc}}$, and KohnSham results with the longitudinal $\mathbf{B}_{\mathrm{xc}}$ (dashed lines). . . . . . . . . . 124

4.18 Same as Fig. 4.17, but for $m_{z, k} \ldots \ldots$. . . . . . . . . . . . 125

4.19 Same as Fig. 4.17, but for the $C_{2}$-symmetric lattice. . . . . . . . . . 125

4.20 Same as Fig. 4.18, but for the $C_{2}$-symmetric lattice. . . . . . . . . . . 126

5.1 Kohn-Sham phase diagram for the Hubbard trimer with a small inplane magnetic field on two lattice sites to lift the degeneracy of the system. . . . . . . . . . . . . . . . . . . 135

5.2 Time-dependent magnetic field applied to the trimer system. The field is a Gaussian pulse applied only in the $x$-direction. . . . . . . . . . . 136

5.3 Chromium trimer showing the noncollinear spin configuration. . . . . 138

5.4 Real-time magnetization precession due to a magnetic pulse along the $x$-axis. The SOC has been set to zero and the hopping constant is set to $0.8 \ldots \ldots \ldots \ldots \ldots \ldots \ldots$ 
5.5 Magnetization differences between the exact magnetizations and KohnSham magnetizations in the $x$-, $y$-, and $z$-directions for the first, second, and third lattice sites with $C=0.4$ and $T=0.2309 \ldots \ldots . . . . .142$

5.6 Magnetization differences between the exact magnetizations and KohnSham magnetizations in the $x^{-}, y$-, and $z$-directions for the first, second, and third lattice sites with $C=0.4$ and $T=0.231 \ldots \ldots . . . . .144$

5.7 Magnetization differences between the exact magnetizations and KohnSham magnetizations in the $x_{-}, y^{-}$, and $z$-directions for the first, second,

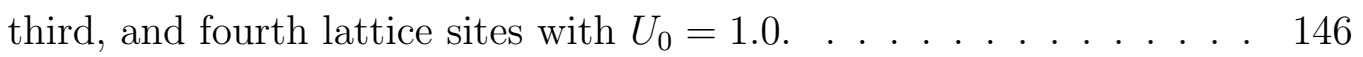

5.8 Magnetization differences between the exact magnetizations and KohnSham magnetizations in the $x^{-}, y$-, and $z$-directions for the first, second,

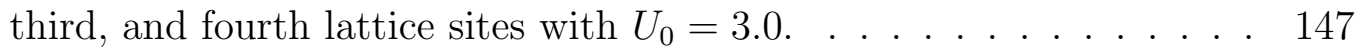

5.9 Magnetization differences between the exact magnetizations and KohnSham magnetizations in the $x$-, $y$-, and $z$-directions for the first, second, third, and fourth lattice sites with $U_{0}=9.0$. . . . . . . . . . . . . . 149

6.1 Structure and canonical orbitals of thiophene terminated benzothiadi-

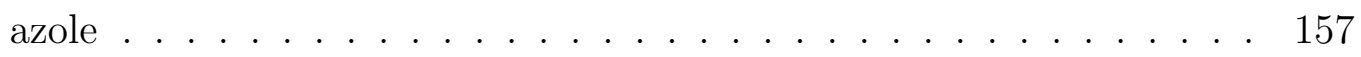




\begin{abstract}
Density-functional theory (DFT), in its various forms, has become a near ubiquitous form of theoretical research used to benchmark and prototype solutions to many finite and extended state system. This is largely because DFT can both capture the rich physics that is present in these electronic systems, while remaining computationally cost-effective and interpretable. However, DFT also has the requirement that the density functional being used to iteratively converge towards a solution must be accurate and correct. While on the surface such a stipulation seems benign, in practice the density functionals can be overwhelmingly complex and error can be introduced that comes from either the density functional that is chosen or the approximations used to make a system more calculationally tenable.

In this work, our focus is on the use of model systems to calculate and determine the usefulness and shortcomings of DFT. By simplifying the underlying system, while also retaining enough physical quantities from real systems, we can focus on how the approximations affect the outcomes that are produced. To begin, we show that charge-transfer dynamics can be described in unique and enlightening ways through the use of the particle-hole map (PHM). Using a one-dimensional, multi-well system, we effectively demonstrate how interesting electron dynamics can be uncovered by applying unitary transformations to the wavefunctions. By spatially localizing the electronic wavefunctions through the Foster-Boys method, which is analogous to Wannier localization in extended systems, the intermediating components of chargetransfer systems can be examined to determine their effect on the system-at-large. From the simple one-dimensional system, we could quickly infer real molecular sys-
\end{abstract}


tems that could potentially be examined using the same method to surmise the role charge-transfer intermediaries play in such systems as organic photovoltaics.

Beyond electron dynamics, the role of exchange-correlation (xc) scalar potentials and magnetic fields that are features of noncollinear spin Kohn-Sham (KS) and DFT was explored by comparing the exact Schrödinger solution to the KS and DFT approximations. By extending the Hubbard model to four sites, we can both solve the system exactly, while allowing for on-site and nearest-neighbor interactions. We were able to obtain benchmark solutions across a wide range of interaction strengths, determining that there are regimes where the xc magnetic fields play an increasingly larger role as the system becomes more correlated. In fact, there is a regime where the xc magnetic fields become larger than the external magnetic fields that are applied on the system. Through the model system, we could additionally compare the exact solutions against the approximated xc functionals and demonstrate that the weakly correlated regime can be adequately described by the xc functional approximations common to many real-systems.

Moving beyond steady state observations, we can also describe time-dependent electron dynamics through real-time TDDFT and use a model system to compare the time-evolution of the exact and KS solutions. By allowing the xc potentials to propagate in real time, we could explore the role the xc torques played during the evolution of a triangular lattice under an applied, time-varying magnetic field. Additionally, by controlling the spin-orbit coupling present in the small model system, we determined that the spin orbit coupling plays a substantial role in keeping the spins more closely aligned with the exact system. In part, this was due to the spin-orbit coupling serving as a time-varying magnetic field, which tended to be larger than 
the xc potentials that were also present. The trimer can also be quickly and easily expanded with the added spin-orbit coupling and compared to real model systems through computational physics software, such as Octopus. 


\section{Chapter 1}

\section{Introduction}

The purpose and direction of this thesis is to sufficiently describe and characterize how model systems can be used to observe the behavior of electrons in finite and extended systems. As such, this work seeks to compare well-established theoretical results and expound upon them to uncover areas in the many-body canon where current methods in Time-Dependent Density-Functional Theory (TDDFT) can be improved. Experimental results are still vitally important to any scientific endeavor so all results and theoretical conjectures rely on observable quantities that could be captured in experiment.

Model systems serve an important role, especially in the field of many-body physics because of their ability to be solved analytically, or at least with computational efficiency. Since density-functional theory (DFT) is an ab initio theory, we can easily and directly compare the results with those of the analytic, or numeric, solutions of the many-body Schrödinger Equation, if available. In this sense, having the model solution provides us with relevant physical insight and a basis of comparison that we 
would be unable to procure otherwise.

By removing the intricacies of a realistic system, we are able to focus on the component parts of a system. Using mathematically rigorous analogues for their real counterparts, we can tweak and control the influence of particular physical quantities. Finally, with that control at our disposal, we inform ourselves as to what physical processes are most responsible for the behavior we are witnessing in our model system. Then, because we have controlled and carefully selected our quantities, we can infer what physical behavior we would expect to take place when we reintroduce all of the competing complexities of a real system.

Importantly, we can directly compare observables for the exact solutions with the observables of our approximations. By doing this, we can immediately recover where theory falters and quickly correct how we might build a solution. Since we are performing research on physical materials, we focus on calculating and comparing physical observables.

To get to our model systems, we must first lay the ground work of how our systems can be created. In Chapter 2, we will discuss the theoretical framework on which this thesis is built, DFT, and introduce some model systems that have been used throughout physics with sometimes surprising accuracy. After a thorough review of what preceded and shaped this thesis, we will expand from ground-state DFT into the calculation of excited states and electron dynamics in Chapter 3. While in Chapter 3, we will introduce the localized particle-hole map and discuss intuitive ways to take a simple one-dimensional potential model and apply it within a molecular framework to explore charge transfer excitation processes.

Once we are thoroughly familiar with excitation dynamics and linear response 
TDDFT, we will maintain our theme of optimizing Kohn-Sham quantities in Chapter 4 by exploring exchange-correlation fields via a generalized Hubbard model framework. Because we choose to use a generalized Hubbard model, we can exactly diagonalize the many-body Schrödinger equation through a creative basis set selection. We will see that this exact solution allows us to compare our KS and exact potentials directly and identify regimes where the calculated exchange-correlation fields will have large effects.

In Chapter 5, we will again revisit the Hubbard model. Again, because we are afforded the luxury of solving the Hubbard model exactly, we can apply a small magnetic perturbation to our system and watch the magnetizations evolve in time. Now, rather than exploring the exchange-correlation effects with a static field, we can apply a time-dependent magnetic field and focus on the evolution of the magnetizations. Consistent with Chapter 4, we will see that there is a regime where the exchangecorrelation torques must be accounted for and can draw comparisons to more complex and realistic systems.

Following our final Hubbard model example, we will conclude this thesis with a conclusion in Chapter 6. In addition to wrapping up the primary accomplishments of this thesis, we will also postulate new directions for research. Unsurprisingly, much of the ideas and future directions are formulated around expanding upon the interesting foundations that were built by distilling the essence of the physics into more tractable and well-behaved model systems. 


\section{Chapter 2}

\section{Background and theory: the many-body problem}

One of the favorite anecdotes of my advisor is the idea that physicists learn quantum mechanics in kindergarten. More precisely, physicists learn how to solve quantum mechanical systems for electrons in kindergarten. The first system physicists learn is the particle-in-a-box, primarily because of the ability to be able to solve a system analytically, easily see quantization, and see how rich the physics can be in a simple system.

Importantly, when we approach a particle-in-a-box, we are dealing with a free electron, devoid of some of the physics that we readily see in daily life. In nature, there are not many cases where we deal with a well-defined space with a particle contained in it that is absent of any other external potentials.

One might naively think that there should be no way that such a simple idea could apply to real systems, given the large assumptions being made. But, there is 
some important physics belying that simple assumptions, being that electrons usually move quickly when compared to other objects. This is especially true when we look at electrons when compared to their fundamental brethren: protons and neutrons. Electrons, being lighter in mass than protons or neutrons, dart and dash in the welldefined energy levels around a nucleus because of the disparity in mass. This darting and dashing relative to the nucleons makes the nucleus appear as though they are stationary from the electron reference frame.

What turns out to be important and fundamental is the presence the nucleons have on the potentials the electrons feel. To that end, our assumption actually turns out to be far more reasonable than when initially postulated. By uncoupling the nucleons from the electrons, we are able to focus on the electron dynamics exclusively and explore the interesting phenomena that can be described in the following chapters.

\subsection{Separating the systems}

It is important to state that the difference in movement is more specifically a difference in the timescale of the associated movements of our two particle types. While the nucleons are still moving, vibrating and wandering around in space, just as electrons are, the timescale of their associated motions are much longer. We thus say that, compared to the electrons, the timescales of the nuclear motion is adiabatic and that their motion is essentially frozen-in-place, allowing us to decouple the nuclear and electronic equations of motion. 


\subsubsection{The Born-Oppenheimer Approximation}

Quickly after modern quantum mechanics was starting to establish itself [1-3], Born and Oppenheimer proposed an ingenious solution to deal with the complexities of physical systems [4]. As was previously mentioned, Born and Oppenheimer realized the nuclear and electronic parts of physical systems belonged to different timeresponse regimes. When in the electronic wavefunction's, $\Psi$, reference frame, the nuclear wavefunctions, $\Phi$, appear stationary.

In particular, extended state systems, such as common solids, where the nuclei create a more-or-less repetitive and stationary structure, are an obviously applicable case where the separation of the two degrees of freedom is intuitive. Less intuitively, but equally as applicable, are finite systems at relatively low temperatures. Away from hot temperatures, where nuclei begin to vibrate and translate with greater magnitude, the nuclei move slowly enough that electrons have ample amounts of time to react to any change in the nuclear coordinates. That leads to a steady-state approximation, where the movements of the nucleons are adiabatic, or sufficiently slowly varying in time that the system remains in an equilibrium state [5].

By using this assumption, we can decouple the electronic and nuclear Schrödinger equations, leaving us with a simplified manner in which to handle the energies of the system. After decoupling the equations, it is explicitly assumed that the energies of the system are simply the sum of the energy eigenstates of nuclear and electronic regimes,

$$
E_{\text {total }}=E_{\text {electronic }}+E_{\mathrm{n}, \text { vibrational }}+E_{\mathrm{n}, \text { rotational }} \text {. }
$$


Then, we recover the Schrödinger equation for the electronic system,

$$
\begin{aligned}
\left(\hat{H}_{e}\left(\mathbf{r}_{1}, \cdots, \mathbf{r}_{N}\right)+\sum_{i, \alpha} v_{e N}\left(\left|\mathbf{r}_{i}-\mathbf{R}_{\alpha}\right|\right)\right) \Psi_{s}^{\left\{\mathbf{R}_{\alpha}\right\}}\left(\mathbf{r}_{1}, \cdots, \mathbf{r}_{N}\right) & \\
& =E_{s}^{\left\{\mathbf{R}_{\alpha}\right\}} \Psi_{s}^{\left\{\mathbf{R}_{\alpha}\right\}}\left(\mathbf{r}_{1}, \cdots, \mathbf{r}_{N}\right) .
\end{aligned}
$$

Here, $\hat{H}_{e}$ is the electronic Hamiltonian and is dependent only on the position of the $N$ electrons of the system. The $v_{e N}$ term is the Coulombic repulsion from the $\alpha$-th nucleon on the $i$-th electron at positions $\mathbf{R}_{\alpha}$ and $\mathbf{r}_{i}$, respectively. Finally, $\Psi_{s}^{\left\{\mathbf{R}_{\alpha}\right\}}$ is the many-body electronic wavefunction that describes the $N$ electrons at positions $\mathbf{r}_{1}, \cdots, \mathbf{r}_{N}$ for the nuclear configuration with the nucleus at $\mathbf{R}_{\alpha}$, where $s$ specifies the complete set of quantum numbers for the electronic system.

We are also left with the nuclear Schrödinger equation,

$$
\left(\hat{H}_{N}\left(\left\{\mathbf{R}_{\alpha}\right\}\right)+E_{s}^{\left\{\mathbf{R}_{\alpha}\right\}}\right) \Phi_{\rho, s}\left(\left\{\mathbf{R}_{\alpha}\right\}\right)=\mathcal{E}_{\rho, s}^{t o t} \Phi_{\rho, s}\left(\left\{\mathbf{R}_{\alpha}\right\}\right) .
$$

Above, $\hat{H}_{N}$ represents the purely nuclear Hamiltonian, so $\Phi_{\rho, s}\left(\left\{\mathbf{R}_{\alpha}\right\}\right)$ represents the nuclear wavefunction with $\mathcal{E}_{\rho, s}^{\text {tot }}$ being the total electronic and nuclear energy of the system. It is important to note that the electron-nucleus Coulombic repulsion enters into the equation through the $E_{s}^{\left\{\mathbf{R}_{\alpha}\right\}}$ term, which is essentially the effective electronic energy term that determines the nuclear configuration and effective potential energy surface that the particles move across.

Now, we have two equations that define the two parts of our system. We see that the electron configuration enters as a parameter within the nuclear Schrödinger equation, giving us well-defined equations that will only break down as the dynamics 
of the nuclei begin to approach the time-scales on which the electrons operate. As long as the nuclear dynamics do not induce transitions between electronic states $\left(E_{s}^{\left\{\mathbf{R}_{\alpha}\right\}} \rightarrow\right.$

$E_{s}^{\prime\left\{\mathbf{R}_{\alpha}\right\}}$, then the decoupling will allow us to determine the nuclear dynamics, and thus the full many-body wavefunction, after we solve for the dynamics of the electrons. It turns out in many systems, it is the electronic dynamics that are the most difficult proposition to answer, as nuclei are fairly straightforward and simple to account for in the temperature and potential field regimes that we are interested in. As such, this thesis will focus on the methods used to calculate and determine the effect of these electronic dynamics.

\subsection{Introducing the problem}

From Eq. (2.2), we see that we can fully describe the electron dynamics of the system through the many-body wavefunction, $\Psi_{s}^{\left\{\mathbf{R}_{\alpha}\right\}}$. For clarity, let us define the Hamiltonian that enters in Eq. (2.2),

$$
\hat{H}=\sum_{i}^{N}\left(-\frac{\nabla_{i}^{2}}{2}+v_{\text {ext }}\left(\mathbf{r}_{i}\right)\right)+\frac{1}{2} \sum_{i \neq j}^{N} \frac{1}{\left|\mathbf{r}_{i}-\mathbf{r}_{j}\right|},
$$

where the electron-nucleus interaction, $v_{e N}\left(\left|\mathbf{r}_{i}-\mathbf{R}_{\alpha}\right|\right)$, has been replaced by a generalized $v_{\text {ext }}\left(\mathbf{r}_{i}\right)$. Here, and in the rest of this thesis, we will use atomic units ( $\hbar=e=a_{0}=m_{e}=1$ ). By defining our Hamiltonian in this way, we clearly and quickly recover the Schrödinger equation that would describe the electron dynamics of any system,

$$
\hat{H} \Psi_{s}\left(\mathbf{r}_{1}, \cdots, \mathbf{r}_{N}\right)=E_{s} \Psi_{s}\left(\mathbf{r}_{1}, \cdots, \mathbf{r}_{N}\right)
$$


We cannot jump up in elation yet, though, as we still have some major hurdles to cover in dealing with the many-body wavefunction, $\Psi_{s}$. Below, we will discuss the two main reasons why the wavefunction is difficult to deal with, and then introduce a big reason why we want to find a different way of describing our system.

1. The initial reason that we must be hesitant with our wavefunction is that we have many electrons to deal with in our system. While this thesis focuses on finite systems and models, we also want the features and physics discovered within the studies to be broadly applicable to larger systems. As such, we want to be able to accurately describe a system with any number of electrons, even solid state systems, where even small samples contain on the order of $\sim 10^{23}$ electrons. That number is exceptionally large on its own, but let's trim it back and go to a system that is closer to the model systems we will be discussing later.

Imagine we take only 100 electrons and throw them into a system that can be described by a single potential (this would be analagous to an atom with 100 electrons). We can perform a quick, back-of-the-envelope estimate on the amount of information that we will have to store to fully describe the wavefunction of this system. First, we describe the location of each electron in the system with its Cartesian coordinates. Using these coordinates, we have $3 \cdot N$ dimensional space complexity. Suppose we want to look at spin-dependent phenomena though? Now, we must take account of two additional coordinates, bringing our total tally up to $(3 \cdot 2 \cdot N$-coordinates. Quickly, we see that if we only worry about 3 entries for each electron of each spin, we have $3^{3 \cdot 2 \cdot N}$ entries that we must keep in memory in order to correctly calculate the full 
wavefunction. If we assume we can fully describe each entry by a single byte, we have $3^{600} \approx 10^{285}$ bytes to worry about. So, we could accurately describe this system if we were to have roughly four times the number of atoms in the known universe. While this initially seems extremely disturbing, in many cases, symmetry can help reduce the degrees of freedom (and thus dimensionality of the solution), but there is one huge caveat to this that is addressed in the second difficulty with dealing with the full many-body wavefunction.

2. The second, and more pressing, reason to be hesitant to simply stop with the formulation and calculation of the many-body wavefunction is that we must determine the electron-electron interactions and take them into account. Importantly, it is the interactions between electrons and nucleons and between the electrons in a system that determine and describe the physics taking place in them. Without accounting for the interactions, we would simply be unable to fully describe the physics in many realistic systems - at least without making some corrections that we will discuss in later chapters [6-8].

But, accounting for these interactions isn't free. Because of these correlated interactions, the Hamiltonian, $\hat{H}$ of our system cannot be separated into a summation of one-particle Hamiltonians, $\hat{h}_{i}$. This adds another layer of computational cost to the system, and that cost can be calculated as $\frac{1}{2} N(N-1)$ interactions that we must now account for (in a spin-independent scenario).

These two issues are paramount to our desire to develop another method to successfully computationally, and even analytically, characterize physical systems. Additionally, these two issues arise from simply trying to solve for the many-body wavefunction of the system of interest. One key caveat to solving for the many-body 
wavefunction is that the wavefunction itself is not an observable of the system. In this sense, we are directly identifying the key ingredient of our system, without being able to experimentally verify its accuracy.

The last statement is perhaps more provactive than it ought be. Experimentalists can use other observables that are derived from the wavefunction to determine whether their results follow the solutions of the many-body Schrödinger equation. So, in truth, what we are actually in pursuit of is not the wavefunction itself, but rather the observable quantities that can be derived from it.

\subsubsection{Observable quantities}

In any exploration of a physical system, what we pursue - and what particularly matters - is a verifiable signature that can be used to justify the underlying description

of that system. Essentially, we are searching for quantities that are illustrative of the wavefunction itself, while being both easier to handle computationally and physically verifiable via experimental methods. In this thesis, there are a few key observables and quantities that will be necessary to form the foundation of all the subsequent work.

\section{Total energy}

The first key quantity that we need to introduce is the total energy. We can easily capture the total energy by rearranging Eq. (2.5) and solving with respect to $E_{s}$. After rearranging, we get

$$
E_{s}=\int d^{3} r_{1} \cdots d^{3} r_{N} \Psi_{s}^{*}\left(\mathbf{r}_{1}, \cdots, \mathbf{r}_{N}\right) \hat{H} \Psi_{s}\left(\mathbf{r}_{1}, \cdots, \mathbf{r}_{N}\right)
$$


We can see that $E_{s}$ is now defined as a functional of the wavefunction.

The minimum value of $E_{s}$, the ground state - $E_{0}$, is vitally important to many different areas of physics research. The reason for this is that it is the lowest possible energy state that a material or molecule may be in. It usually constitutes the reference state of system upon which we can explore the influences of various kinds of perturbations. Also, in many cases, the ground state is usually the occupied state, even at finite temperatures near room temperature. While we will not be dealing with any finite-temperature effects here, much of the physics taking place in condensed matter research can be captured with low-temperature calculations.

\section{Density}

Even simpler perhaps than the total energy is the electronic density of the system, $n(\mathbf{r})$. While the wavefunction contains all of the information needed to fully characterize a system, we can quickly simplify the wavefunction while retaining a large amount of knowledge about the system that we can leverage in the future. We begin by defining the electronic density as

$$
n(\mathbf{r})=N \int d^{3} r_{2} \cdots d^{3} r_{N} \Psi_{s}^{*}\left(\mathbf{r}_{1}, \mathbf{r}_{2}, \cdots, \mathbf{r}_{N}\right) \Psi_{s}\left(\mathbf{r}_{1}, \mathbf{r}_{2}, \cdots, \mathbf{r}_{N}\right)
$$

which we can decipher as $n(\mathbf{r})$ being the number of electrons per unit volume. This quantity is directly related to the probabililty amplitude of finding an electron at $\mathbf{r}$. Additionally, the density reduces the number of variables we must concern ourselves with from $3 N$ to just 3 . We will see in the next section that this plays an incredibly important role in being able to handle calculations that can be performed on real 
systems and larger numbers of electrons. Finally, we will see that this quantity can be nicely expanded when we enter into a spin-dependent basis, and captures the magentic dyanmics of the system.

\subsection{Recasting the problem}

As mentioned above, working with the full many-body wavefunction of the system is unwieldy and unmanageable for even small systems. To even begin to approach real systems, we must reformulate our problem to solve for quantities other than the wavefunction. To accomplish this, we will use the two observables we discussed above and construct an effective theory in terms of them. Remember, our observables have reduced the storage cost of our many-body wavefunction and that storage efficiency will give us leverage as we scale up to more-and-more electrons.

\subsubsection{Variational method}

Above we described the total energy of the system and specifically focused on one key quantity, $E_{0}$, which corresponds to the energy of the ground state of the system. That is, $E_{0}$ is the lowest possible energy state, and thus eigenstate, that our system can be in. According to the Rayleight-Ritz variational principle, $E_{0}$ is obtained as the variational minimum of the functional

$$
E_{0}[\Phi]=\int \Phi^{*}\left(\mathbf{r}_{1}, \cdots, \mathbf{r}_{N}\right) \hat{H} \Phi\left(\mathbf{r}_{1}, \cdots, \mathbf{r}_{N}\right)
$$


In other words, we have defined $E_{0}$ in a way that any other function we choose for $\Phi$ that satisfies the boundary conditions of our system cannot be less than $E_{0}$. Put another way, if we expand our function $\Phi$ into another basis, such as: $|\psi\rangle=$ $\sum_{n} c_{n}\left|\phi_{n}\right\rangle$, we have set a lower bound on our system which we cannot go below. This pricinple is the key idea for using such method as configuration interaction or variational Monte Carlo. Advantageously, it also sets a basis with which we can expand into and finally begin broaching the subject of Hartree-Fock (HF), KohnSham (KS), and density-functional theory (DFT), but before we get there, we visit one more idea.

\subsubsection{Functionals and reformulating the many-body Schrödinger equation}

Recall that our end goal is not to simply calculate the many-body wavefunction. While that would allow us to completely determine our system, the difficulty lies in actually calculating that quantity. What we might do instead is recast the problem: where instead of worrying about the wavefunction, we are concerned with how the observables change for systems with differing potentials. We can hypothesize that the electronic density might depend only on the underlying external potential that is present and influencing the electronic behavior. So, what we would like to be able to show is that a specific external potential uniquely determines the density of the system. That is, we want to know that there is a one-to-one mapping from an external potential to a density.

First, we must ask what are the potentials and expectation values we are dealing with. After all, we must be able to correctly characterize our system before we go 
about reinterpreting the Schrödinger equation. We see that the energy expectation value is just $\langle\Psi|\hat{H}| \Psi\rangle$, which from Eq. (2.4), can be broken down as

$$
\langle\Psi|\hat{H}| \Psi\rangle=\left\langle\Psi\left|\sum_{i}^{N} v_{\text {ext }}\left(\mathbf{r}_{i}\right)+\hat{T}+\hat{V}_{e e}\right| \Psi\right\rangle
$$

where $\hat{V}_{e e}=\frac{1}{2} \sum_{i \neq j}^{N} \frac{1}{\left|\mathbf{r}_{i}-\mathbf{r}_{j}\right|}$ and $\hat{T}$ is the kinetic energy operator.

If we apply our expectation values on the first term, we recover

$$
\left\langle\Psi\left|\sum_{i}^{N} v_{\text {ext }}\left(\mathbf{r}_{i}\right)\right| \Psi\right\rangle=N\left\langle\Psi\left|v_{\text {ext }}(r)\right| \Psi\right\rangle=\int v_{\text {ext }}(\mathbf{r}) n(\mathbf{r}) d \mathbf{r}
$$

We also remember that the ground state of the system, according to Eq. (2.8), is the state corresponding to the minimization of the energy, $E_{0}$. But, recall, we don't want to use the wavefunction because of its complexity, so what if we reformulate to minimize our energy with respect to an observable? How could we solve this system for that observable, but still accurately capture the ground state? We would need to find

$$
E_{\mathrm{GS}}=\min _{n}\left\{\int v_{\mathrm{ext}}(\mathbf{r}) n(\mathbf{r}) d \mathbf{r}+F_{\mathrm{HK}}[n]\right\},
$$

where $F_{\mathrm{HK}}[n]$ is a functional of the density that describes the electron-electron interactions and kinetic energy. If we fully expose $F$, we find

$$
F_{\mathrm{HK}}[n]=\left\langle\psi_{\mathrm{HK}}\left|\hat{T}+\hat{V}_{e e}\right| \psi_{\mathrm{HK}}\right\rangle
$$

While, Hohenberg and Kohn originally showed that the minimization only holds for nondegenerate ground states, Levy and Lieb later generalized this idea through constrained search [9-11]. Notably, $\psi_{\mathrm{HK}}$ can be the many-body wavefunction, but as 
defined, it can also be any wavefunction whose density is identical to the density that minimizes Eq. (2.11).

\subsubsection{Noninteracting particles}

Previously, it was mentioned that we lose some of the information contained within the wavefunction if we make some assumptions about the system we are working with. However, we can recapture it in a way that preserves most of the physics while allowing us to more easily solve the system of equations before us. To do this, we move away from the interacting, many-body framework and instead make a simple ansatz that our observables can be described by noninteracting single-particle

orbitals. While our assumption unrealistic, we will see that our substitution gives us more tractable equations that allow us to leverage computational tools to solve realistic physical systems.

\section{Effective electronic interaction and Hartree approximation}

To begin, we assume that we can approximate the electronic interactions through an effective single particle potential that is caused only by the Coulombic term. Our electron-electron potential term, $V_{e e}$, can now be written as,

$$
V_{e e}=\int d \mathbf{r}^{\prime} \frac{n\left(\mathbf{r}^{\prime}\right)-n_{j}\left(\mathbf{r}^{\prime}\right)}{\left|\mathbf{r}-\mathbf{r}^{\prime}\right|}
$$

where the first term is the Hartree Potential, while the second term is the selfinteraction correction, preventing an electron from feeling a Coulombic repulsion to 
itself. Here, we define our densities as

$$
n(\mathbf{r})=\sum_{j}^{N} n_{j}(\mathbf{r})=\sum_{j}^{N}\left|\phi_{j}(\mathbf{r})\right|^{2}
$$

It is also clear that our electron-electron term depends on the effective electron density of the $j$-th state. We shall see that this state dependence is a defining feature of Hartree-Fock theory and one key differentiator from Kohn-Sham (and DensityFunctional) theory. To derive Eq. (2.13), we assume our $N$-particle wavefunctions are a simple product of the single particle orbitals,

$$
\Psi\left(\mathbf{r}_{1}, \cdots, \mathbf{r}_{N}\right)=\phi_{1}\left(\mathbf{r}_{1}\right) \cdots \phi_{N}\left(\mathbf{r}_{N}\right)
$$

Now, let us revisit our Hamiltonian in Eq. (2.9) and derive our Hartree-Fock equations from the variational principle. Instead of minimizing our Hamiltonian with respect to the many-body Schrödinger wavefunctions, we will minimize with respect to our noninteracting electronic wavefunctions. Using Eq. (2.15), we can carry out the variation of the expectation of the many-body Hamiltonian, Eq. (2.9). Through some straighforward calculus and algebra, one captures the Hartree equation,

$$
\left[-\frac{\nabla^{2}}{2}+v_{\mathrm{ext}}(\mathbf{r})+\int d \mathbf{r}^{\prime} \frac{n\left(\mathbf{r}^{\prime}\right)-n_{j}\left(\mathbf{r}^{\prime}\right)}{\left|\mathbf{r}-\mathbf{r}^{\prime}\right|}\right] \phi_{i}^{H}(\mathbf{r})=\varepsilon_{i} \phi_{i}^{H}(\mathbf{r}) .
$$

\section{The Slater determinant and Hartree-Fock equation}

While Eq. (2.16) is very close to where we want to go, there is an incredibly important feature that is missed in Eq. (2.16) due to our many-body wavefunction approxima- 
tion in Eq. (2.15). It's clear on inspection that the Hartree Product expansion of our wavefunctions in Eq. (2.15) is symmetric. Because we are dealing with fermions, we necessarily need antisymmetric wavefunctions. To capture the antisymmetric nature of fermionic wavefunctions, we can instead approximate our many-body wavefunction as a Slater determinant of our single particle orbitals, [12]

$$
\Psi\left(\mathbf{r}_{1}, \cdots, \mathbf{r}_{N}\right)=\frac{1}{\sqrt{N !}} \operatorname{det}\left|\begin{array}{ccc}
\phi_{1}\left(\mathbf{r}_{1}\right) & \cdots & \phi_{1}\left(\mathbf{r}_{N}\right) \\
\vdots & \ddots & \vdots \\
\phi_{N}\left(\mathbf{r}_{1}\right) & \cdots & \phi_{N}\left(\mathbf{r}_{N}\right)
\end{array}\right|
$$

Once again, we can plug these new wavefunction approximations into Eq. (2.9) and carry out another variation of the expectation value of the Hamiltonian. This time, due to our new single particle expansion that preserves the antisymmetry of fermionic wavefunctions, we capture the Hartree-Fock Equation:

$$
\left[-\frac{\nabla^{2}}{2}+v_{\mathrm{ext}}(\mathbf{r})+\int d \mathbf{r}^{\prime} \frac{n\left(\mathbf{r}^{\prime}\right)-n_{j}\left(\mathbf{r}^{\prime}\right)}{\left|\mathbf{r}-\mathbf{r}^{\prime}\right|}\right] \phi_{j}(\mathbf{r})-\sum_{i}^{N} \int d \mathbf{r}^{\prime} \frac{\phi_{i}^{*}\left(\mathbf{r}^{\prime}\right) \phi_{i}(\mathbf{r})}{\left|\mathbf{r}-\mathbf{r}^{\prime}\right|} \phi_{j}\left(\mathbf{r}^{\prime}\right)=\varepsilon_{j} \phi_{j}(\mathbf{r})
$$

Here, we note that the HF equation features a nonlocal potential - the final term on the left-hand side of Eq. (2.18) - which is mathematically seen as an integral over $\mathbf{r}^{\prime}$. However, this term can also be interpretted as an operator acting on the single particle orbtial at position $\mathbf{r}, \phi_{j}(\mathbf{r})$, such that the result is dependent on the $\mathbf{r}^{\prime}$ integration. One important note is that the Hartree potential, the last term on the left-hand side of Eq. (2.16), is only multiplied onto the single particle orbital $\phi_{j}(\mathbf{r})$. 


\section{Kohn-Sham Equations}

While Hohenberg and Kohn built upon Hartree and Fock's idea that one can use simpler, noninteracting particles to recreate the exact many-body density, the HF computational method was ill-equipped to efficiently and accurately perform computations on real systems. In HF, the presence of the nonlocal potential we discussed above, in Eq. (2.18), is a hefty computational challenge to solving the system of equations. So, what are we to do? What we can do is redefine our system, such that we have an all encompassing effective potential, $v_{\mathrm{KS}}(\mathbf{r})$ - known as the Kohn-Sham potential, that accounts for both our external and electron-electron potentials [13],

$$
\left(-\frac{1}{2} \nabla^{2}+v_{\mathrm{KS}}(\mathbf{r})\right) \varphi_{i}(\mathbf{r})=\varepsilon_{i} \varphi_{i}(\mathbf{r}) .
$$

After defining the effective potential for our system, the density is given by

$$
n(\mathbf{r})=\sum_{i}^{N}\left|\varphi_{i}(\mathbf{r})\right|^{2},
$$

which is clearly just the density of the wavefunctions that are the eigenfunctions of our effective potential system. Regardless of our choice of $\varphi(\mathbf{r})$, we will be able to iteratively proceed towards our exact density, guaranteed by the variational principle to have a minimum at our exact ground state density. It is difficult to overstate the idea that we've created a solution to the many-body problem by using noninteracting particles, yet in a scenario where the exact functional is known, we can produce the exact ground state density with a corresponding noninteracting potential. In Figure 2.1, we clearly see that if we know our solution is the ground state of our system, we recover the exact density (albeit from our KS potential rather than the exact 


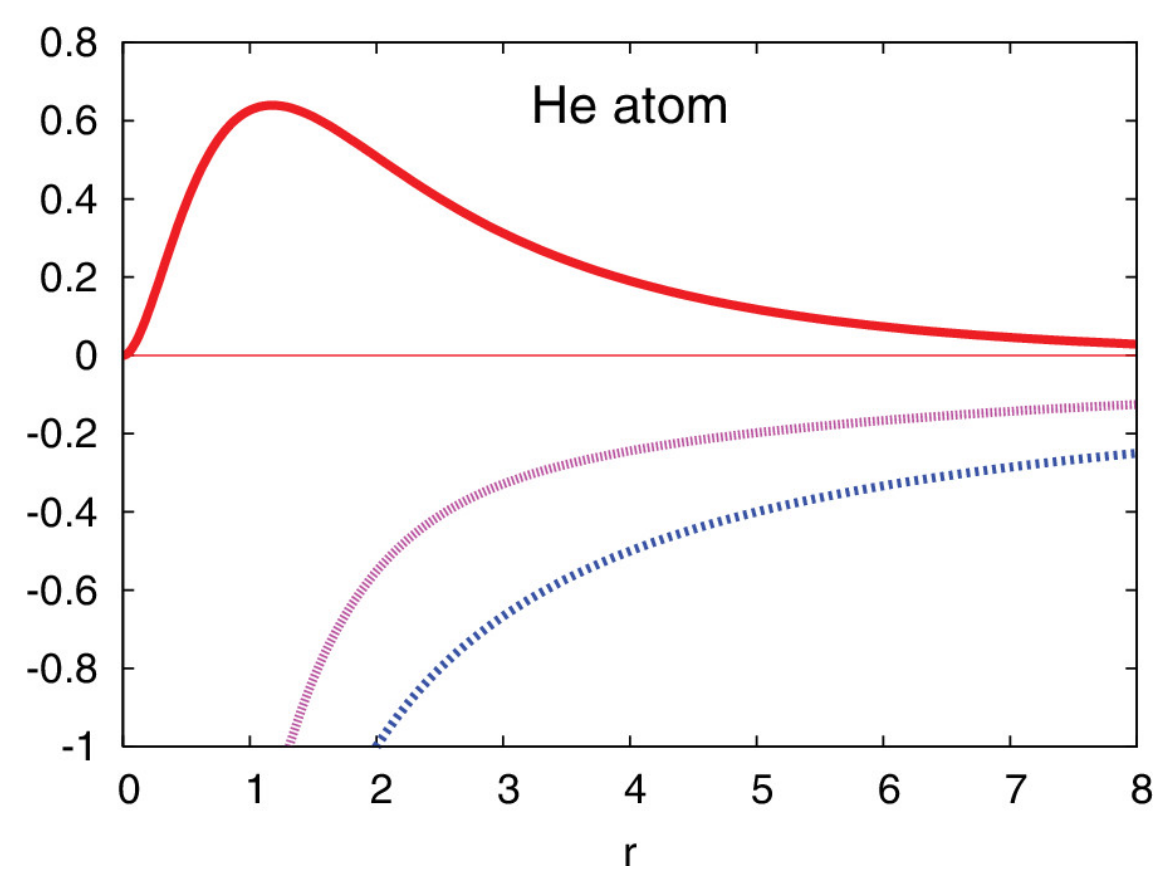

Figure 2.1: Exact radial density (red) for Helium. The calculated Kohn-Sham potential (purple) for two non-interacting electrons produces the same density as the exact potential (blue). Adapted from [14]

potential).

The total energy is then defined as a functional of the density,

$$
E[n]=\hat{T}[n]+\int d \mathbf{r} v_{\text {ext }}(\mathbf{r}) n(\mathbf{r})+E_{\mathrm{H}}[n]+E_{\mathrm{xc}}[n] .
$$

Here, the kinetic term is defined as in the Hartree-Fock Eq. (2.18) and $E_{\mathrm{H}}$ is the Hartree energy as before

$$
E_{\mathrm{H}}[n]=\frac{1}{2} \int d \mathbf{r} \int d \mathbf{r}^{\prime} \frac{n(\mathbf{r}) n\left(\mathbf{r}^{\prime}\right)}{\left|\mathbf{r}-\mathbf{r}^{\prime}\right|}
$$

The difference is that we now have a collected energy term, $E_{\mathrm{xc}}$ that contains the exchange energy from the Hartree-Fock equation. This $E_{\mathrm{xc}}$ is essentially our catch all 
that accounts for the electron exchange potential, but also takes into account some of the Coulombic repulsion that lost by simply approximating that repulsion over the electronic densities rather than through the exact electron locations.

To some extent, this feels almost sacriligious, however, we have done nothing to lose generality. On the contrary, we have actually improved our computational outlook, as now, we don't have to worry about the nonlocal exchange potential. To that end, when we match terms from Eq. (2.21) and vary them with respect to the set of orbitals subject to the constraint that those orbitals must reproduce the exact ground state density, we see that

$$
v_{\mathrm{KS}}(\mathbf{r})=v_{\mathrm{ext}}(\mathbf{r})+\int \frac{n\left(\mathbf{r}^{\prime}\right)}{\left|\mathbf{r}-\mathbf{r}^{\prime}\right|} d \mathbf{r}^{\prime}+\frac{\delta E_{\mathrm{xc}}[n]}{\delta n(\mathbf{r})}
$$

Here, we can see that the second term is just the Hartree potential, $v_{\mathrm{H}}$, while we define the final term as the exchange-correlation potential $v_{\mathrm{xc}}$. Upon inspection of $v_{\mathrm{H}}$ in Eq. (2.23), we see that the Kohn-Sham Hartree potential is defined identically to Eq. (2.13) from the Hartree-Fock approximation. The difference between Eqs. (2.23) and (2.13) comes from the definition of the wavefunctions, where $v_{\mathrm{H}}$ from Eq. (2.23) uses the KS wavefunctions, $\varphi_{\mathrm{KS}}$, and corresponding $n_{\mathrm{KS}}$.

By creating this definition of the exchange-correlation potential, we have simplified the computational task of solving for the noninteracting wavefunctions, $\varphi_{i}$ which exactly recreate our many-body density. Our sacrifice in rewriting our equations in this way is that we lose some clarity into the exchange and correlation terms that are collected within $v_{\text {xc }}$. While the exact KS equations are self-interaction free, it is not explicitly obvious how the self-interaction exactly cancels in the KS formalism. By contrast, in HF there is a clear and explicit cancellation of the self-interaction error. 


\subsection{Functional approximations and time-dependence}

Calculating the time-independent steady state of an electronic system is extremely important. By altering the Schrödinger equation and turning it into a system of functional equations with noninteracting wavefunctions, we have allowed for the computation of the energetics and electronic observables from first principle methods.

From this point, there are many branches that can be taken out into the world of DFT. One could stay in the steady state and calculate the ground state properties for solids and extended systems. Once the ground state is solved self-consistently after the proper choice of functional, a theorist can explore numerous avenues such as calculating the dielectric constant or the electronic response function [15]. Importantly, these quantities can be verified through the use of optical absorption spectroscopy or electron energy loss spectroscopy. By transforming those equations into reciprocal space, a theorist would be able to calculate the band structures and other excitation

effects for many materials $[16,17]$. While extended systems are worthwhile to explore and there are numerous parallels to finite systems, we will not spend time exploring this rich ecosystem.

Instead, we will focus on finite systems, and more specifically model systems, to determine what role the choice of basis and exchange-correlation approximation plays on the calculations of those systems. In finite systems, we can also calculate energetics, force fields, and geometries using the principles mentioned above, and explore the effects of varying the functional approximations that are used for $v_{\mathrm{xc}}$ in Eq. (2.23). Unsurprisingly, the choice of functional plays a large role in the ability of DFT to accurately calculate energy levels and densities. 


\subsubsection{The exchange-correlation potential}

We have defined the xc potential as the functional derivative of some exchangecorrelation energy. What is that energy? Previously, we waved our hands a bit and said it is simply the energy difference between our exact system and the system that is satisfied by noninteracting wavefunctions/particles. But, there is more to it, and for that we can glance back at Hartree-Fock for an explanation.

We saw when we derived the HF equation that when we chose to have noninteracting wavefunctions, there was a term that was the manifestation of the Pauli exclusion principle. That is to say, the exchange term is the repulsive term that we know electrons physically display: if they have the same spin state, they cannot occupy the same energy state. But, what is the correlation term and how do we reconcile it?

Recall that our Hartree term is actually the electronic density-density Coulombic term. We need the Coulombic term to obviously maintain the electrostatic forces that are present in our system, but by losing the exact location of the electron and using the density in its stead, we are using some amount of approximation on the Coulombic repulsion. To correct this, we must take into account that the electronic density is being used to calculate the elecrostatic repulsion of the particles. This correction is what defines the correlation term in our xc potential. Rather than defining the exchange outright (immediately), we group the terms together, knowing that the exchange term can be defined in a similar fashion to $\mathrm{HF}$, while the correlation must be determined a posteriori. Once our density is calculated and known, we know that the correlation must account for the difference between our exact total energy and 
our calculated $E_{\mathrm{KS}}$. Thus, the correlation energy can be defined as

$$
E_{\mathrm{c}}=E_{\text {exact }}-E_{\mathrm{HF}} .
$$

Defining our exchange and correlation energies as separable quantities allows us to devise treatment of the terms separately. In addition to being able to separate the exchange and correlation potentials, we notice that there must be some difference in our kinetic energy terms that comes from our switch to noninteracting particles. As we've done before, it is easy to collect this term inside of our correlation potential, as we've already defined it to be the energy difference between our exact solution and the HF, noninteracting system. Using this definition, our correlation energy must depend on potential and kinetic terms. Gathering the kinetic term within the correlation potential, $v_{\mathrm{c}}$, we define the correlation potential as,

$$
v_{\mathrm{c}}=v_{\mathrm{ext}}-v_{\mathrm{H}}-v_{\mathrm{x}} .
$$

The separability of exchange and correlation means we can derive functionals that account for each term independently and provides us a segue into the world of functionals.

\subsubsection{The world of functionals}

Up to this point in our story, there has been no true approximation. Indeed, we have altered our exact wavefunction such that we can use more computationally efficient observables such as the density. We also used a more advantageous definition of our particles so we could remove the many electronic interaction terms that would also 
add computationally inefficient terms. Those adjustments could be captured directly within our exchange-correlation term, leading to exact solutions.

The exact solution is dependent on an exact exchange-correlation functional, though. Unfortunately, the xc functional that broadly applies to all materials is too complex to solve for and hasn't been determined analytically (yet...), so we must approach our systems with an approximate functional that will satisfactorily solve for the energetics of our chosen systems. While there is no single approximation that accurately captures the physics of all systems, we have many methods and approximations that work well in specific cases. Here, we will briefly explore the breadth of different functional flavors and types.

\section{Functional flavors}

Within the world of functionals there are two primary flavors of approximations. One can be thought of as derived from first principles, satisfying certain known exact conditions of the exchange-correlation functional. The other can be thought of as using experimental data as the basis from which parameter tuning and fitting form the basis of the approximation.

- Orbital dependent and non-empirical functionals The orbital dependent functionals are the primary focus of this research as they allow for broad application as long as the KS wavefunctions can be easily maintained and calcultated. They have the additional flexibilty of being directly expandable by moving into noncollinear spin-dependent systems without any loss of generalization. It is primarily for this reason in Chapters 4 and 5 that we use the approximation to exact exchange known as the Slater potential. By sidestepping the parameter 
fitting, we can broadly take account of how orbital based measures perform while also inspecting the effects that correlation has on our approximations. One important area where the orbital dependent functionals tend to underperform are in energy calculations due to noninteracting electron approximation. This underperformance is across many extended, atomic, and molecular system calculations.

- Empirical functionals Through inspection of common elements/systems we could fit functionals that most accurately characterize those systems. Obviously, the generalizability of these empirical functionals is not nearly as broad as orbital-dependent functionals. That said, empirical functionals tend to have lower errors for the energetics that they are trying to describe when compared to their orbital dependent counterparts. If the empirical parameters and quantities are well chosen, such that they accurately fit a broad base of systems, they can have fairly broad and accepted applicability. Unfortunately, empirical functionals tend to be unsystematic in their error as they are created to succeed in a defined environment and are thus difficult to interpret generally across systems where they may behave erratically.

In this thesis we will focus entirely on the orbital dependent flavors of functional approximations as they tend to have the broadest applicability. Additionally, their errors can be examined through inspection of the calculated observables and compare them to the exact solutions to derive systematic errors due to our functional approximations. 


\section{Functional types}

We touched upon the different flavors of functionals that are commonly used in DFT calculations, but there are overarching types of functionals that are grouped by the method used to create the approximation. Some types are very simple, relying on the density at a single point in space or assuming that the density is uniformly distributed across the system, while other methods rely on the density gradients in an effort to more accurately capture the density while sacrificing computational efficiency. Generally, there are four basic groups and they grow in complexity and should reduce the error as the complexity grows.

- The first and most basic type are those of the local density approximations (LDA). The LDA functionals generally either rely on the observed density at a single point in space and assume a slowly varying density (to account for this change) or they assume a uniformly distributed density (such as the uniform electron gas). While simplistic, LDA functionals can capture the electron dynamics and electrostatics of the system. However, LDA functionals also tend to be poor estimators of energy levels and energy gaps, leaving their most useful properties their computational and memory efficiencies.

- The next step up in complexity are the generalized gradient approximation (GGA) functionals. In a GGA functional, rather than assuming uniform distribution of the density, or slowly differing enough to be calculable via localized operations, the functional also uses the gradient of the density. Obviously, by including the gradient, the change of the density is properly accounted for and more accurately characterizes the density space of the system. A more accurate 
account of the density space leads to more accurate observables and GGAs do a better job of calculating the energetics of extended systems. Some of the most widely used functionals, PBE or BLYP for example, are GGA functionals and they are a popular workhorse throughout the DFT community [18-20]. The trade-off for accuracy, though, is the requirement that the gradient must be calculated and stored for each point in the calculation space, adding computational heft and complexity. Using GGAs for spin-independent or spin-polarized systems is still easy to attain in practice, but the added complexity of noncollinear spin systems, where the single wavefunction is now a spinor, a length two spin vector, makes GGAs much more difficult calculate (or construct) for real-time and time-dependent propagations.

- Going one step beyond GGAs brings us to the land of meta-GGAs. As with normal GGAs, meta-GGAs rely on both the density and the density gradient at each point in the functional space. However, meta-GGAs also depend on the Laplacian of the electron and/or kinetic energy density. As one can imagine, the additional computed quantity allows for more accurate calculations, but the problems with storage and computation from a single gradient is exacerbated due to a second set of gradients that must be stored for the entire functional space. One more difficulty posed by meta-GGAs are the difficulties posed in calculating the functional derivative of the kinetic energy density.

- Finally, we reach the most widely applied type of functional approximations: hybrids. Hybrids, generally utilize the separation of the exchange and correlation functions to apply different techniques on each term. To reduce the computational heft of calculating the nonlocal exchange term, some amount of the 
exchange term is generally approximated with a local density approximation, while the rest is calculated fully nonlocally. The percentage of the exchange that is still fully nonlocally calculated is still a large amount of computational heft, regardless. Still, hybrid functionals give very good results when used on systems they were designed for and one of the most popular functionals, B3LYP (a favorite among organic chemists), is a hybrid functional [19-21].

These functional types are not all encompassing and with the popularity of DFT, many of the listed functionals will probably be obsolete by the time we have finished typing this sentence. In addition to the overarching types of functionals, there is much research in long-range corrections to the functionals above to better characterize electron characteristics in large $\mathbf{r}$ regions and charge transfer systems that will be discussed in chapter 3. In our work, we focus primarily on the exact exchange (EXX) functional, which will be discussed in more depth in chapter 4, along with the KLI approximation to the EXX functional. Finally, we will briefly discuss and cover the STLS method, which includes correlation effects through the unoccupied orbitals, and creates a self-consistent way to calculate $E_{\mathrm{xc}}$.

\subsubsection{Dynamic systems and time dependence}

Previously, we discussed that Hartree and Fock presented a variational method to solve for the exact ground state density of any system that was later expanded and further developed by Hohenberg and Kohn. Once placed within a self-consistent framework by Kohn and Sham, we reached a point where large, many-electron systems could be calculated straightforwardly and within hours on almost any laptop computer. Having a basis for the ground state and a variational framework that 
shows we can theoretically attain exact solutions, we turn our gaze towards timedependence.

While a large amount of physics can be calculated and explored in just the ground state and static situations, the world of electron dynamics is even richer. In today's modern world, we interact daily with electronics, primarily made up of inorganic semiconductors. Such materials have extremely advantageous properties that allow for the manipulation of the band gaps through doping, allowing us to use them as transistors, storage devices, and photovoltaics. So, these different materials have characteristics that we have become accustomed to taking advantage of in this modern world, but all of those properties can be calculated and refined using time-dependent density-functional theory (TDDFT) techniques.

To properly characterize these systems and be confident in our results though, we must ensure our ground state formalism is generalizable to characterize electronic dynamics and time dependence. In order to do this, we must approach the timedependent case much in the same way as the time-independent (re: ground state) case. Our problem will be to determine the following: given a time-dependent density, $n(\mathbf{r}, t)$, show that there is a one-to-one correspondence to a time-dependent external potential, $v_{\text {ext }}(\mathbf{r}, t)$, for a given initial state.

\section{The Runge-Gross Theorem}

We begin in largely the same place that we began for the HK theorem, which is useful since we are making an analogous argument to that steady state situation. Since we are in time-dependent quantum regime, we are governed by the time-dependent 
Schrödinger equation,

$$
\hat{H}(t) \Psi(t)=i \frac{\mathrm{d} \Psi(t)}{\mathrm{d} t}
$$

where we have begun in some prepared eigenstate of the Hamiltonian, $\hat{H}$ (i.e. $\Psi(t=0)$ is known). Here, $\hat{H}$ is defined in the same was as Eq. (2.9), where $\hat{V}_{\text {ext }}$ is now defined

$$
\hat{V}_{\mathrm{ext}}=\sum_{i}^{N} v_{\mathrm{ext}}\left(\mathbf{r}_{i}, t\right)
$$

so our external potential is now time varying and changes according to the system being investigated.

To prove that our time-dependent potential maps one-to-one to a time-dependent density, we consider two external potentials, $v_{\text {ext }}(\mathbf{r}, t)$ and $v_{\text {ext }}^{\prime}(\mathbf{r}, t)$, that differ by more than an additive constant. Instead of using the density of the system, we use the current density, defined as

$$
\mathbf{j}(\mathbf{r}, t)=N \int d \mathbf{r}_{2} \cdots \int d \mathbf{r}_{N} \operatorname{Im}\left\{\Psi\left(\mathbf{r}_{1}, \cdots, \mathbf{r}_{N} ; t\right) \nabla \Psi^{*}\left(\mathbf{r}_{1}, \cdots, \mathbf{r}_{N} ; t\right)\right\}
$$

where $\operatorname{Im}\{f\}$ denotes the imaginary part of $f$. Using the continuity equation from the Schrödinger equation

$$
\frac{\partial n(\mathbf{r}, t)}{\partial t}=-\nabla \cdot \mathbf{j}(\mathbf{r}, t)
$$

we can examine the equation of motion of the difference between two current densities, $\mathbf{j}(\mathbf{r}, t)$ and $\mathbf{j}^{\prime}(\mathbf{r}, t)$.

After working through the equation of motion, we discover a $\nabla\left\{v_{\text {ext }}(\mathbf{r}, 0)-v_{\text {ext }}^{\prime}(\mathbf{r}, 0)\right\}$ term. We can clearly see, then, that if our external potentials that define our current densities differ by more than an additive constant, the first order derivative of the 
currents must differ as well. Expanding the differentiation of $\mathbf{j}(\mathbf{r}, t)$ to the $k$-th term and evaluating at $t=0$, we find

$$
\frac{\partial^{k+1}}{\partial t^{k+1}}\left\{\mathbf{j}(\mathbf{r}, t)-\mathbf{j}^{\prime}(\mathbf{r}, t)\right\}_{t=0}=-n_{0}(\mathbf{r}) \nabla \frac{\partial^{k}}{\partial t^{k}}\left\{v_{\text {ext }}(\mathbf{r}, t)-v_{\text {ext }}^{\prime}(\mathbf{r}, t)\right\}_{t=0}
$$

As long as the potentials, $v$ and $v^{\prime}$, differ by more than an additive constant and can be expanded as a Taylor series about $t=0$, there must be a finite $k$ where the right hand side of Eq. (2.30) is nonzero. That obviously implies that $\mathbf{j}(\mathbf{r}, t) \neq \mathbf{j}^{\prime}(\mathbf{r}, t)$ which shows that a current density is uniquely determined by a given external potential.

All that is left is to show that the currents are unique to a given density, $n(\mathbf{r}, t)$. This follows by applying the gradient to Eq. (2.30). I will leave the final pieces of that proof to Runge and Gross, but suffice it to say, the current densities are uniquely determined by our electronic density, giving us a one-to-one map from time-dependent potentials to time-dependent densities $[22,23]$. This allows us to easily generalize the KS equations to a time-dependent system as

$$
i \frac{\partial \varphi_{i}(\mathbf{r}, t)}{\partial t}=\left[-\frac{\nabla^{2}}{2}+v_{\mathrm{KS}}[n](\mathbf{r}, t)\right] \varphi_{i}(\mathbf{r}, t)
$$

Here, we have a differential equation that is of the exact same form as the Schrödinger equation, and we can propagate our states forward in time the exact same way. We now have shown that we can time evolve our KS orbitals, knowing that we have a direct one-to-one relationship between the time-evolving external potential and timeevolving wavefunctions. Again, we have shown that our formalism is mathematically well-defined for our potential experiments, but we again have computational hurdles that must be overcome. In particular, there are two primary methods that we will 
discuss that are commonly applied within the TDDFT framework: real-time propagation and linear response approximation. Both methods are applied to our model systems to examine how these approximations and approximate functionals compare to exact solutions and their corresponding propagations.

\section{Real-time propagation}

Previously, we discussed how we assume our nuclei move on a slow enough time scale when compared to the electron that they can be approximated as being stationary. We called this an adiabatic approximation, due to the slowly varying nature of the nuclei allowing us to assume that we never leave an equilibrium state. In the same way, when we are time-evolving our electronic density, we can make a similar assumption that stabilizes our problem as we continue in time. Using the adiabatic approximation, we are able to make the statement that by assuming that the system is only slowly varying in time, our instantaneous potential reduces to the ground-state counterpart,

$$
v_{\mathrm{xc}}(\mathbf{r}, t) \rightarrow v_{\mathrm{xc}}[n(t)](\mathbf{r}, t)
$$

Assuming our Hamiltonian is not explicitly time-dependent, we can apply the time-evolution operator onto our KS ground state wavefunctions. Defining the timeevolution operator in the usual way,

$$
\hat{\mathcal{U}}(t)=e^{-i H t} \Longrightarrow\left|\varphi_{i}(t)\right\rangle=e^{-i H t}\left|\varphi_{i}(0)\right\rangle
$$

we have a prescription to observe how our density changes under a time-dependent external potential. In practice, appying the time-evolution operator as prescribed in 


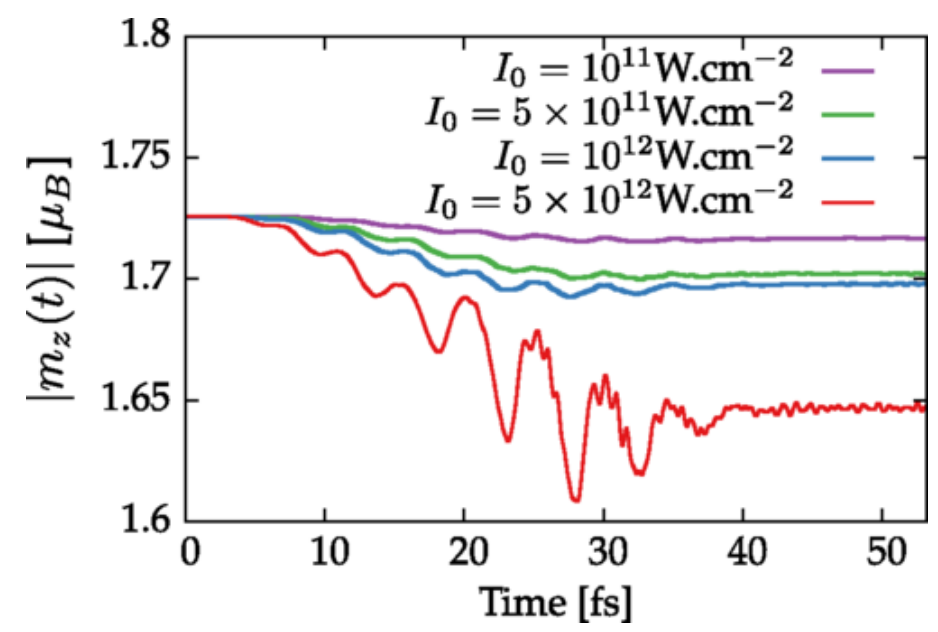

Figure 2.2: Time evolution of the magnetization integrated around $\mathrm{Ni}$ atoms for varying driving intesities in an $\mathrm{NiO}$ extended system. Adapted from [28]

Eq. (2.33) is extremely unstable. To fix this, we apply the Crank-Nicolson algorithm, defined as

$$
(1-i \mathbf{H} d t / 2) \varphi^{(k+1)}=(1-i \mathbf{H} d t / 2) \varphi^{(k)} .
$$

This can be interpreted as a "half-step" forward in time to average the difference between the previous half-step and the next time-evolved state, $\varphi^{(k+1)}$.

The model systems are stable under the use of Eq. (2.34), so we can stop there, however there are more sophisticated reformalized versions of the time-evolution operator that perform more efficiently in more complex systems [24-27]. That being said, real-time propagation is extensively used within the field of TDDFT with wideranging applications. Because we are directly recovering and monitoring the density, we can recover many observable quantities and directly monitor the density response to the perturbation. As we shall see in Chapters 4 and 5, by including spin dependence, one such quantity we can monitor is the magnetization of our system as it evolves in real time due to the perturbative laser field. 
Using these real-time methods gives then allows us to explore ultra-fast phenomena as they happen. For example, we can watch the real-time demagnetization and saturation of the magnetization of the $\mathrm{Ni}$ atoms in $\mathrm{NiO}$ shown in Figure 2.2. Although there are many uses of RT-TDDFT, real-time propagation in DFT is often used to calculate nonlinear excitation phenomena, such as high-harmonic generation [28-30]. Additionally, RT-TDDFT is commonly used to investigate stopping power and electron dynamics in solids and to explore electron dynamics in real-space grid modeling [31-33]. In this work, as mentioned in the introduction, we will focus on exploring the magnetization dynamics of our system using real-time propagation techniques to explore laser pulses hitting a triangular lattice system. A more thorough discussion of real-time applications and approaches can be found in Time-Dependent Density Functional Theory [34].

\section{Linear Response TDDFT}

Real-time propagation is undoubtedly useful and applicable to many systems, but physicists and chemists have a second method if they are seeking information about excited-state properties. In this way, a researcher doesn't have to worry about determining the correct time step that will be most computationally efficient, while not self-immolating in the process. Additionally, the electronic excitations are of particular interest in many molecular systems where calculating absorption spectra and the like are key to identifying the mechanisms of the reaction of the system to the external stimulus. The formulation comes from a transformation of variables from the time domain to the frequency domain. From this transformation, rather than directly monitoring the change in density through time, we immediately recover the 
excitation energies of our system and can recover the exact density difference between the states that are participating in a given excitation.

Suppose we have calculated our system's ground state, $n_{0}(\mathbf{r}) \Longrightarrow \varphi_{i}^{\mathrm{KS}}(\mathbf{r})$. We have eigenvalues for the energy levels for the occupied and unoccupied states. The key is to determine an equation or system of equations that can use those energy eigenvalues as input to allow us to calculate the orbital contributions to our excitations. To begin, we note we can invert our functionals such that:

$$
V_{\text {eff }}[n](\mathbf{r}, t) \rightarrow n\left[V_{\text {eff }}\right](\mathbf{r}, t)
$$

Expanding the density and truncating to first order, we get the linear density response:

$$
n_{1}(\mathbf{r}, t)=\int d t^{\prime} \int d^{3} r^{\prime} \chi_{s}\left(\mathbf{r}, t, \mathbf{r}^{\prime}, t^{\prime}\right) V_{\mathrm{eff}, 1}\left(\mathbf{r}^{\prime}, t^{\prime}\right)
$$

Where, $\chi_{s}$ is the density-density response function and $V_{\text {eff, } 1}$ is the linearized effective potential. Now, if we Fourier Transform our linear density response equation, we see that all terms become frequency dependent. Once the equation is transformed, we find that the density-density response function can be recast with frequency dependence below.

$$
\chi_{s}\left(\mathbf{r}, \mathbf{r}^{\prime}, \omega\right)=\sum_{j, k=1}^{\infty}\left(f_{k}-f_{j}\right) \frac{\varphi_{j}^{0}(\mathbf{r}) \varphi_{k}^{0 *}(\mathbf{r}) \varphi_{j}^{0 *}\left(\mathbf{r}^{\prime}\right) \varphi_{k}^{0}\left(\mathbf{r}^{\prime}\right)}{\omega-\omega_{j k}+i \eta}
$$

Here, $f_{j}$ and $f_{k}$ denote KS ground-state orbitals (with 0 and 1 corresponding to unoccupied and occupied states, respectively) and $\omega_{j k}$ is the difference between KS energy eigenvalues. Clearly, this tells us that the excitation energies correspond to the poles of Eq. (2.37). 
Now that we know what our density-density response function looks like, we can take a step back into our linear response equation. After transforming into the frequency domain, we are left with the equation below, where $\Omega$ relates to the exact energy eigenmode of the system.

$$
n_{1}(\mathbf{r}, \Omega)=\int d^{3} r^{\prime} \chi_{s}\left(\mathbf{r}, \mathbf{r}^{\prime}, \Omega\right) \int d^{3} r^{\prime \prime} f_{\mathrm{Hxc}}\left(\mathbf{r}^{\prime}, \mathbf{r}^{\prime \prime}, \Omega\right) n_{1}\left(\mathbf{r}^{\prime \prime}, \Omega\right)
$$

Above, $f_{H x c}$ is Hartree exchange-correlation kernel. Here, the Hartree term is just added to $f_{x c}$ as seen below.

$$
f_{\mathrm{Hxc}}\left(\mathbf{r}, \mathbf{r}^{\prime}, \omega\right)=\frac{1}{\left|\mathbf{r}-\mathbf{r}^{\prime}\right|}+f_{\mathrm{xc}}\left(\mathbf{r}, \mathbf{r}^{\prime}, \omega\right)
$$

Additionally, we can write the linear response in matrix form. Now, making a few clever substitutions and doing some algebra, we can rewrite our linear density response in the form of an eigenvalue problem.

$$
\begin{gathered}
\sum_{i^{\prime} a^{\prime}}\left(\left[\delta_{i i^{\prime}} \delta_{a a^{\prime}} \omega_{a^{\prime} i^{\prime}}-K_{i a, i^{\prime} a^{\prime}}\right] X_{i^{\prime} a^{\prime}}+K_{i a, a^{\prime} i^{\prime}} Y_{i^{\prime} a^{\prime}}\right)=-\Omega X_{i a} \\
\sum_{i^{\prime} a^{\prime}}\left(K_{a i, i^{\prime} a^{\prime}} X_{i^{\prime} a^{\prime}}+\left[\delta_{a a^{\prime}} \delta_{i i^{\prime}} \omega_{a^{\prime} i^{\prime}}+K_{a i, a^{\prime} i^{\prime}}\right] Y_{i^{\prime} a^{\prime}}\right)=\Omega Y_{i a}
\end{gathered}
$$

Importantly, if our KS orbitals are real, we can recast these eigenvalue equations into a compact matrix formalism, also known as the Casida Equation.

$$
\left(\begin{array}{ll}
\mathbb{A} & \mathbb{B} \\
\mathbb{B} & \mathbb{A}
\end{array}\right)\left(\begin{array}{l}
\mathbf{X} \\
\mathbf{Y}
\end{array}\right)=\Omega\left(\begin{array}{cc}
-\mathbb{I} & 0 \\
0 & \mathbb{I}
\end{array}\right)\left(\begin{array}{l}
\mathbf{X} \\
\mathbf{Y}
\end{array}\right)
$$


Here, matrices $\mathbb{A}$ and $\mathbb{B}$ are given by the following equations, which come from simplifying Eq. (2.36).

$$
\begin{gathered}
A_{i a, i^{\prime} a^{\prime}}(\Omega)=\delta_{i i^{\prime}} \delta_{a a^{\prime}} \omega_{a i^{\prime}}+K_{i a, i^{\prime} a^{\prime}}(\Omega) \\
B_{i a, i^{\prime} a^{\prime}}(\Omega)=K_{i a, i^{\prime} a^{\prime}}(\Omega)
\end{gathered}
$$

Here, our $K_{i a, i^{\prime} a^{\prime}}$ matrix is equivalent to the following integration, where $f_{\mathrm{Hxc}}$ is assumed to be frequency independent,

$$
K_{i a, i^{\prime} a^{\prime}}=2 \int d^{3} r \int d^{3} r^{\prime} \Phi_{i a}^{*}\left(\mathbf{r}^{\prime}\right) f_{\mathrm{Hxc}}\left(\mathbf{r}, \mathbf{r}^{\prime}\right) \Phi_{i^{\prime} a^{\prime}}(\mathbf{r})
$$

where $\Phi_{i a}(\mathbf{r})=\varphi_{i}^{*}(\mathbf{r}) \varphi_{a}(\mathbf{r})$ where $i$ and $a$ run over the occupied and unoccupied orbitals, respectively. Solving the system of Eqs. (2.40) and (2.41) or Eq. (2.42), we solve for our eigenmodes, associated with a given frequency $\Omega$.

$$
n_{1}(\mathbf{r}, \Omega)=\sum_{i a}\left[\Phi_{i a}^{*}(\mathbf{r}) X_{i a}(\Omega)+\Phi_{i a}(\mathbf{r}) Y_{i a}(\Omega)\right]
$$

Importantly, the density response is also known as the transition density associated with the frequency $\Omega$. In TDDFT the Kohn-Sham TDM is obtained as a superposition of TDMs associated with single particle excitations. Therefore, we can rewrite our transition density equation as a matrix for each of these transition states.

$$
\Gamma_{n}\left(\mathbf{r}, \mathbf{r}^{\prime}\right)=\sum_{i a}\left[\varphi_{i}(\mathbf{r}) \varphi_{a}^{*}\left(\mathbf{r}^{\prime}\right) X_{i a}^{(n)}+\varphi_{i}^{*}\left(\mathbf{r}^{\prime}\right) \varphi_{a}(\mathbf{r}) Y_{i a}^{(n)}\right]
$$

Now, we see that $\Gamma_{n}$ is the Kohn-Sham transition density matrix associated with the $n^{\text {th }}$ excitation of the system. Again, $\mathbf{X}$ and $\mathbf{Y}$ are the eigenvectors of the $n$-th 
excitation that are found from solving for the frequency eigenmodes, $\Omega$, of the system. Importantly, when $\mathbf{r}=\mathbf{r}^{\prime}$ we recover the density response of the transition state, Eq. (2.46). As we will see, using this Casida formalism will be advantageous to quickly

calculate excitation energies for our model system. Additionally, we can manipulate and transform the transition density, $n_{1}(\mathbf{r}, \Omega)$, through unitary transformations on the underlying Kohn-Sham basis.

\subsection{Bringing it all together: model systems}

As can be seen from the previous sections, the metaphorical house we are building has been framed, the foundation has been poured, and the roof tarred and thatched. With this sturdy structure created, we can now begin to paint the interior and decorate our rooms to upgrade a simple habitat into a home. To that end, we must connect where we've been to where we will go.

Physicists are reviled and praised for their ability to shape a problem: taking a complex and almost untenable task and simplifying it to the point that mathematicians would blush and scream concomittantly. Here, we will show that this is not an altogether despicable thing. Rather, we aim to show that simple model systems, where complexity has been reduced through careful parameter selection or physical assumptions, can in fact elucidate and illuminate the path towards understanding our physical world. 


\subsubsection{Returning to the quantum world}

Here, we begin by going back the building blocks of every physicists journey into the subject matter: introductory quantum mechanics. What we will now venture into is both the first lesson most students experience in those quantum classes and one of the most simplistic models possible. Because of its simplicity and approachability, the quantum well and its extensions become an ideal starting place to understand the impact model systems can have on our study of physics.

\section{The quantum well}

Surprisingly, the humble quantum well that each physicist learns is one area of research that has become nearly ubiquitous in modern life, and few people other than physicists realize it. In America, at least during times that are removed from the events of 2020 (see: Coronavirus), enter into the average citizen's home, and one would likely find some sort of television in the house. Perhaps even more likely, if their phone number were requested, they would pull out a cell phone, perhaps leveraging the technology that takes advantage of the physics that every current graduate student learned prior to matriculating into their program.

What the two technologies above share are screens, and the ability to emit light from their components. In modern day society, one area of physics that is popular and constantly present are the use of quantum dots, especially in the aforementioned screens for many electronic devices. While that is the most readily visible application of quantum dots, Figure 2.3 shows that there are many avenues in which quantum dots have been applied or their use is currently being investigated. The reason for their popularity is their relative cost and tunability, but perhaps surprisingly, the physics 


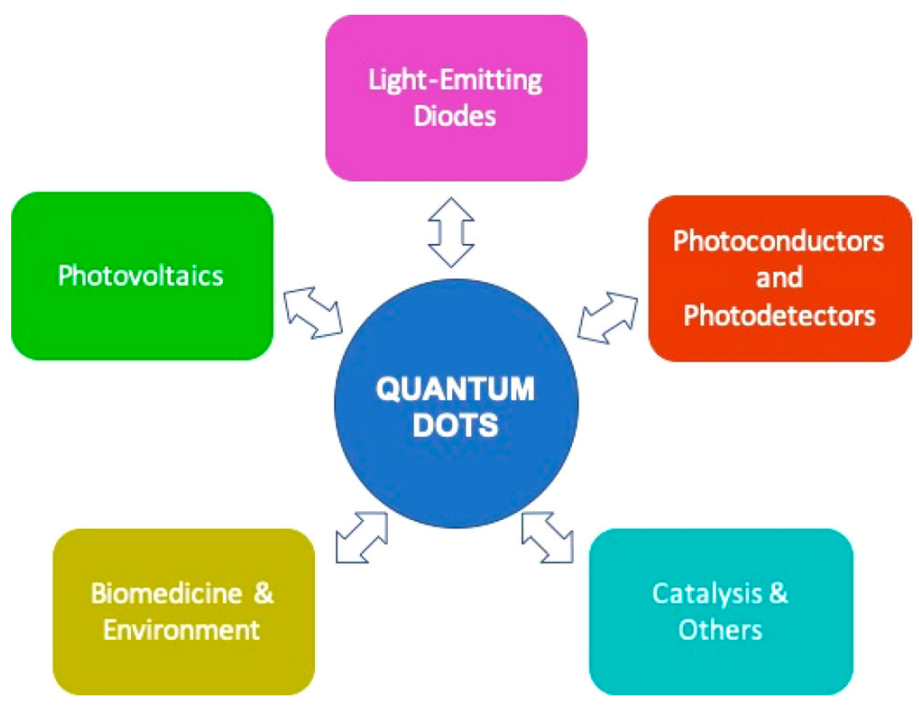

Figure 2.3: Current areas of research where quantum dots are being applied or actively explored. Adapted from [35]

taking place in quantum dots is studied by every physics major across the world in the form of the particle-in-a-box problem. While the particle-in-a-box model could never fully capture all of the intricacies of material selection or device efficiency that are crucial to create a product that sells well to the masses, it admirably and easily explains the physics of the system in a way that is easily generalized and understood.

To begin, we start with the stereotypical particle-in-a-box, where the well is defined with infinite potential walls and is of length $L$. By defining our potential in this way, we are left with a Hamiltonian that looks like the following,

$$
\hat{H}=-\frac{1}{2} \frac{\partial^{2}}{\partial x^{2}}+V(x)
$$

Traditionally, $V(x)$ is set to 0 for $0<x<L$, else $V(x)=\infty$. The Schrödinger equation that we get from Eq. (2.48) is straightforward and when we solve for the 


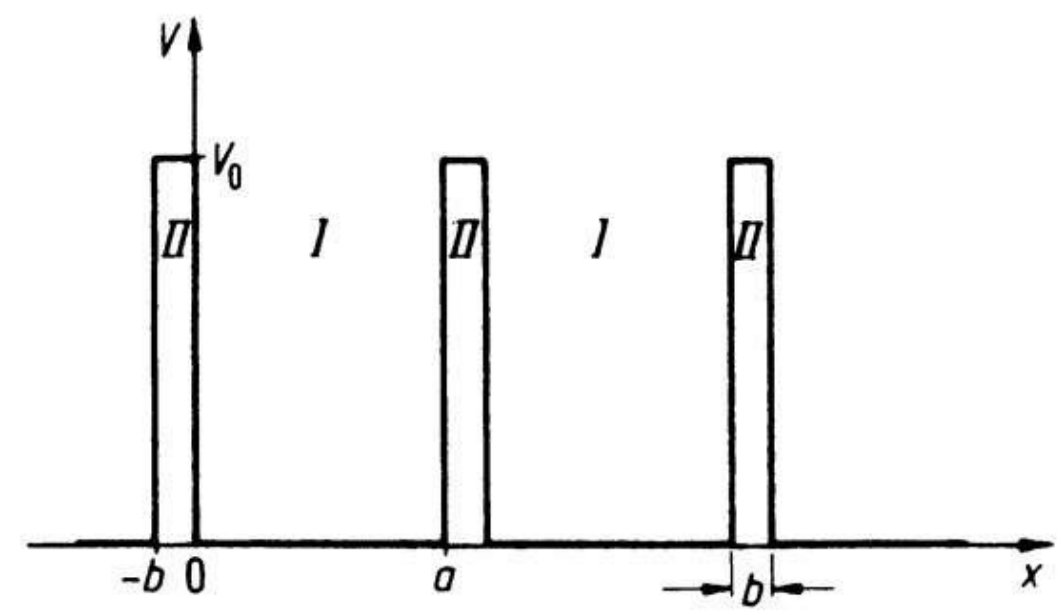

Figure 2.4: The Kronig-Penney potential. A repeating set of finite potential wells with a potential of $V_{0}$ of width $a$ and barrier widths of $b$ for each well. Note that the wells extend on into infinity in $x$.

boundary conditions and the energy, we get

$$
E_{n}=\frac{(n \pi)^{2}}{2 L^{2}} .
$$

So, we have arrived at the solution that every physicist knows and loves, but how does this get us anything as physically rich as a quantum dot?

To do that we must apply more realistic boundary conditions to our system by using finite potentials. Again, we have a box of identical shape to the particle-in-a-box example, but replace our infinite boundaries with finite counterparts. The solution is left as an exercise for the reader, but what is important is by introducing finite potentials, our electron now has some nonzero probability density outside of our box. This means our particle can now "tunnel" through the barrier and escape our region of confinement. Put another way, our electronic wavefunction now has some overlap with the barrier. 
If we expand this idea further and create periodic wells that are regularly distributed along our axis, we can see that we've begun to create something similar to a periodic solid. Solving this repeating system of wells, which is known colloquially as the Kronig-Penney model and is visualized in Figure 2.4, we can generalize a simple particle-in-a-box up to a model of a one-dimensional solid. Rather than a wavefunction where our distribution is only within the well or exponentially decaying through across our barrier, we now have a function that will repeat with the periodicity of our wells. This solution is known as a Bloch wavefunction and can be mathematically written as

$$
\psi(x)=e^{i k \cdot x} u(x)
$$

After inserting the Bloch function into our Schrödinger equation, we can solve our differential equation and match up our functions at the boundaries as in the finite well problem. Once that is done, we are left with the following equation,

$$
V_{0} a b \frac{\sin (\alpha a)}{\alpha a}+\cos (\alpha a)=\cos (k a)
$$

where $a$ is the width of the well and $b$ is the separation between two wells. Also, $\alpha$ is defined to be $\sqrt{2 E}$.

Immediately, we see that there are quantizations of the values which $\alpha a$ is allowed to take because of the $\cos (k a)$ term on the right hand side of Eq. (2.51). Rather than discrete values, as we saw was the case for the particle-in-a-box example, we instead of have quantized areas (bands), where we have allowed energetic solutions. If we imagine increasing the barrier height, $V_{0}$, we can see that we would get sharper (smaller) bands of allowed values and the counterpositive also holds. As $V_{0}$ approaches 


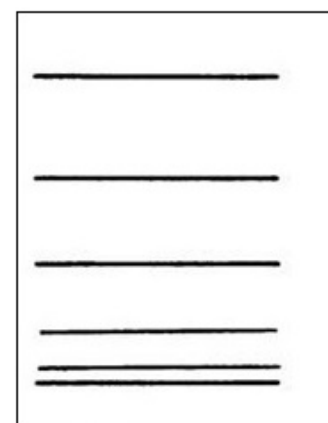

(a)

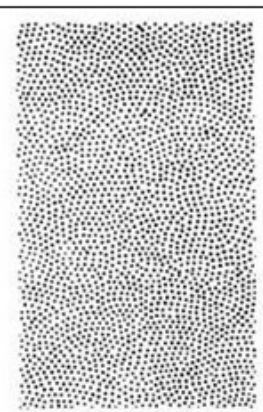

(b)

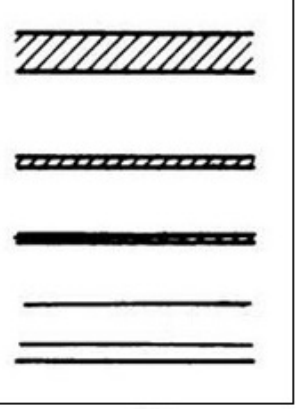

(c)

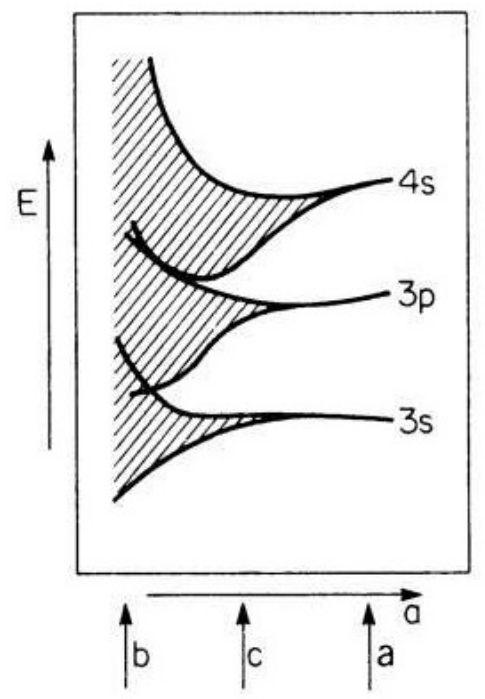

Figure 2.5: Schematic plots showing (left) allowed energy levels for (a) single, infinite wells, (b) free particles, and (c) periodic finite potential wells (the Kronig-Penney potential). (Right) how energy dispersion changes with increasing interatomic distance. We go from the (a) continuous, free particle region to (b) discrete bands, and finally to (c) discrete, quantized energy levels [36]. 
infinity, we also approach the quantized results that we saw in our particle-in-a-box example. Thus, our extended system leaves its energy band configuration and appears to approach the behavior of a finite, confined particle behavior. Schematically, we can draw this like Figure 2.5, where our band dispersions diminish towards quantized energy levels with increasing interatomic distance.

Now that we've laid the table with the basics, we can see that if we can effectively confine the electrons in space, by either increasing the potential barrier or by increasing the interatomic distance, we approach what looks like discretized atomic energy levels. So, as we quantum mechanically confine the electron to a small space, we should expect to get behavior that is similar to what we see in the well-defined energy levels seen in molecules and atoms. Indeed, this is why quantum dots are often referred to as artificial atoms: we are confining electrons spatially such that they behave in the same way energetically that they would in an atom. An example of the discretized energy states can be seen for a GaAs quantum dot in Fig. 2.6, and it can be seen that adding a magnetic field, the energy needed to introduce a new electron increases and generally overlaps with the energy needed to add an additional electron with no magnetic field. Theoretically and experimentally, this behavior begins to display whenever the size of the system being explored is on the same order as the exciton Bohr radius,

$$
r_{n}=\left(\frac{m_{0} \varepsilon_{r} a_{\mathrm{H}}}{\mu}\right) n^{2},
$$

where $m_{0}$ is the electron mass, $\varepsilon_{r}$ is the relative permittivity of the material, and $\mu$ is the reduced mass of the electron-hole pair $-m_{e}^{*} m_{h}^{*} /\left(m_{e}^{*}+m_{h}^{*}\right)$.

In practice, this means that if a researcher is able to confine an electron in some way, we can actually fairly accurately predict what the electron dynamics will be. 


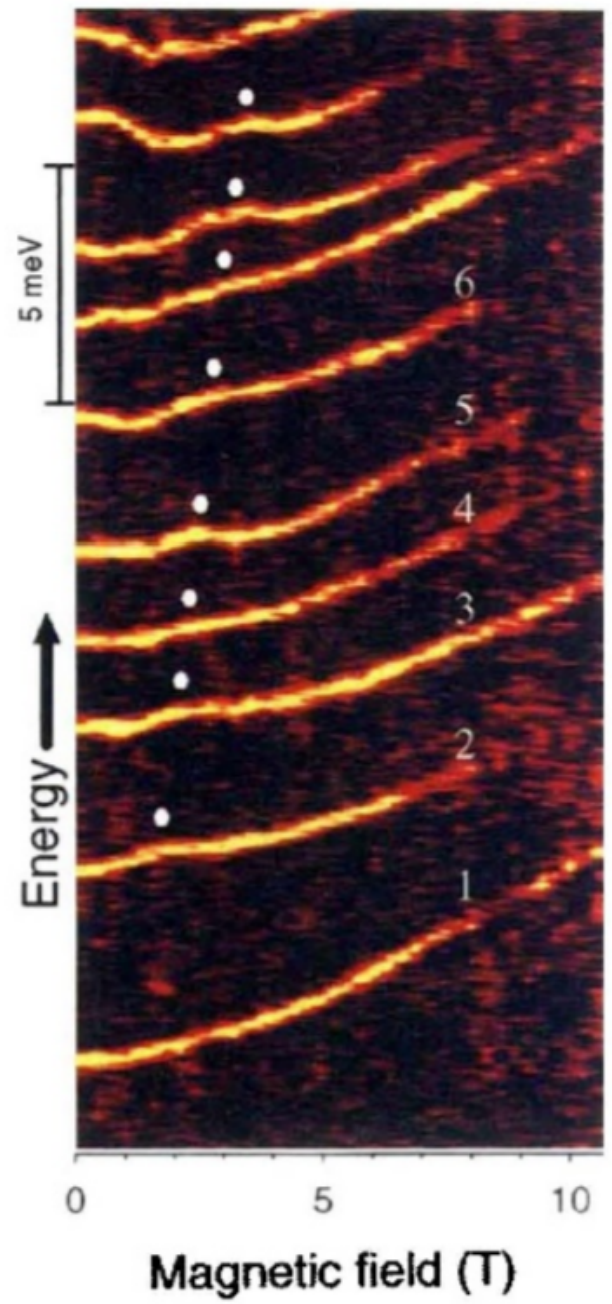

Figure 2.6: Single-electron capacitance spectroscopy experiment measuring the energy to add an electron to an artificial atom made of GaAs. The yellow lines correspond to the energy needed to add an electron into the quantum dot. The first six energy levels are numbered and the white dots correspond to spontaneous spin alignment of the electrons in the quantum dot. Adapted from [37]. 

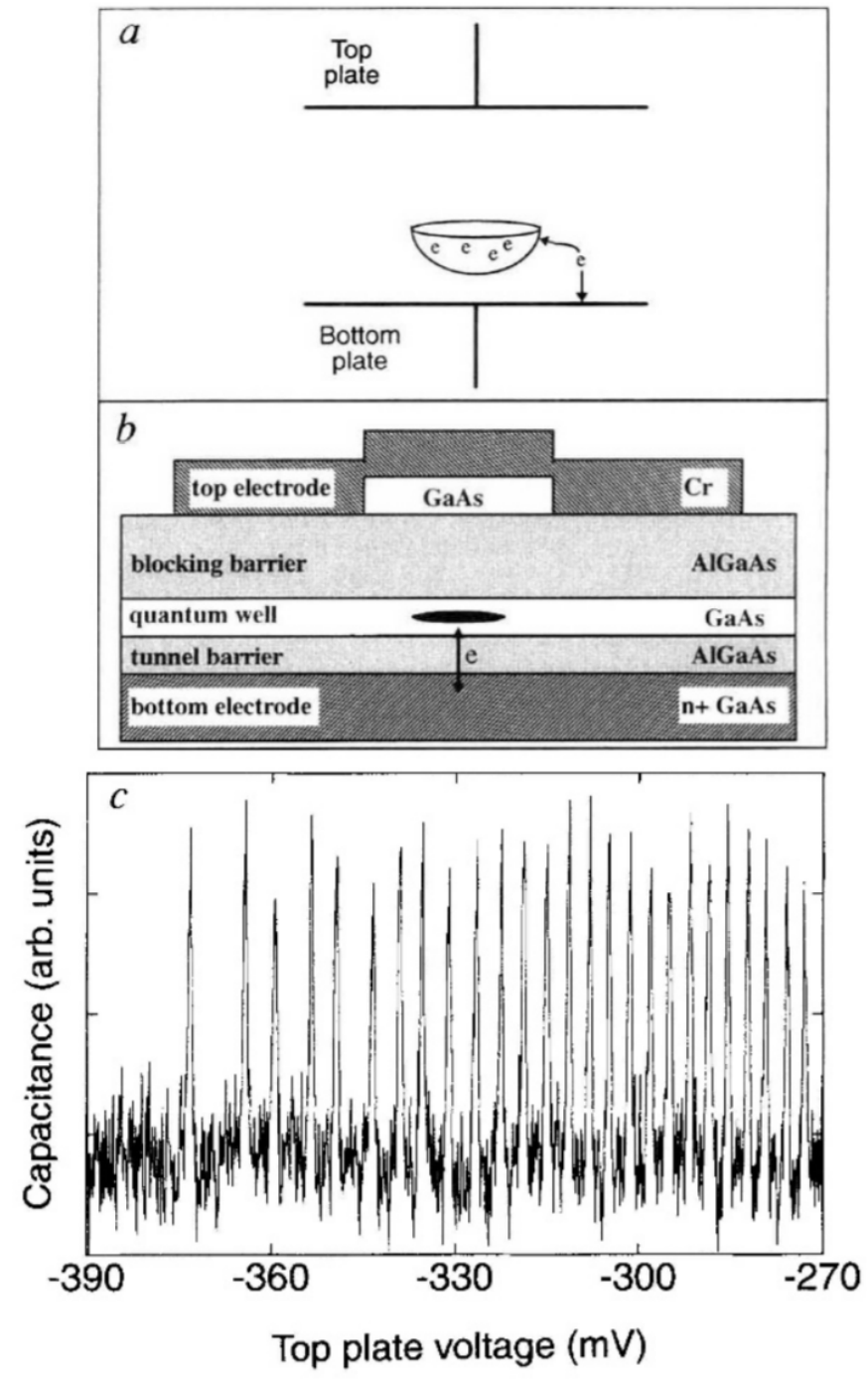

Figure 2.7: Schematic depiction (a) of the paraboloid potential of a quantum dot. An experimental sample quantum dot (b) created by sandwiching GaAs between two AlGaAs layers with an artificial atom represented by the black disk. Finally, the measured capacitance (c) of the experimental GaAs sample measured as a function of the plate voltage. The peaks in the capacitance correspond to electrons entering the atom. Adapted from [37] 
Obviously, real life corrections must be made and there has been extensive study on the shape of confinement and magnetic field effects on energy levels and electronic behavior, but on a basic level quantum dots can be approached as an application of a particle-in-a-box [38]. Still, quantum dots are extremely popular areas of research because of their broad applicability and relative ease with which they can be fabricated [35]. For example, we can see a GaAs quantum well created by sandwiching the GaAs between AlGaAs in Figure 2.7. Single electrons can be added to the dot by adjusting the gate voltage and the electron is confined in this system to the GaAs layer, which is subject to a two dimensional paraboloid potential. While simplistic in nature, and only a small step removed from the one-dimensional particle-in-a-box example we solved previously, the potential compares favorably to experiments [37,39-41].

\section{The tight-binding model}

Taking a step beyond the applications of a quantum well, we can move to a lattice system. Underlying the study of all of condensed matter, as we made clear in Section 2.1.1, is the idea that nuclei are close to stationary when compared to the movement of electrons, especially within a solid. It was fairly easy to reconcile the idea of clamping the nuclei in place because of their relative mass compared to electrons, and thus the time scales of their movements are long in the reference frame of the electron. But, could we simplify and restrict the motion of the electrons as well? After all, we know the potential fields exerted on our small, negatively charged friends are what define their motion.

We might assume then, that with the relatively vast difference in size between electrons and nuclei that the electrons are more strongly influenced by the presence and 
placement of the nuclei when compared to their fellow electrons. To mathematically implement this idea, we assert that electrons prefer to stay isolated and in close proximity to their atomic centers, occasionally jumping to another atom, while neglecting the electron-electron interactions. Assuming we have a crystal/lattice structure, we can formulate our Hamiltonian as

$$
\hat{H}=\hat{H}_{\text {atom }}+\Delta U,
$$

where $\hat{H}_{\text {atom }}$ is defined as a Hamiltonian describing an independent atom and $\Delta U$ is the potential difference due to the crystal/lattice structure. As we approach an atomic center, we expect that the potential from that local atom will dominate the potential coming from other atomic sites, which implies $\Delta U \rightarrow 0$ as the electronic position $r$ approaches $R_{j}$ (the location of our $j$-th nuclei).

Having defined our global Hamiltonian, we should investigate how we define our atomic Hamiltonian. If we assume that our lattice sites can be separated far enough in space, that is that the potential wells between lattice/atomic sites do not overlap, we can define our atomic Hamiltonian such that the eigenstates of each atomic site are the atomic eigenstates/wavefunctions. Mathematically, we are left with the Schrödinger equation that describes are atomic states,

$$
\hat{H}_{\text {atom }} \phi_{i}(\mathbf{r})=\varepsilon_{i} \phi_{i}(\mathbf{r}) .
$$

By defining our wavefunctions as atomic orbitals, we can describe our system with wavefunctions that are separated in space, meaning the overlap of wavefunctions 


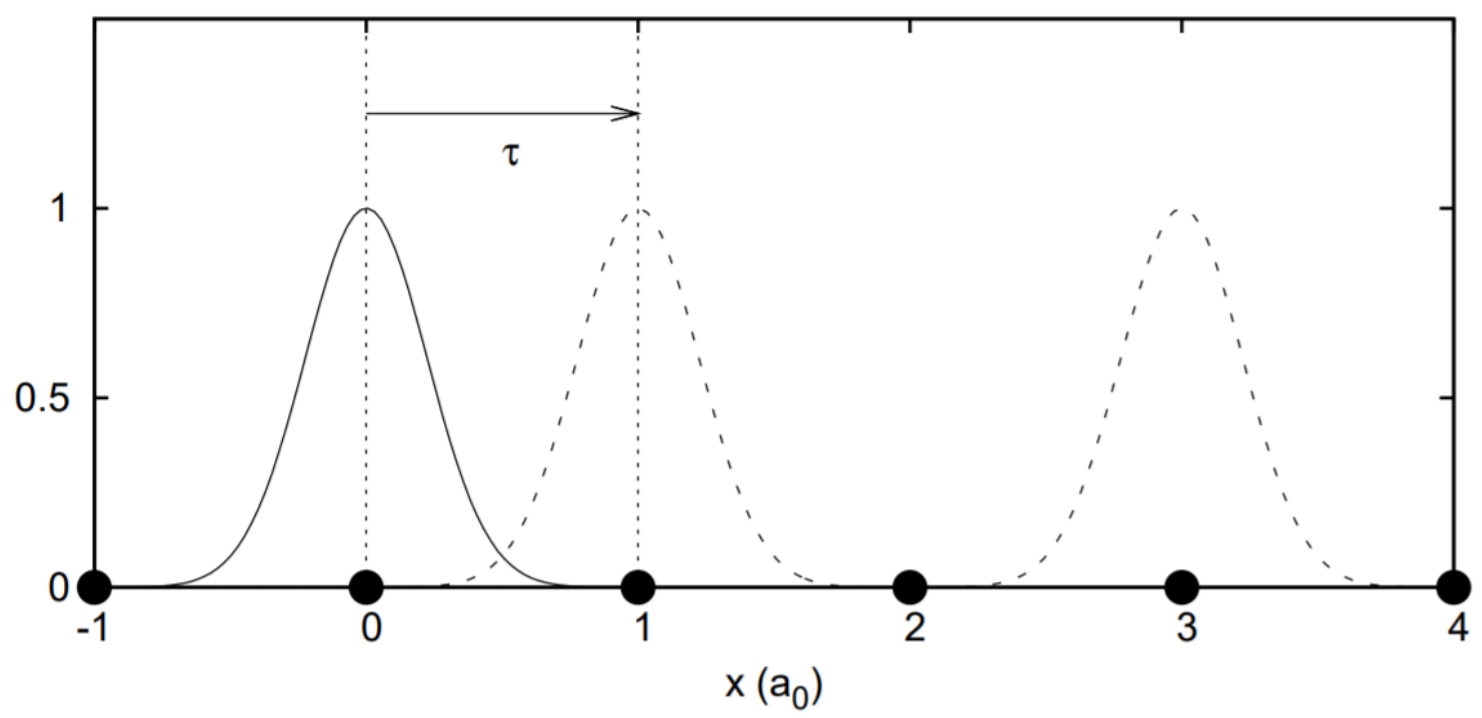

Figure 2.8: Diagram of the atomic orbitals in a one-dimensional crystal separated by units of $a_{0}$. The atomic orbitals are described by the solid and dashed lines. The vertical dashed line demarcate the edges of the unit cell of our one-dimensional lattice and $\tau$ is the vector denoting the nearest neighbor to site 0. Equation (2.55) denotes the area contained beneath the solid and dashed wavefunctions in the region between the 0-th and 1-st lattice sites. Schematic reproduced from [42]. 
between neighboring lattice sites/atoms are small. We define this overlap integral as

$$
\int \phi_{i}^{*}(\mathbf{r}) \hat{H} \phi_{i}(\mathbf{r}+\mathbf{R}) d \mathbf{r}
$$

where $\phi_{i}(\mathbf{r}+\mathbf{R})$ is the $i$-th wavefunction of the atom located a distance $\mathbf{R}$ from our origin atom. In other words, we assume that $\mathbf{R} \neq 0$ in this formulation. Schematically, we can visualize this equation as Figure 2.8, where we have wavefuctions emanating radially from the atomic centers and overlapping slightly with neighboring atomic wavefunctions. By using these local atomic orbitals as our basis, we can still satisfy Eq. (2.50), retrieving wavefunctions that can describe the periodic nature of a solid and capture band calculations.

Indeed, even a simplistic model such as the tight-binding model provides the theoretical framework of interesting phenomena currently being discussed in physics. Perhaps the best known example of this would be the material graphene. We begin where we left off above, we have Bloch wavefunctions describing our tight-binding system, which are explicitly written as

$$
\Phi_{j}(\mathbf{k}, \mathbf{r})=\frac{1}{\sqrt{(} N)} \sum_{i=1}^{N} e^{i \mathbf{k} \cdot \mathbf{R}_{j, i}} \phi_{j}\left(\mathbf{r}-\mathbf{R}_{j, i}\right)
$$

where our $\phi_{j}$ are the atomic wavefunctions for each site located in our unit cell. Redefining our basis, we can get the electronic wavefunction as a linear combination of our Bloch functions,

$$
\Psi_{j}(\mathbf{k}, \mathbf{r})=\sum_{l=1}^{n} c_{j, l}(\mathbf{k}) \Phi_{l}(\mathbf{k}, \mathbf{r}) .
$$

That leaves us with our Schrödinger equation from which we can calculate the energy of each electron band. Solving our energy, and substituting the wavefunctions from 
our new Bloch wave basis, we recover

$$
E_{j}(\mathbf{k})=\frac{\sum_{i, l}^{n} H_{i l} c_{j i}^{*} c_{j l}}{\sum_{i, l}^{n} S_{i l} c_{j i}^{*} c_{j l}},
$$

where $H_{i l}=\left\langle\Phi_{i}|\hat{H}| \Phi_{l}\right\rangle$ and $S_{i l}=\left\langle\Phi_{i} \mid \Phi_{l}\right\rangle$ is the overlap integral. For graphene, specifically, we have two orbitals per unit cell, which means we are left with the following equation

$$
H \psi_{j}=E_{j} S \psi_{j}
$$

which we can diagonalize to solve for the wavefunctions of our system.

It is important to note that $H$ and $S$ are still matrices in this case, each with 4 quantities, which can be calculated by plugging Eq. (2.57) into the definition of our matrices from Eq. (2.58). By assuming a tight-binding framework, we can assume that our wavefunctions only overlap slightly with the nearest neighbor orbitals. That means, there are no contributions in our overlap integrals from any wavefunctions other than the 3 sites that surround each sublattice point in the customary A-B sublattice definition of graphene visualized in Figure 2.9. Calculating the energies from these equations is mainly an exercise in geometry and once calculated, we recover our band equation equations for graphene,

$$
E_{ \pm}=\frac{\varepsilon_{2 p} \pm \gamma_{0}|f(\mathbf{k})|}{1 \mp s_{0}|f(\mathbf{k})|}
$$

where $\varepsilon_{2 p}=\left\langle\phi_{j}|\hat{H}| \phi_{j}\right\rangle, \gamma_{0}$ are the AB overlap integrals, and $f(\mathbf{k})=e^{i k_{y} a / \sqrt{(3)}}+$ $2 e^{-i k_{y} a / 2 \sqrt{(3)}} \cos \left(k_{x} a / 2\right)$.

So, we can determine the band structure of graphene in the tight-binding frame- 


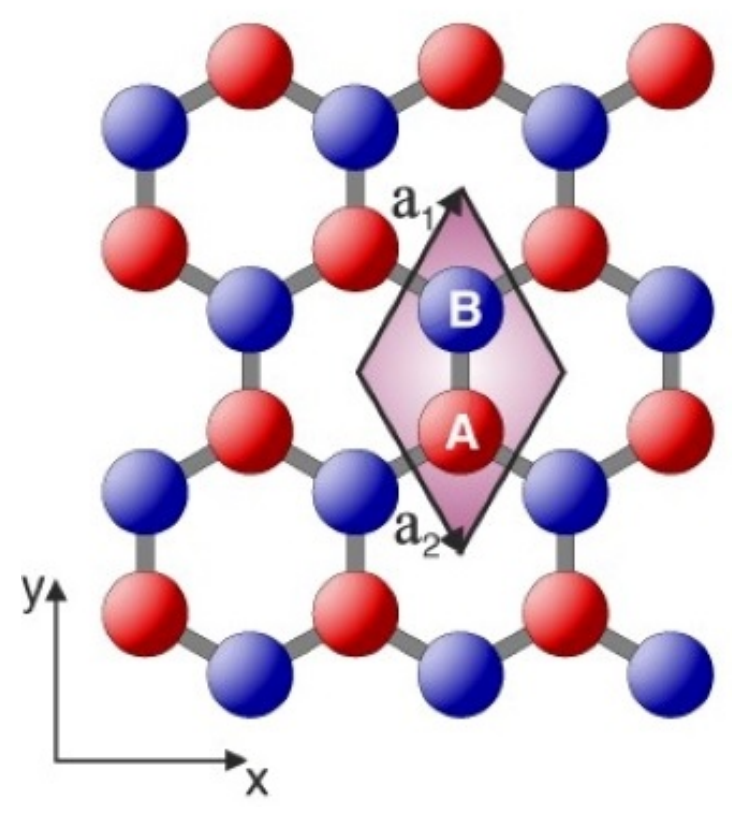

Figure 2.9: Schematic of a graphene lattice with A-B sublattice labeling and corresponding colors, red and blue, respectively. The unit primatives are labeled $a_{1}$ and $a_{2}$ with the unit cell shown as the shaded region. Adapted from [43] 


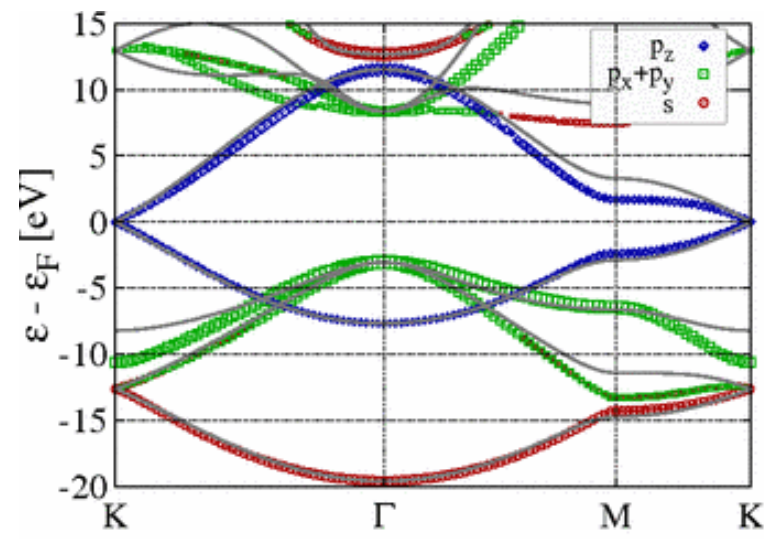

Figure 2.10: Band structure calculations of graphene with spin-orbit coupling for an atomic orbital basis for tight-binding calculations (gray lines) and with densityfunctional theory (colored markers) using a linearized augmented plane-wave basis. The DFT calculations were done with Wien2k using the PBE functional. The bands are colored according to their atomic orbital and the Dirac cones can be seen at the $K$ points on the edges of the plot. Adapted from [44].

work, but how does that compare to experimental results? In Figure 2.11, the experimental results confirm the Dirac cones that are predicted by tight-binding model. Likewise, Figure 2.10 shows that the band structure calculated with more complex DFT methods using including spin-orbit coupling compare favorably to tight-binding model results (with spin-orbit coupling included).

We have shown that the tight-binding model has good predictive power and is in good agreement with both calculation and experiment, as we displayed in Figures 2.11 and 2.10, but it is also an important foundation for other theories. One of those theories and models forms the basis and foundation upon which this thesis is built. Recall that in Eq. (2.55), we wrote the overlap integral in the single, atomic orbital basis. Beginning from this localized basis, we can then reorganize Eq. (2.56) in terms of creation and annihilation operators in the second quantization formalism, leaving 
us with

$$
c_{\mathbf{k} \sigma}=\frac{1}{\sqrt{(N)}} \sum_{i} e^{i \mathbf{k} \cdot \mathbf{R}_{i}} c_{i \sigma}, c_{\mathbf{k} \sigma}^{\dagger}=\frac{1}{\sqrt{(N)}} \sum_{i} e^{-i \mathbf{k} \cdot \mathbf{R}_{i}} c_{i \sigma}^{\dagger}
$$

Here, the creation operator $c_{i \sigma}^{\dagger}$ creates an electron in the corresponding $\phi\left(\mathbf{r}-\mathbf{R}_{i}\right)$ orbital with spin $\sigma$, while $c_{i \sigma}$ annihilates the electron from the same $i$-th orbital with spin $\sigma$. By reformulating our equations in terms of our second quantization operators and including the Coulombic repuslion term between electrons, we are able to recover (with some hidden algebra) the Hamiltonian in terms of our operators as

$$
H=\sum_{i, j} \sum \sigma\left(t_{i j} c_{i \sigma}^{\dagger} c_{j \sigma}+\text { h.c. }\right)+\sum_{i, \sigma} U c_{i \sigma}^{\dagger} c_{i \sigma} c_{i-\sigma}^{\dagger} c_{i-\sigma}+\sum_{i} \sum_{\sigma \sigma \prime} v_{i \sigma \sigma}^{\mathrm{ext}} c_{i \sigma}^{\dagger} c_{i \sigma \prime}
$$

Recall that previously, we defined the overlap integral in Eq. (2.55) and showed that in the highly localized limit, this quantity is small when we get far away from the atomic centers. If we examine that equation, we can see that when we switch to the second quantization formalism, that overlap integral is precisely equal to our $t_{i j}$ term. So, $t_{i j}$ behaves as our overlap integral between atomic sites, serving as a hopping term that a defines the likelihood that a particle will (or can) jump from site $i \rightarrow j$. That means, going from the extended system formalism, we can actually describe a finite system, where the lattice sites are locally defined quantities (like atomic orbitals or Wannier functions). The Hubbard Hamiltonian also ignores all electron-electron interactions (as the energies of those actions are small) except for the Coulombic repulsion term between electrons at the same lattice sites. This term is described by the $U$ term in Eq. (2.62) and is the quintessential approximation that makes the Hubbard model.

This profound idea means that we now have a simple model system that can be 

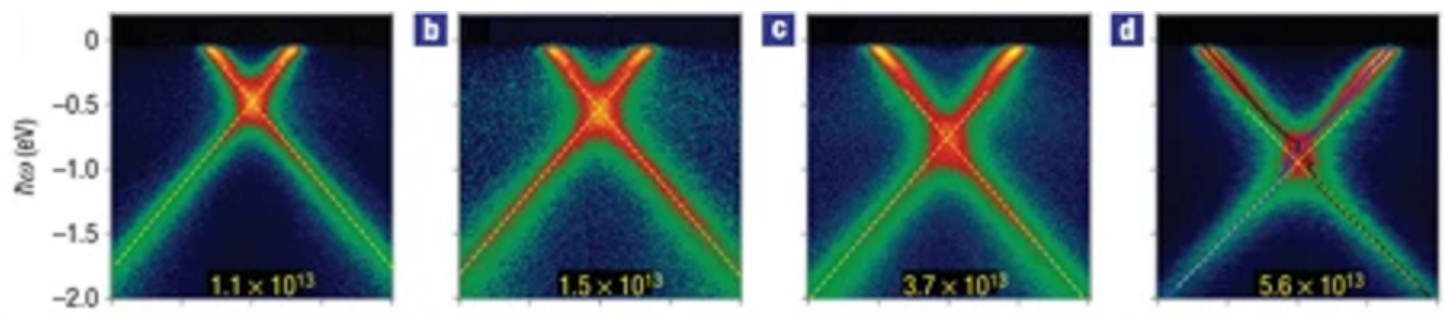

Figure 2.11: ARPES measurement of energy bands in graphene with increasing doping of Potassium along the $K$ point and parallel to the $\Gamma M$ direction. The electron density per $\mathrm{cm}^{2}$ is given on the bottom of each doping level. Clearly visible is the Dirac point where the conduction and valence bands meet for single layer graphene near the Fermi level. Adapted from [45].

viewed as discrete lattice sites with entirely localized quantities where the electronic density only sits on the defined sites, with no spatial distribution outside of those discrete sites. Interestingly, this definition came from an extended state formalism where the discrete sites can also be interpreted as band interactions within an extended system and the Hubbard model can be interpreted as systems where only the band near the Fermi energy participates in the electronic dynamics of the system. In practice the Hubbard model has been used to model Mott insulators and high-temperature superconductors [46]. And as the impetus for what a model system can and should be, I will leave the importance of the Hubbard model to the world of physics left said by the editors of Nature Physics [46]:

Although the Hubbard model secured its place in (condensed-matter) physics textbooks many decades ago, it is very likely that it will continue to play an important role in fundamental research as well. In particular, the continuing experimental progress in artificial lattices of cold atoms and superconductivity, where the Hubbard model and its modifications play a prominent role, should be a stimulus for further explorations. 
Simply put, the Hubbard still enjoys status as an influential model being studied and exposing interesting physical phenomena even today. The proceeding chapters will build upon the Hubbard formalism, adding interesting physical quantities such as the next-nearest neighbor interaction terms and spin-orbit coupling, but the model remains the simple and beautiful system that has spawned research and illuminated physical intuition. To finish with one final quote from Nature Physics again [46]:

Ever since its inception, the model has spawned new lines of research in theoretical physics; the development of dynamical mean-field theory is a noteworthy example. Although the model quickly became a firm favourite of theorists, it twice experienced a sudden rise in popularity due to breakthroughs in experimental physics ... Part of the legacy of Hubbard's model is that it launched the field of strongly correlated systems — it is undoubtedly the archetypal model of many-body physics.

And with that, we build upon the models that have illuminated the field of condensed matter physics and look to add a few more lumens that will make the world around us just a little more clear. 


\section{Chapter 3}

\section{The Localized Particle-hole Map}

As a disclaimer, much of this chapter is taken verbatim from the work of E. A. Pluhar and C. A. Ullrich, EPJB 91, 137 (2018) [47].

\subsection{Introduction}

Recent years have witnessed a proliferation of highly efficient organic semiconducting materials for use in photovoltaics and other areas of optoelectronics. Importantly, these devices rely on charge-transfer characteristics that are either inherent to the material or are emergent from the combination of two materials [48]. While many of the organic materials that display these characteristics have been known for years, timedependent density-functional theory (TDDFT) $[22,34,49,50]$ has now been established as the standard theoretical tool to analyze and predict their optical properties.

Since many of the organic materials of interest are quite complex, the question of visualizing the electron dynamics within these system remains a challenge. To this 
end, many tools to visualize charge dynamics have been developed in the literature, such as the time-dependent electron localization function [51] or the transition density matrix (TDM) [52-55]. The TDM is an approach based on the reduced one-body density matrix [56-59]; it can be used to isolate each transition state and generate a matrix representation that describes the density dynamics of the system in real space. Because of the nonlocal variables introduced in calculating the TDM, exciton coherence and delocalization lengths can be extracted from it [60]. Additionally, the TDM is the basis used to construct natural transition orbitals [61].

Previously, the particle-hole map (PHM) was introduced as a tool that elucidates even more electron dynamic information from a system, especially when used in conjunction with the TDM [62-65]. The PHM's key feature is that it is created by projecting the orbital fluctuations associated with excited states onto the ground state orbital densities. With the PHM defined via a projection operator acting on the particle-hole reduced two-body density matrix [65], the PHM is non-invariant under any unitary transformation into a different orbital basis, as we will show here. Conversely, the TDM is invariant under such transformations, as would be expected for any observable. While this may sound like a shortcoming of the PHM, it can become an asset, as we will discuss.

In principle, we can consider arbitrary unitary transformations, but here we specifically focus on transformations that lead to localized orbitals. In this work we choose the Foster-Boys orbital localization procedure [66] over the Edmiston-Ruedenberg scheme [67], primarily due to the analogous definition of Foster-Boys orbitals to maximally localized Wannier functions in periodic systems [68-70].

Beyond the connection between Foster-Boys orbitals and Wannier functions, lo- 
calized orbitals generally recreate general chemical intuition stemming from Lewis structures, chemical bonds, and the model geometry of the system [68,71]. Likewise, it can also provide clarity as to the electron dynamics expected under excitation, since the chemical bonds of the system are better characterized by the localized orbitals.

We also recall that noninvariance under unitary transformations is a key feature of the self-interaction correction (SIC) approach in DFT [72], and it was recognized early on that an unambiguous implementation of SIC can be achieved with localized orbitals $[73,74]$.

This chapter is organized as follows. In Section 3.2 we will present the formal background: first, we show how the linear-response TDDFT formalism for excitation energies $[75,76]$ behaves under unitary transformation to localized orbitals, and how this affects the TDM and PHM. We will then introduce an approximation called the localized PHM. We then proceed to show how to use simple tools of metric space analysis [77-80] to quantify the differences between canonical and localized PHM. In Section 3.3 we illustrate these concepts using simple one-dimensional (1D) model systems with noninteracting spinless electrons. Section 3.4 gives our conclusions.

\subsection{Formal background}

\subsubsection{Excitation energies with TDDFT: the Casida equation}

The standard approach for calculating excitation energies with TDDFT is via the Casida equation [75], which we briefly summarize here (see [34] for more details). For simplicity, let us consider closed-shell systems that are not spin polarized, and limit 
ourselves to the singlet excitation channel; spin indices are then not needed.

We define the product of Kohn-Sham orbitals as

$$
\Phi_{i a}(\mathbf{r})=\varphi_{i}(\mathbf{r}) \varphi_{a}^{*}(\mathbf{r})
$$

and the difference of Kohn-Sham orbital energies as

$$
\omega_{a i}=\epsilon_{a}-\epsilon_{i} .
$$

Here and in the following, $i, j, k, \ldots$ run over occupied orbitals, and $a, b, c, \ldots$ run over unoccupied orbitals. We assume, mainly for notational simplicity, that the Hartreeexchange-correlation kernel of TDDFT, $f_{\mathrm{Hxc}}$, is frequency-independent, and we define the coupling matrix elements

$$
K_{i a, i^{\prime} a^{\prime}}=2 \int d \mathbf{r} \int d \mathbf{r}^{\prime} \Phi_{i a}^{*}(\mathbf{r}) f_{\mathrm{Hxc}}\left(\mathbf{r}, \mathbf{r}^{\prime}\right) \Phi_{i^{\prime} a^{\prime}}\left(\mathbf{r}^{\prime}\right) .
$$

In terms of these quantities, the Casida equation is

$$
\begin{aligned}
& {\left[\delta_{i i^{\prime}} \delta_{a a^{\prime}} \omega_{a^{\prime} i^{\prime}}+K_{i a, i^{\prime} a^{\prime}}\right] X_{i^{\prime} a^{\prime}}+K_{i a, a^{\prime} i^{\prime}} Y_{i^{\prime} a^{\prime}}=-\Omega X_{i a}} \\
& K_{a i, i^{\prime} a^{\prime}} X_{i^{\prime} a^{\prime}}+\left[\delta_{a a^{\prime}} \delta_{i i^{\prime}} \omega_{a^{\prime} i^{\prime}}+K_{a i, a^{\prime} i^{\prime}}\right] Y_{i^{\prime} a^{\prime}}=\Omega Y_{i a},
\end{aligned}
$$

where here and throughout the chapter, summation over repeated indices is implied.

Equations (3.4) and (3.5) are formally a non-Hermitian eigenvalue problem whose eigenvalues come in pairs, i.e., $\left(-\Omega_{n}, \Omega_{n}\right)$, where $\Omega_{n}$ is the $n$th excitation energy of the interacting system. The associated eigenvectors $X_{i a}^{(n)}, Y_{i a}^{(n)}$ yield the density response 
of the excitation as

$$
\delta n\left(\mathbf{r}, \Omega_{n}\right)=\Phi_{i a}(\mathbf{r}) X_{i a}^{(n)}+\Phi_{i a}^{*}(\mathbf{r}) Y_{i a}^{(n)} .
$$

Furthermore, the eigenvectors $X_{i a}^{(n)}, Y_{i a}^{(n)}$ can be used to construct the oscillator strengths $[34,75]$.

\subsubsection{Transition density matrix and particle-hole map}

Formally, the TDM is defined via the one-particle reduced density matrix operator acting between the many-body ground state and the $n$-th excited state wavefunctions of the system [53]:

$$
\Gamma_{n}^{\text {exact }}\left(\mathbf{r}, \mathbf{r}^{\prime}\right)=\left\langle\Psi_{n}\left|\hat{\rho}\left(\mathbf{r}, \mathbf{r}^{\prime}\right)\right| \Psi_{0}\right\rangle
$$

However, in order to apply the TDM to real-life systems, we must formulate the Kohn-Sham TDM, which is simply a superposition of the TDMs associated with single particle excitations. From the Casida formalism, we obtain

$$
\Gamma_{n}\left(\mathbf{r}, \mathbf{r}^{\prime}\right)=\varphi_{i}(\mathbf{r}) \varphi_{a}^{*}\left(\mathbf{r}^{\prime}\right) X_{i a}^{(n)}+\varphi_{i}^{*}\left(\mathbf{r}^{\prime}\right) \varphi_{a}(\mathbf{r}) Y_{i a}^{(n)}
$$

We then have a function, $\Gamma_{n}\left(\mathbf{r}, \mathbf{r}^{\prime}\right)$, that approximates the many-body TDM, Eq. (3.7), associated with the $n$-th excitation of our system. Importantly, we recover the exact density response of the system when $\mathbf{r}=\mathbf{r}^{\prime}$ :

$$
\Gamma_{n}(\mathbf{r}, \mathbf{r})=\delta n\left(\mathbf{r}, \Omega_{n}\right)
$$

The TDM is not complete, however, as has been discussed previously [62-65]. Notably, the TDM is defined via the one-particle density matrix and only encodes prop- 
erties about particle-hole correlations that can be derived therefrom. If we instead consider the two-body particle-hole density matrix, and then apply a projector onto those excitations, we recover the PHM [65], which contains information about the individual particles and holes created during and excitation. The explicit form of the PHM is

$$
\Xi_{n}\left(\mathbf{r}, \mathbf{r}^{\prime}\right)=\Phi_{i a}\left(\mathbf{r}^{\prime}\right)\left|\varphi_{i}(\mathbf{r})\right|^{2} X_{i a}^{(n)}+\Phi_{i a}\left(\mathbf{r}^{\prime}\right)\left|\varphi_{i}(\mathbf{r})\right|^{2} Y_{i a}^{(n)} .
$$

Equation (3.10) has two important sum rules. First, we can clearly infer from the definition of the PHM that in an orthonormal basis, summation over $\mathbf{r}^{\prime}$ gives zero:

$$
\int d \mathbf{r}^{\prime} \Xi_{n}\left(\mathbf{r}, \mathbf{r}^{\prime}\right)=0
$$

Physically, this result is important, as it guarantees charge conservation in our system.

The second sum rule over $\mathbf{r}$ leaves us with

$$
\int d \mathbf{r} \Xi_{n}\left(\mathbf{r}, \mathbf{r}^{\prime}\right)=\delta n\left(\mathbf{r}^{\prime}, \Omega_{n}\right) .
$$

Importantly, this is the exact density density response associated with the $n$-th excited state of our system.

Usefully, the PHM allows users to graphically interpret that the sum rules are satisfied. By visually summing the red and blue areas along the $\mathbf{r}$ and $\mathbf{r}^{\prime}$ axes (see below), one can quickly make predictions and determine the electronic nature of a given system, making the tool easy to understand and interpret for all readers. 


\subsubsection{Casida equation for localized orbitals}

One noticeable difference between the equations defining the TDM and the PHM is the non-invariance contained within the PHM. Because the TDM is constructed as the expectation value of an operator, the TDM will thus be invariant under any unitary transformation of the orbital basis. The PHM, however, has no similar operator, and thus cannot be described in the same fashion under a transformation.

Let us introduce a set of orbitals $\xi_{\mu}(\mathbf{r})$ which are related to the canonical KohnSham orbital via a unitary transformation:

$$
\varphi_{\nu}(\mathbf{r})=M_{\nu \mu} \xi_{\mu}(\mathbf{r})
$$

(here, the indices $\nu$ and $\mu$ run over all states). We now assume that the unitary transformation matrix $M$ is block diagonal, so that the transformations are restricted to rotations within the occupied and unoccupied subspaces, respectively:

$$
\varphi_{i}(\mathbf{r})=M_{i j}^{\mathrm{o}} \xi_{j}(\mathbf{r}), \quad \varphi_{a}(\mathbf{r})=M_{a b}^{\mathrm{v}} \xi_{b}(\mathbf{r}) .
$$

Here, $M_{i j}^{\mathrm{o}}$ and $M_{a b}^{\mathrm{v}}$ are submatrices of $M$ which operate within the occupied and virtual subspaces.

We define our $\xi_{j}$ and $\xi_{b}$ to be the set of orbitals which are localized according to the Foster-Boys (FB) method; however, any localization procedure could be used in principle, as long as the PHM sum rules are satisfied. That necessitates the requirement that the localization procedure must impose orthonormality on the transformed orbitals. Our choice of localized orbital definition stems from the well known concept that the FB localization method is analogous to Wannier Functions in periodic 
systems. As stated, we chose to optimize our orbitals through the Foster-Boys (FB) method precisely because of this analogue. More explicitly, we chose to transform our orbitals according to the following rotations

$$
\begin{gathered}
I(\varphi)=\sum_{i=1}^{n} \int \varphi_{i}\left(\mathbf{r}_{1}\right) \varphi_{i}\left(\mathbf{r}_{1}\right) \mathbf{r}_{12}^{2} \varphi_{i}\left(\mathbf{r}_{2}\right) \varphi_{i}\left(\mathbf{r}_{2}\right) d^{3} r_{1} d^{3} r_{2} \\
J(\varphi)=\sum_{i>j=1}^{n}\left[\left\langle\varphi_{i}|\overrightarrow{\mathbf{r}}| \varphi_{i}\right\rangle-\left\langle\varphi_{j}|\overrightarrow{\mathbf{r}}| \varphi_{j}\right\rangle\right]^{2} \\
D(\varphi)=\sum_{i=1}^{n}\left[\left\langle\varphi_{i}|\overrightarrow{\mathbf{r}}| \varphi_{i}\right\rangle\right]^{2}
\end{gathered}
$$

All three of these equations are optimized equivalently. Since the final form is the most convenient, that was the equation that was used to transform the canonical orbitals into their localized counterparts; however, the second equation is the most useful to conceptually understand what how the FB method works.

Given a non-interacting system of $n$ particles, we can solve the many-body Schrödinger equation to find the energy eigenstates of the system and their corresponding wavefunctions. Those wavefunctions, since they were solved in the non-interacting limit, are our Kohn-Sham or canonical orbitals. By minimizing $J(\varphi)$ we are maximizing the least-squares distance between the orbital centroids of each state. In other words, we are reducing the orbital overlap between states in real-space, thus localizing our orbitals to a particular region of space. This is equivilent to maximizing $D(\varphi)$, which in practice takes much less time to calculate.

Initially, this seems a mathematical sleight of hand. Since we are simply changing the system by unity, we have done nothing to change the actual physics of the system. In some sense this is true, but LMOs have significant chemical meaning attached 


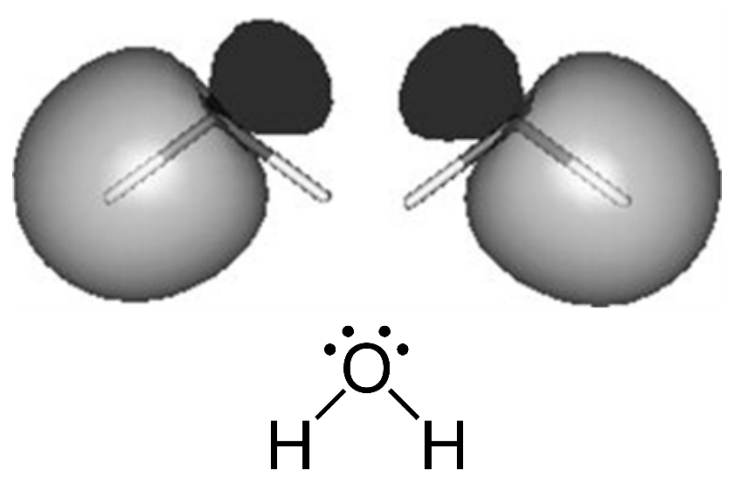

Figure 3.1: (Top) The energy degenerate molecular orbitals for $\mathrm{H}_{2} \mathrm{O}$ calculated by Oña, et al [71]. (Bottom) The Lewis structure for water. Noticeably, the localized orbitals mirror the lewis structure.

to them. While canonical orbitals are generally spread across an entire molecule, localized molecular orbitals tend to follow the description of Lewis structures and interaction sites. This can clearly be seen in Figure 3.1, which shows the calculated localized orbitals, plotted as isosurfaces, compared to the Lewis structure for $\mathrm{H}_{2} \mathrm{O}$ that every introductory chemistry student is familiar with [71].

We will now transform the Casida equation and the resulting TDM and PHM. The first step is to apply our transformation onto the orbital products (3.1):

$$
\Phi_{i a}(\mathbf{r})=M_{i j}^{\mathrm{o}} M_{a b}^{\mathrm{v} *} \Pi_{j b}(\mathbf{r}), \quad \Pi_{j b}(\mathbf{r})=\xi_{j}(\mathbf{r}) \xi_{b}^{*}(\mathbf{r})
$$

The coupling matrix (3.3) thus becomes

$$
K_{i a, i^{\prime} a^{\prime}}=M_{i j}^{\mathrm{o} *} M_{a b}^{\mathrm{v}} M_{i^{\prime} j^{\prime}}^{\mathrm{o}} M_{a^{\prime} b^{\prime}}^{\mathrm{v} *} L_{j b, j^{\prime} b^{\prime}},
$$

where

$$
L_{j b, j^{\prime} b^{\prime}}=2 \int d \mathbf{r} \int d \mathbf{r}^{\prime} \Pi_{j b}^{*}(\mathbf{r}) f_{\mathrm{Hxc}}\left(\mathbf{r}, \mathbf{r}^{\prime}\right) \Pi_{j^{\prime} b^{\prime}}\left(\mathbf{r}^{\prime}\right) .
$$


Substituting this into the Casida equation, Eq. (3.4) and (3.5), we obtain

$$
\begin{aligned}
{\left[\delta_{i i^{\prime}} \delta_{a a^{\prime}} \omega_{a^{\prime} i^{\prime}}+M_{i j}^{\mathrm{o} *} M_{a b}^{\mathrm{v}} M_{i^{\prime} j^{\prime}}^{\mathrm{o}} M_{a^{\prime} b^{\prime}}^{\mathrm{v} *} L_{j b, j^{\prime} b^{\prime}}\right] X_{i^{\prime} a^{\prime}} } & + \\
M_{i j}^{\mathrm{o} *} M_{a b}^{\mathrm{v}} M_{i^{\prime} j^{\prime}}^{\mathrm{o} *} M_{a^{\prime} b^{\prime}}^{\mathrm{v}} L_{j b, b^{\prime} j^{\prime}} Y_{i^{\prime} a^{\prime}} & =-\Omega X_{i a} \\
M_{i j}^{\mathrm{o}} M_{a b}^{\mathrm{v} *} M_{i^{\prime} j^{\prime}}^{\mathrm{o}} M_{a^{\prime} b^{\prime}}^{\mathrm{v}} L_{b j, j^{\prime} b^{\prime}} X_{i^{\prime} a^{\prime}} & + \\
{\left[\delta_{a a^{\prime}} \delta_{i i^{\prime}} \omega_{a^{\prime} i^{\prime}}+M_{i j}^{\mathrm{o}} M_{a b}^{\mathrm{v} *} M_{i^{\prime} j^{\prime}}^{\mathrm{o} *} M_{a^{\prime} b^{\prime}}^{\mathrm{v}} L_{b j, b^{\prime} j^{\prime}}\right] Y_{i^{\prime} a^{\prime}} } & =\Omega Y_{i a} .
\end{aligned}
$$

We can now rearrange some of the summations, and define the transformed eigenvectors $U_{j^{\prime} b^{\prime}}$ and $V_{j^{\prime} b^{\prime}}$ as

$$
U_{j^{\prime} b^{\prime}}=M_{i^{\prime} j^{\prime}}^{\mathrm{o}} M_{a^{\prime} b^{\prime}}^{\mathrm{v}} X_{i^{\prime} a^{\prime}} \quad V_{j^{\prime} b^{\prime}}=M_{i^{\prime} j^{\prime}}^{\mathrm{o}} M_{a^{\prime} b^{\prime}}^{\mathrm{v}} Y_{i^{\prime} a^{\prime}}
$$

Because we have defined $M$ to be a unitary matrix, we can exploit the property $M^{-1}=M^{\dagger}$, and thus obtain the inverse transformation

$$
X_{i^{\prime} a^{\prime}}=M_{i^{\prime} j^{\prime}}^{\mathrm{o}} M_{a^{\prime} b^{\prime}}^{\mathrm{v}} U_{j^{\prime} b^{\prime}} \quad Y_{i^{\prime} a^{\prime}}=M_{i^{\prime} j^{\prime}}^{\mathrm{o}} M_{a^{\prime} b^{\prime}}^{\mathrm{v}} V_{j^{\prime} b^{\prime}}
$$

Substituting this into Eqs. (3.19) and (3.20) gives the transformed Casida equation:

$$
\begin{gathered}
{\left[M_{k i}^{\mathrm{o}} M_{c a}^{\mathrm{v} *} \omega_{c k} M_{k i^{\prime}}^{\mathrm{o} *} M_{c a^{\prime}}^{\mathrm{v}}+L_{i a, i^{\prime} a^{\prime}}\right] U_{i^{\prime} a^{\prime}}+L_{i a, a^{\prime} i^{\prime}} V_{i^{\prime} a^{\prime}}} \\
=-\Omega U_{i a} \\
L_{i a, i^{\prime} a^{\prime}} U_{i^{\prime} a^{\prime}}+\left[M_{k i}^{\mathrm{o}} M_{c a}^{\mathrm{v} *} \omega_{c k} M_{k i^{\prime}}^{\mathrm{o}} M_{c a^{\prime}}^{\mathrm{v} *}+L_{i a, a^{\prime} i^{\prime}}\right] V_{i^{\prime} a^{\prime}} \\
=\Omega V_{i a} .
\end{gathered}
$$


We can see that after inserting our rotated eigenvectors, the system of equations looks largely similar to the original Casida equations. However, a noticeable difference is that the equation elements containing the Kohn-Sham orbital energy differences, $\omega_{a i}$, are no longer diagonal but a full matrix. The difference is subtle, but it clearly makes the eigenvalue equations somewhat more difficult to handle, in addition to mixing the states within our eigenvectors. This was to be expected though, as we were given a warning when we transformed our original orbitals. In that case, the rotation causes a mixing of the canonical orbitals according to the constraints of the localization method being used to find the rotated orbitals, $\xi_{j}$ or $\xi_{b}$.

\subsubsection{Localized PHM and metric}

As stated previously, the TDM is invariant under any unitary transformation. By applying the transformation prescribed in Eqs. (3.14) and (3.22), we can then simply transform the TDM by utilizing the fact that $M$ is unitary. The result is

$$
\Gamma_{n}\left(\mathbf{r}, \mathbf{r}^{\prime}\right)=\xi_{j}(\mathbf{r}) \xi_{b}^{*}\left(\mathbf{r}^{\prime}\right) U_{j b}^{(n)}+\xi_{j}^{*}\left(\mathbf{r}^{\prime}\right) \xi_{b}(\mathbf{r}) V_{j b}^{(n)}
$$

As we can see, we are left with the same mathematical form as Eq. (3.8), with rotated orbitals replacing their canonical counterparts, which implies that the TDM is invariant as expected.

Applying the orbital transformation onto our PHM, and exploiting the unitarity 
of the rotation matrix, we obtain the following expression:

$$
\begin{aligned}
\Xi_{n}\left(\mathbf{r}, \mathbf{r}^{\prime}\right) & =M_{i j}^{\mathrm{o}} M_{i j^{\prime}}^{\mathrm{o} *} \xi_{j}\left(\mathbf{r}^{\prime}\right) \xi_{b}^{*}\left(\mathbf{r}^{\prime}\right) M_{i k}^{\mathrm{o}} M_{i k^{\prime}}^{\mathrm{o} *} \xi_{k}(\mathbf{r}) \xi_{k^{\prime}}^{*}(\mathbf{r}) U_{j^{\prime} b}^{(n)} \\
& +M_{i j}^{\mathrm{o} *} M_{i j^{\prime}}^{\mathrm{o}} \xi_{j}^{*}\left(\mathbf{r}^{\prime}\right) \xi_{b}\left(\mathbf{r}^{\prime}\right) M_{i k}^{\mathrm{o}} M_{i k^{\prime}}^{\mathrm{o} *} \xi_{k}(\mathbf{r}) \xi_{k^{\prime}}^{*}(\mathbf{r}) V_{j^{\prime} b}^{(n)}
\end{aligned}
$$

Comparison with Eq. (3.10) clearly shows that the expression for the PHM is not formally invariant under unitary transformation of the orbitals, as we anticipated. This, of course, is not a problem in and by itself, since the actual values for $\Xi_{n}\left(\mathbf{r}, \mathbf{r}^{\prime}\right)$ can in principle be exactly computed from the knowledge of the eigenvectors of the transformed Casida equation and from the transformation matrices.

As mentioned above, the PHM is derived by projecting excited-states orbital density fluctuations onto ground-state orbital densities. Obviously, the density response of the system will be conserved; however, the amplitude of the rotated states that are being projected onto our ground-state, will change depending on the rotation being applied. With this in mind, we define a new quantity using the same framework of our canonical PHM. By applying our rotation to the canonical orbitals, the localized orbitals can be used to calculate the electron-hole dynamics of a given system, which we term the localized particle-hole map (LPHM):

$$
\Xi_{n}^{\mathrm{loc}}\left(\mathbf{r}, \mathbf{r}^{\prime}\right)=\Pi_{j b}\left(\mathbf{r}^{\prime}\right)\left|\xi_{j}(\mathbf{r})\right|^{2} U_{j b}^{(n)}+\Pi_{j b}^{*}\left(\mathbf{r}^{\prime}\right)\left|\xi_{j}(\mathbf{r})\right|^{2} V_{j b}^{(n)}
$$

It is easy to see that the localized PHM $\Xi_{n}^{\operatorname{loc}}\left(\mathbf{r}, \mathbf{r}^{\prime}\right)$ still satisfies the sum rules (3.10) and (3.11).

It is important that, like the density, the density response is conserved with the 
definition of the LPHM. Additionally, all quantities used to calculate the LPHM can be easily obtained. From the canonical orbitals, $\varphi_{i}(\mathbf{r})$ and $\varphi_{a}(\mathbf{r})$, then using any rotation method that preserves the orthonormality of the orbitals, the Casida eigenvectors can be calculated. Preserving orthonormality is a key caveat of the LPHM, as some localization procedures only keep orbitals pseudo-orthonormalized.

The LPHM is another tool in the toolbox to evaluate and visualize electronic excitations. Used in concert with the TDM and the canonical PHM, the LPHM offers a complementary method that can help decipher the electronic dynamics in a system. To that end, it is well known that the Foster-Boys localization method is analogous to Wannier Functions in periodic systems. While the TDM offers no difference in viewpoint of these two environments, the PHM and LPHM could potentially scrub away at some of the electron dynamics within a periodic system and provide additional insight into real-space electron analysis. Our simple 1D examples will shed some light on this notion.

We can go further and quantify the differences between the canonical PHM, $\Xi_{n}$, and the LPHM, $\Xi_{n}^{\text {loc }}$, using an appropriate metric. We will use the definition of the metric space developed by D'Amico and coworkers [77-80]. To be specific, we define

$$
D_{\Xi}=\int d \mathbf{r} \int d \mathbf{r}^{\prime}\left|\Xi_{n}\left(\mathbf{r}, \mathbf{r}^{\prime}\right)-\Xi_{n}^{\mathrm{loc}}\left(\mathbf{r}, \mathbf{r}^{\prime}\right)\right|
$$

In essence, we thus take the vector norm between the canonical and the localized PHM. It is convenient to have a metric that lives in the interval $[0,1]$, so we redefine

$$
\tilde{D}_{\Xi}=\frac{D_{\Xi}}{\int d \mathbf{r} \int d \mathbf{r}^{\prime}\left\{\left|\Xi_{n}\left(\mathbf{r}, \mathbf{r}^{\prime}\right)\right|+\left|\Xi_{n}^{\text {loc }}\left(\mathbf{r}, \mathbf{r}^{\prime}\right)\right|\right\}} .
$$


It can easily be seen that $\tilde{D}_{\Xi}$ lies on our desired interval through triangular inequality.

As we will see below, $\tilde{D}$ is a useful descriptor for the excitation processes we are interested in, but we will also employ another, more direct way to quantify the impact of localization. Having defined our rotation matrices in Eq. (3.14), we can clearly see that in the case of no orbital rotation the rotation matrices $M^{\mathrm{o}, \mathrm{v}}$ are simply the identity. Using this, we can quantify how much our localization procedure transforms the orbitals. We define the localization number $\bar{M}$ as

$$
\bar{M}=\frac{1}{N_{\mathrm{occ}} N_{\mathrm{virt}}}\left\|M_{i j}^{\mathrm{o}}-\delta_{i j}\right\|\left\|M_{a b}^{\mathrm{v}}-\delta_{a b}\right\|
$$

where $\|M\|$ is the Frobenius norm of the matrix $M$ and $N_{\text {occ }}$ and $N_{\text {virt }}$ are the number of occupied and virtual states being localized in the calculation. $\bar{M}$ is a measure for how much the canonical orbitals are being localized.

However, not all orbitals are relevant for a particular excitation, and, in fact, an excitation is usually dominated by one or few Kohn-Sham orbital transitions $i \rightarrow a$. To quantify this in a simple descriptor, we define the transition localization number:

$$
\bar{M}_{t}=\sum_{i a}\left(\left|X_{i a}\right|+\left|Y_{i a}\right|\right) \frac{\sqrt{\sum_{j}\left|M_{i j}^{\mathrm{o}}-\delta_{i j}\right|^{2} \sum_{b}\left|M_{a b}^{\mathrm{v}}-\delta_{a b}\right|^{2}}}{N_{\mathrm{occ}} N_{\mathrm{virt}}}
$$

where the summations are explicitly indicated for clarity. The quantity $\bar{M}_{t}$ measures the extent to which the Kohn-Sham transition densities are affected by the localization.

Upon calculating $\bar{M}, \bar{M}_{t}$ and $\tilde{D}_{\Xi}$, we can use these to analyze and quantify the effects of localization for various types of systems. We expect that the differences will be largest in systems where the excitations have a strong charge-transfer charac- 

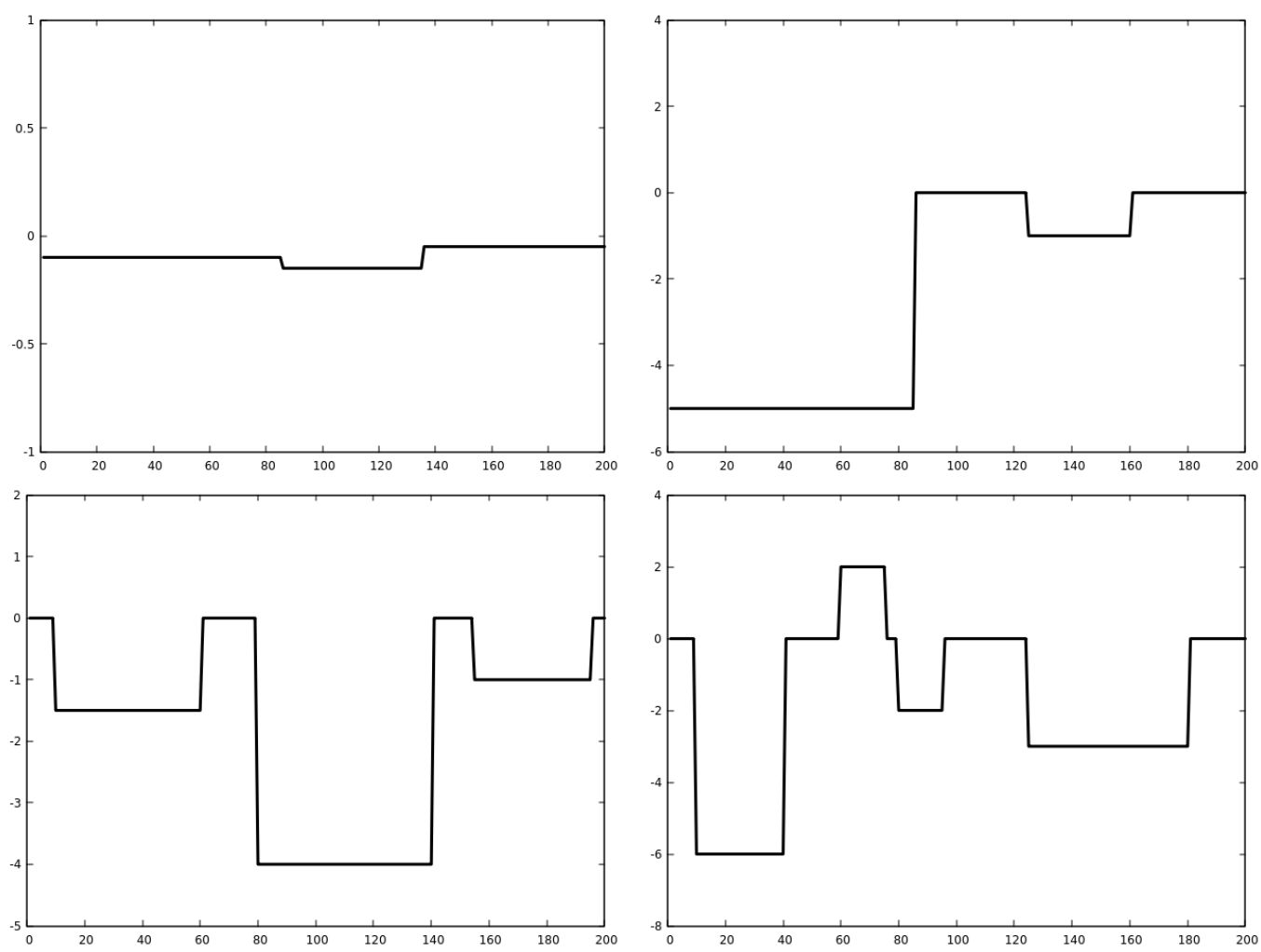

Figure 3.2: The potentials used for the calculations are a single well system (a), a double well system (b), and two triple well systems, labeled as A and B in Table 3.1, respectively (c and $\mathrm{d}$ ).

ter. We anticipate that the metric analysis tool will allow us to define a "region of usefulness", where the differences are neither too small nor too large.

\subsection{Results}

Having formulated and defined both the LPHM and our metric to quantify the differences between the canonical and localized cases, we can use simple 1D systems to demonstrate the utility of our methods. Figure 3.2 shows four different potentials, each of them defined on a 200-point numerical grid with hard-wall boundaries. We 


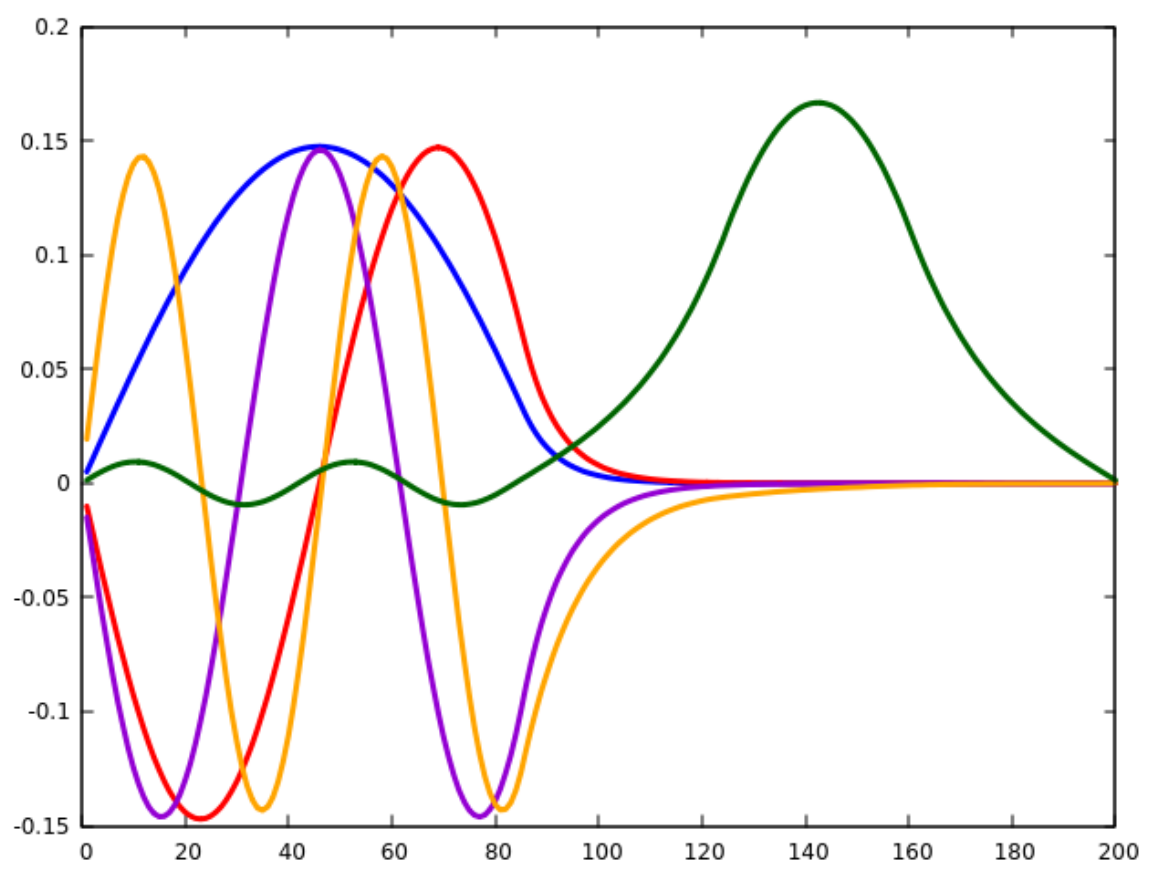

Figure 3.3: Canonical orbitals for the double well potential, see Fig. 3.2b. Four of the five occupied states are in the deep well, while only one state occupies the shallow right well. States are colored as follows: Lowest - blue, 2 - red, 3 - purple, 4 - orange, highest - green.
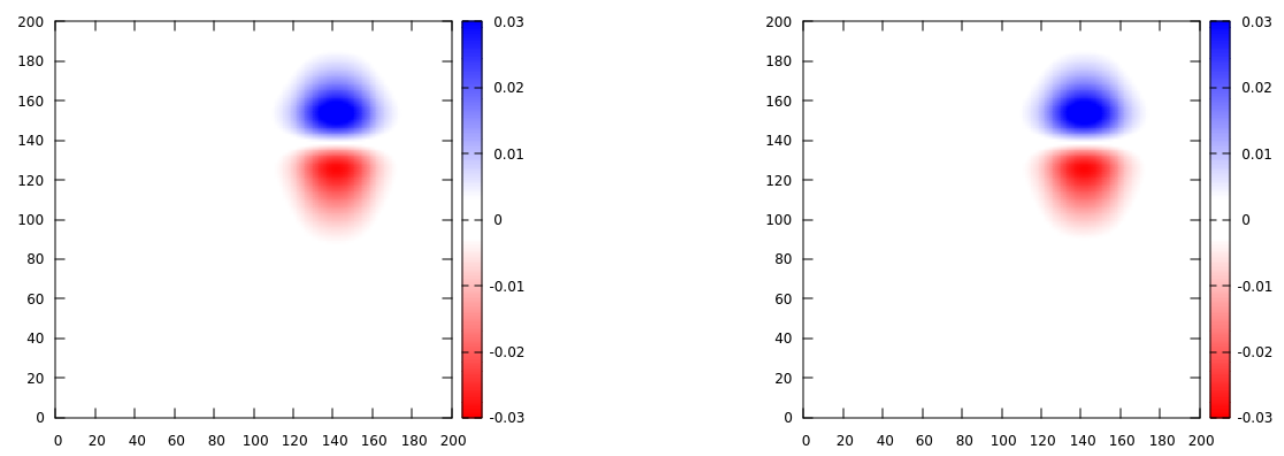

Figure 3.4: The canonical (top) and localized PHM (bottom) for a deep double well potential (Fig. 3.2b). The two PHMs are nearly identical, primarily due to the localized nature of the canonical orbitals prior to applying the localization procedure. 


\begin{tabular}{lccc}
\hline System & $\bar{M}$ & $\bar{M}_{t}$ & $\tilde{D}_{\Xi}$ \\
\hline Single Well & 0.553 & 0.230 & 0.583 \\
Double Well & 0.570 & $8.92 \times 10^{-3}$ & $2.91 \times 10^{-4}$ \\
Triple Well A & 0.685 & 0.252 & 0.921 \\
Triple Well B & 0.508 & 0.196 & 0.507 \\
Triple Well B & 0.508 & 0.197 & 0.967 \\
\hline
\end{tabular}

Table 3.1: Various descriptors for the PHMs for different excitations: localization number $\bar{M}$, Eq. (3.30), transition localization number $\bar{M}_{t}$, Eq. (3.31), and PHM metric $\tilde{D}_{\Xi}$, Eq. (3.29). $\mathrm{B}_{1}$ and $\mathrm{B}_{4}$ stands for the first and the fourth excitation of the Triple Well B system, respectively.

solve the 1D noninteracting Schrödinger equation in each case, where it is implicitly assumed that the given potential is the Kohn-Sham potential. We then solve the Casida equation to obtain the excitation energies and eigenvectors, using the randomphase approximation (RPA), i.e., only the Hartree kernel, with a soft-Coulomb potential [63]. The occupied orbitals are then localized using the Foster-Boys method, and the PHMs are computed. For simplicity, we did not localize the virtual orbitals, but this does not affect our conclusions and requires only trivial modifications of the formulas for the metric and localization numbers.

We begin with a deep two-well system populated with 10 electrons. There is no spin-dependence, so we calculate five orbitals that are assumed to be doubly occupied by spin up and spin down electrons. As shown in Fig. 3.2b, the wells are deep (the left one more so than the right one) and well separated from one another. Because of the depth and separation of the wells, the orbitals for the canonical case are well localized prior to applying any localization procedure onto the orbitals. Additionally, we can see in Fig. 3.3 that the highest occupied orbital in this case is the only state located in the right, shallower well.

The associated canonical and localized PHMs are plotted in Fig. 3.4, for the 

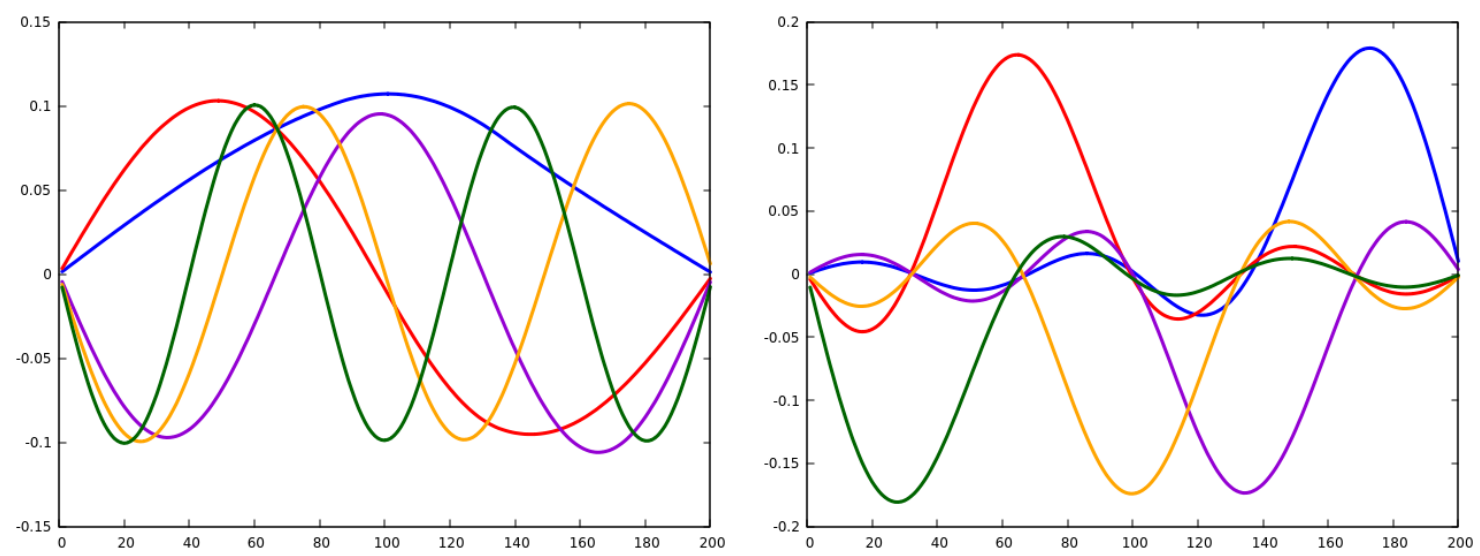

Figure 3.5: The lowest five canonical orbitals (top) and localized orbitals (bottom) for the single well system with ten spin-independent electrons. Lowest state - blue, 2 - red, 3 - purple, 4 - orange, highest - green.

lowest excitation. In our earlier work [63-65] we had explained in detail how to read PHMs: the horizontal coordinate indicates where electrons or holes are coming from, and the vertical coordinate tells us where they are going to. Blue and red pixels in the resulting map indicate electrons and holes, respectively. Thus, we see a highly localized excitation in Fig. 3.4, with charge staying in the same spatial region. Employing Eqs. (3.28) and (3.29), we get a very small $\tilde{D}_{\Xi}$ value on the order of $10^{-4}$, see Table 3.1. The localization number, $\bar{M}$, is quite high, as this includes the mixing of the lowest 4 states which, however, do not contribute to this excitation. On the other hand, the transition localization number, $\bar{M}_{t}$, is very small, which shows that this excitation involves canonical states that are already strongly localized. Consistent with the values of these descriptors, there is nearly no discernible difference between the LPHM and PHM in Fig. 3.4. Again, this is not surprising: the relevant states for this particular excitation in this system were already localized before the localization procedure was employed.

Going further, we now look at systems where the localization has a more profound 

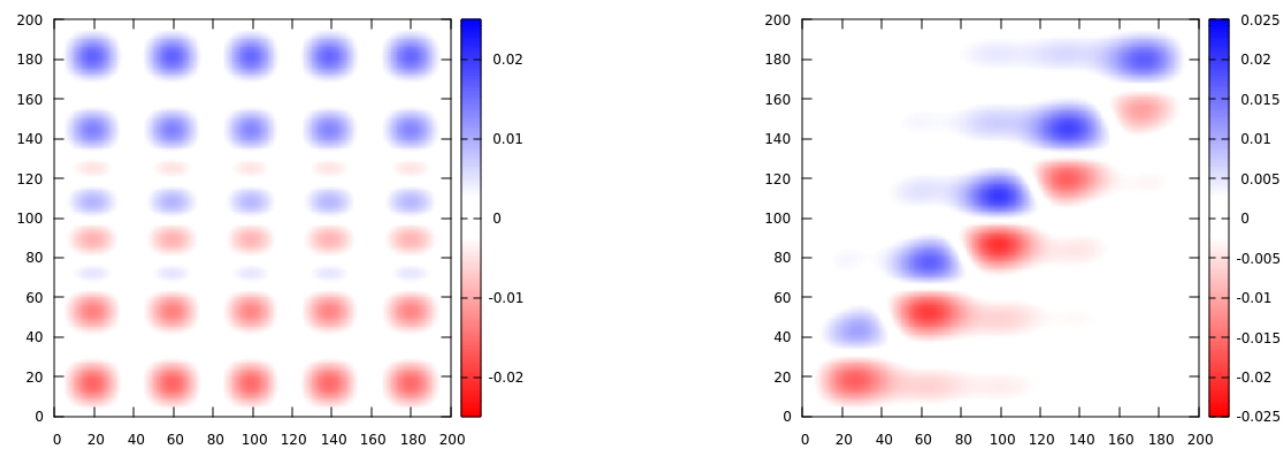

Figure 3.6: The canonical PHM (top) and the LPHM (bottom) for the lowest excitation of the single well system, with 5 doubly occupied orbitals.

effect. Our first case is the single well system depicted in Fig. 3.2a. We begin by looking at the effect of localization on the orbitals, which can be seen in Fig. 3.5. Very clearly, we can see the effect of the localization, shifting the orbitals to avoid overlapping with one another as much as is possible in a $1 \mathrm{D}$ system. Since the well is relatively shallow, these changes occur across the entire well. As we can see from the localization numbers for this system given in Table 3.1, both localization numbers are sizable, and so is $D_{\Xi}$ for the excitation under consideration. In accordance with these numbers, we can see in Fig. 3.6 that there is a large difference between the PHM and LPHM. From the PHM, we can see a very diffuse general density response that distributes electrons and holes from all regions of the well. The LPHM, on the other hand, suggests a response that proceeds in steps between neighboring regions, eliminating any long-range charge transfer.

Now, both of the previous excitations demonstrate two different processes. The double well demonstrated a localized excitation, giving us essentially identical canonical and localized PHMs. The shallow single well, however, was a system that demon- 
strated a largely delocalized excitation behavior where electrons and holes do not have distinctly localized origins. The LPHM tends to collapse this excitation into shortrange "hops", which clearly contradicts the picture given by the canonical PHM.

For the final examples, we want to look at systems where our states show some localization, but still have a very specific, delocalized excitation character. To do this, we use two triple well systems, shown in Figs. 3.2c and d.

We begin with the Triple Well B system, where we compare the first and the fourth excitations. $\bar{M}$ and $\bar{M}_{t}$ are similar in both cases, indicating moderate amounts of orbital localization. The top panels of Figs. 3.7 and 3.8 suggest that both excitations are largely localized, primarily being the exchange of holes and electrons between the plateaus adjacent to the small middle well. The PHM of the first excitation is more faint than that of the fourth one, suggesting a larger oscillator strength of the latter. Interestingly, this is reflected in the metric $D_{\Xi}$, which is almost twice as large for the fourth excitation, which shows that the metric is a rather sensitive indicator of subtle differences between PHM and LPHM that would be difficult to discern by mere inspection.

For both excitations of the Triple Well B, the LPHM shifts the emphasis from the two central plateaus, involving the potential well in the middle. The LPHM suggests that the middle well emits holes to the left plateau and electrons to the right one, which seems more in accordance with physical intuition than what is displayed in the canonical PHM. However, some of the charge-transfer features of the canonical PHM are preserved in the LPHM, although there is clearly a stronger emphasis on the diagonal region of the map.

Finally, in the Triple Well A system, we analyze a clear case of charge-transfer. In 

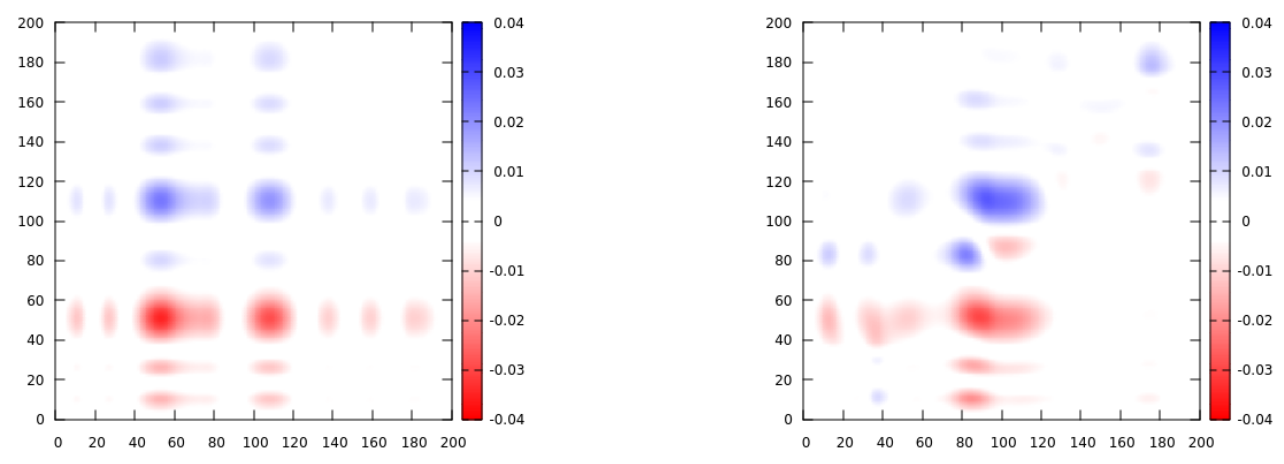

Figure 3.7: (The canonical PHM (top) and the LPHM (bottom) of the first excitation in the Triple Well B system.
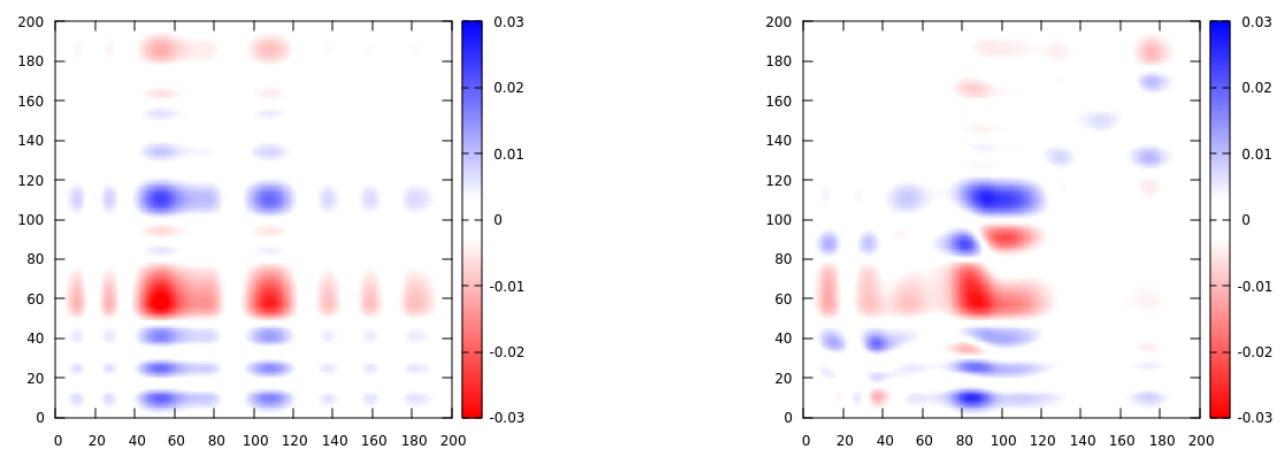

Figure 3.8: The canonical PHM (top) and the LPHM (bottom) of the fourth excitation in the Triple Well B system. 
Fig. 3.9, we can see a large transfer of electron density from the rightmost well, across the center well, and ending in the leftmost well. Largely the same characteristics are found in the LPHM, although a bit more emphasis is placed on the diagonal (these features are too faint to be seen in the canonical PHM). Thus, the charge transfer characteristic of the system is preserved upon localization, despite reducing the orbital overlap and, hence, a rather large value of $\tilde{D}_{\Xi}$.

There is additional information, such as the subtle redistribution of holes and electrons through the central well, that are potentially interesting for three-dimensional (3D) systems. For example, one could imagine a system where a charge transfer is mediated by some functional unit. Clearly, one could capture the charge transfer across the system through the PHM or TDM, but the LPHM could provide additional insight into the role the functional unit plays in mediating the charge transfer across the molecule. Thus, the localization procedure could identify functional units that play a large role in charge transfer. For example, many organic polymers and molecules, which are commonly used in organic photovoltaics and other organic electronics, will have delocalized canonical orbitals that are essentially created from the $p_{z}$ orbitals in the molecule, but some systems, notably those with intramolecular charge-transfer character, will have sizable degrees of localization already at the level of canonical orbitals. These features can be made more explicit when the LPHM is used.

\subsection{Conclusions}

In this chapter, we have investigated the effects of orbital localization on visualizing excitations with the PHM. As we have demonstrated with four different 1D model 

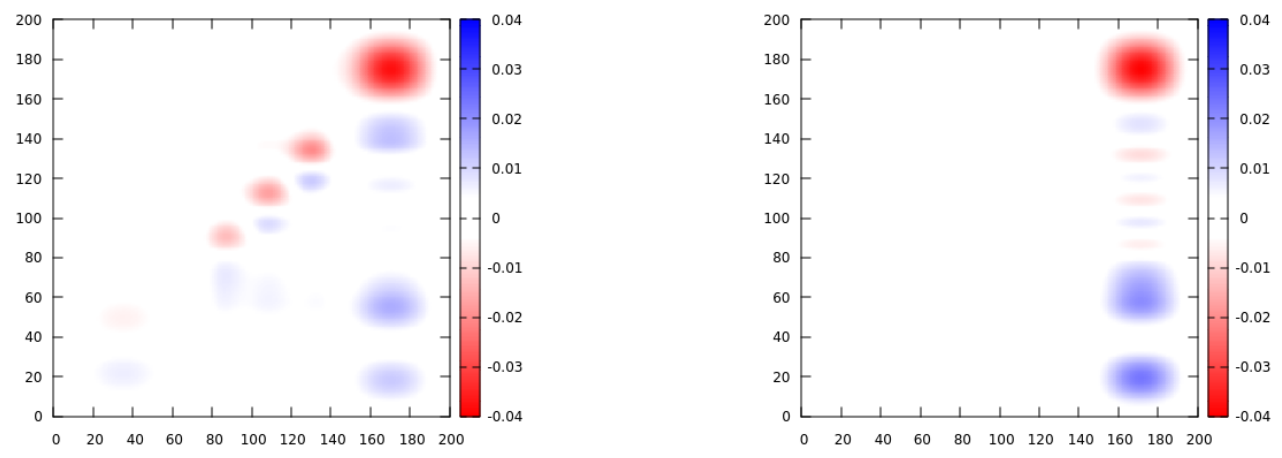

Figure 3.9: The canonical PHM (top) and the LPHM (bottom) of the first excitation in the Triple Well A system.

systems, the localization of each system can be characterized quantitatively in two ways. First, we can calculate localization numbers (defined via the orbital rotation matrices), thus quantifying the changes to the relevant orbitals. Second, using the canonical PHM and the LPHM in tandem, we can calculate an appropriate metric that describes the character of the system. Combining those descriptors, we are able to quantitatively categorize systems and discover if any discernible information can be gained from the LPHM. However, as the example with the shallow single well has taught us, one should not overly rely on the descriptors alone: if the system is very delocalized to begin with, the LPHM tends to overshoot and suggest a collapse of any long-range charge transfer. Whether this is physical or not remains to be further investigated, for instance by comparison with accurate benchmark calculations.

On the other hand, for systems and excitations that have a mixed localizeddelocalized character to begin with, the LPHM tends to enhance and sharpen the role of individual functional units. This clearly helps in identifying and visualizing the underlying excitation mechanisms. 
While this study did not rigorously test the limits of our metric, our 1D examples at least suggest that the LPHM used in tandem with our quantitative schemes can help gain useful insight into molecular excitation dynamics. High values of the metric, as demonstrated in the Triple Well A system, appear to be be interesting for examining charge transfer systems: here, the LPHM performs well, providing an indicator of the robustness of the charge transfer character. A main reason for this is that the LPHM is constrained by sum rules, and gives the exact same density response as in the canonical system.

Further testing must be undertaken to refine these predictions. The 1D systems studied here are hampered by the unrealistic hard well walls at the boundaries of the numerical grid; work is currently in progress towards realizing a 3D version of the LPHM for real molecular systems. Another fruitful direction of study would be to apply the LPHM to excitonic effects in periodic solids. 


\section{Chapter 4}

\section{Exchange-correlation torque effects: exact comparison via the Hubbard Lattice}

As a disclaimer, much of this chapter has been written verbatim and readapted from the work and supplemental materials in E. A. Pluhar and C. A. Ullrich, Phys. Rev. $B$ 100, 125135 (2019) [81].

\subsection{Introduction}

Spin-density-functional theory (SDFT) [82-84] is an in principle exact framework for systems of $N$ interacting electrons subject to the many-body Hamiltonian

$$
\hat{H}=\sum_{j}^{N}\left[-\frac{\nabla_{j}^{2}}{2}+V\left(\mathbf{r}_{j}\right)+\boldsymbol{\sigma}_{j} \cdot \mathbf{B}\left(\mathbf{r}_{j}\right)\right]+\frac{1}{2} \sum_{j \neq k}^{N} \frac{1}{\left|\mathbf{r}_{j}-\mathbf{r}_{k}\right|} .
$$


Here, $\boldsymbol{\sigma}_{j}$ is the vector of Pauli matrices acting on the spin of the $j$ th electron, $V(\mathbf{r})$ is an external scalar potential, and $\mathbf{B}(\mathbf{r})$ is an external magnetic field which can be noncollinear, i.e., whose magnitude and direction can vary in space. The Bohr magneton, $\mu_{B}=e \hbar / 2 m$, has been absorbed in the definition of the magnetic field, and we use atomic units $\left(e=m=\hbar=4 \pi \epsilon_{0}=1\right)$ throughout.

The corresponding noninteracting system in SDFT obeys the Kohn-Sham equation

$$
\left[\left(-\frac{\nabla^{2}}{2}+V_{\mathrm{KS}}(\mathbf{r})\right) I+\boldsymbol{\sigma} \cdot \mathbf{B}_{\mathrm{KS}}(\mathbf{r})\right] \Psi_{i}(\mathbf{r})=\epsilon_{i} \Psi_{i}(\mathbf{r}),
$$

where $I$ is the $2 \times 2$ unit matrix and the Kohn-Sham orbitals $\Psi_{i}(\mathbf{r})$ are two-component spinors. The scalar Kohn-Sham potential is the sum of external, Hartree, and exchangecorrelation (xc) potentials, $V_{\mathrm{KS}}=V+V_{\mathrm{H}}+V_{\mathrm{xc}}$, and the Kohn-Sham magnetic field contains external and xc contributions, $\mathbf{B}_{\mathrm{KS}}=\mathbf{B}+\mathbf{B}_{\mathrm{xc}}$. In general, $\mathbf{B}_{\mathrm{xc}}$ can be nonvanishing even if $\mathbf{B}=0$, i.e., for open-shell or spontaneously magnetized systems.

The effects of $\mathbf{B}_{\mathrm{xc}}$ are present in a wide variety of systems, including materials with noncollinear magnetism, canted or spiral magnetization, or frustrated spins [85-87]. In these examples, spin-orbit coupling often plays an important role. The SDFT formalism can be generalized to include spin-orbit coupling $[87,88]$; in this chapter we ignore spin-orbit coupling to keep the discussion simple.

Formally, $V_{\mathrm{xc}}$ and $\mathbf{B}_{\mathrm{xc}}$ are defined as functionals of the electronic density $n(\mathbf{r})=$ $\sum_{i}^{N} \operatorname{tr}\left[\Psi_{i}(\mathbf{r}) \Psi_{i}^{\dagger}(\mathbf{r})\right]$ and the magnetization [89] $\mathbf{m}(\mathbf{r})=\sum_{i}^{N} \operatorname{tr}\left[\boldsymbol{\sigma} \Psi_{i}(\mathbf{r}) \Psi_{i}^{\dagger}(\mathbf{r})\right]$; in practice, $V_{\mathrm{xc}}$ and $\mathbf{B}_{\mathrm{xc}}$ need to be approximated. Almost all approximations used in SDFT for noncollinear magnetism are based on common local and semilocal xc functionals such as the local spin-density approximation, LSDA $[13,90]$, or the generalized gradient approximations, GGAs $[18,91]$. The standard implementation of LSDA or GGAs 
assumes a local spin quantization axis which is aligned with the local $\mathbf{m}(\mathbf{r})[92,93]$; this produces a $\mathbf{B}_{\mathrm{xc}}(\mathbf{r})$ that is parallel to $\mathbf{m}(\mathbf{r})$ everywhere.

In general, $\mathbf{B}_{\mathrm{xc}}(\mathbf{r})$ can have components perpendicular to $\mathbf{m}(\mathbf{r})$, which give rise to local xc torques,

$$
\tau_{\mathrm{xc}}(\mathbf{r})=\mathbf{m}(\mathbf{r}) \times \mathbf{B}_{\mathrm{xc}}(\mathbf{r})
$$

The zero-torque theorem [94] mandates that

$$
\int d \mathbf{r} \tau_{\mathrm{xc}}(\mathbf{r})=0
$$

since a system cannot exert a net torque on itself. For the LSDA and GGAs, the local $\mathrm{xc}$ torque is zero everywhere, so the zero-torque theorem is trivially satisfied. As we will see, other approximations may not satisfy the zero-torque theorem a priori, but there are ways in which it can be enforced.

Several approximations for $\mathbf{B}_{\mathrm{xc}}$ that include xc torque effects have been proposed in the literature, based on the spin-spiral state of the electron gas [95-98], modified GGAs [99-102], the exchange-only optimized effective potential (OEP) [103], or a source-free construction [104]. These methods were applied to a variety of noncollinear spin systems; however, a rigorous assessment has been hampered by the absence of exact or at least highly accurate reference calculations.

In this chapter, we perform benchmark studies for a simple model system, namely, two electrons on a linear 4-point extended Hubbard lattice (1/4 filled Hubbard tetramer) with noncollinear magnetic fields. In Section 4.2 we will give the details of the model, and explain why it is an appropriate choice for studying xc torques.

Results will be presented for two scenarios: a lattice Hamiltonian without any 
symmetries (Sec. 4.3), and a Hamiltonian with $C_{2}$ symmetry (Sec. 4.4). In both cases, we obtain the exact ground-state energy, densities, and magnetizations from the exact solutions, and compare with approximate Kohn-Sham results [105,106]. We reverseengineer the exact xc potentials and magnetic fields for interaction strengths from the weakly to the strongly correlated regime, and discuss how the Kohn-Sham system mimics the behavior of the many-body system as the electrons begin to localize. We summarize our results in Section 4.5 and conclude with a general outlook.

\subsection{SDFT for the extended Hubbard model with noncollinear magnetic fields}

We consider P-point Hubbard chains with on-site (o.s.) and nearest-neighbor (n.n.) interactions and with external scalar potentials and magnetic fields, $V_{k}$ and $\mathbf{B}_{k}$, where $k$ denotes the lattice sites. The continuum Hamiltonian (4.1) is thus replaced by the extended Hubbard model Hamiltonian [107-110]

$$
\begin{gathered}
\hat{H}_{\text {lat }}=-t \sum_{\langle k, l\rangle \sigma}\left(\hat{c}_{k \sigma}^{\dagger} \hat{c}_{l \sigma}+\hat{c}_{l \sigma}^{\dagger} \hat{c}_{k \sigma}\right)+U_{0} \sum_{k} \hat{c}_{k \uparrow}^{\dagger} \hat{c}_{k \uparrow} \hat{c}_{k \downarrow}^{\dagger} \hat{c}_{k \downarrow} \\
+U_{1} \sum_{\langle k, l\rangle}\left(\hat{\mathbf{c}}_{k}^{\dagger} \hat{\mathbf{c}}_{k}\right)\left(\hat{\mathbf{c}}_{l}^{\dagger} \hat{\mathbf{c}}_{l}\right)+\sum_{k}\left[V_{k} \hat{\mathbf{c}}_{k}^{\dagger} \hat{\mathbf{c}}_{k}+\mathbf{B}_{k} \cdot \hat{\mathbf{c}}_{k}^{\dagger} \boldsymbol{\sigma} \hat{\mathbf{c}}_{k}\right] .
\end{gathered}
$$

Here, $\hat{c}_{k \sigma}^{\dagger}$ and $\hat{c}_{k \sigma}$ are creation and annihilation operators for electrons with spin $\sigma=\uparrow, \downarrow$ on site $k$, and $\hat{\mathbf{c}}_{k}^{\dagger}$ and $\hat{\mathbf{c}}_{k}$ are these operators arranged as two-component row and column vectors. $\langle k, l\rangle$ denotes nearest-neighbor lattice sites. We fix the hopping parameter as $t=0.5$, following Carrascal et al. [111]. The o.s. interaction strength $U_{0}$ will be varied, and the n.n. interaction strength will be taken as $U_{1}=U_{0} / 2$, which 
is typical for many materials of interest [112].

We obtain the two-electron ground state of $\hat{H}_{\text {lat }}$, using nonperiodic boundary conditions, by diagonalization within a complete basis of $P(P+1) / 2$ singlet states and $3 P(P-1) / 2$ triplet states, which yields the exact ground-state energy $E_{\mathrm{gs}}$ and the exact ground-state density and magnetization, $n_{k}$ and $\mathbf{m}_{k}$, on each lattice site.

The extended Hubbard model described above can be treated using SDFT [113]; the corresponding Kohn-Sham lattice Hamiltonian follows from $\hat{H}_{\text {lat }}$ by setting $U_{0}=$ $U_{1}=0$ and replacing $V_{k}$ and $\mathbf{B}_{k}$ by the Kohn-Sham scalar potential and magnetic field $V_{\mathrm{KS}, k}$ and $\mathbf{B}_{\mathrm{KS}, k}$, respectively. We invert the lattice analog of the Kohn-Sham equation, Eq. (4.2), to obtain those $V_{\mathrm{KS}, k}$ and $\mathbf{B}_{\mathrm{KS}, k}$ which reproduce the exact $n_{k}$ and $\mathbf{m}_{k}$ on the $P$-point lattice. Since the external potential and magnetic field are given, and the Hartree potential is easily obtained as

$$
V_{\mathrm{H}, k}=U_{0} n_{k}+U_{1}\left(n_{k-1}+n_{k+1}\right)
$$

(defining $n_{0}=n_{P+1}=0$ to account for the nonperiodic boundary conditions), this immediately yields the exact $V_{\mathrm{xc}, k}$ and $\mathbf{B}_{\mathrm{xc}, k}$. There exist a variety of algorithms to invert Kohn-Sham equations [114]; here, we use a conjugate-gradient minimization.

\subsubsection{Inverting the Kohn-Sham equation in noncollinear spin- DFT}

The inverse problem in Kohn-Sham density-functional theory (KS DFT) can be stated as follows: what is the local effective potential which generates a given density as the ground-state density of a KS system? Often, the given density is the exact (or highly 
accurate) density of a many-body system in a prescribed external potential, which was obtained by other means (such as highly accurate and expensive many-body techniques); by inversion, one can then construct the exact exchange-correlation (xc) potential associated with this density. The exact xc potential can then be compared with approximations. There are various numerical techniques to reverse-engineer the KS potential for a given density, as reviewed by Jensen and Wasserman [114].

Here, the task is to construct that KS system which reproduces a target 4-density $\vec{m}_{k}^{t}, k=1, \ldots, P$, on a $P$-site Hubbard chain, where the 4 -vector notation $\vec{m}_{k}^{t}=$ $\left(n_{k}, \mathbf{m}_{k}\right)$ means that the zeroth component is the scalar density (or site occupation), followed by the three components of the magnetization on site $k$. This vector notation was introduced in Ref. [105].

The KS system which reproduces $\vec{m}_{k}^{(t)}$ is characterized by a 4-potential $\vec{v}_{\mathrm{KS}, k}=$ $\left(V_{\mathrm{KS}, k}, \mathbf{B}_{\mathrm{KS}, k}\right), k=1, \ldots, P$, where the zeroth component is the KS scalar potential and the remaining three components are those of the KS magnetic field [105].

Once the target 4-density $\vec{m}_{k}^{t}$ has been calculated via exact diagonalization of the interacting Hamiltonian, we define an iterative procedure to construct $\vec{v}_{\mathrm{KS}, k}$ by means of a cost functional $F\left[\vec{v}_{\mathrm{KS}}^{(i)}\right]$, where the superscript denotes the $i$ th iteration step:

$$
F\left[\vec{v}_{\mathrm{KS}}^{(i)}\right]=\sum_{k=1}^{P}\left|\vec{m}_{k}^{(i)}\left[\vec{v}_{\mathrm{KS}}^{(i)}\right]-\vec{m}_{k}^{t}\right|^{2}
$$

This cost functional is positive definite, and, thanks to the Hohenberg-Kohn theorem, there is a single, unique minimum that occurs when $\vec{m}_{k}^{(i)}$, the density configuration in the $i$ th iteration step, agrees with the target density $\vec{m}_{k}^{t}$. At the minimum, the cost functional has the value zero. In practice, the search is declared a success when the value of $F$ falls below an appropriately chosen small threshold. 
To implement a conjugate gradient (CG) search algorithm [115], we need the derivative of the cost functional:

$$
\frac{\partial F}{\partial v_{\mathrm{KS}, j q}^{(i)}}=\sum_{k=1}^{P} 2\left(\vec{m}_{k}^{(i)}-\vec{m}_{k}^{t}\right) \cdot \frac{\partial \vec{m}_{k}^{(i)}}{\partial v_{\mathrm{KS}, j q}^{(i)}}
$$

where the index $j$ runs over all $P$ lattice sites, and the index $q=0,1,2,3$ runs over the 4 components of $\vec{v}_{\mathrm{KS}, j}^{(i)}$. We have

$$
\vec{m}=\left(\begin{array}{c}
m_{0} \\
m_{1} \\
m_{2} \\
m_{3}
\end{array}\right)=\left(\begin{array}{c}
n_{\uparrow \uparrow}+n_{\downarrow \downarrow} \\
n_{\uparrow \downarrow}+n_{\downarrow \uparrow} \\
i\left(n_{\uparrow \downarrow}-n_{\downarrow \uparrow}\right) \\
n_{\uparrow \uparrow}-n_{\downarrow \downarrow}
\end{array}\right),
$$

where the KS spin-density matrix follows by summing over the occupied KS orbitals:

$$
\left(\begin{array}{cc}
n_{\uparrow \uparrow} & n_{\uparrow \downarrow} \\
n_{\downarrow \uparrow} & n_{\downarrow \downarrow}
\end{array}\right)=\sum_{a=1}^{\text {occ }}\left(\begin{array}{cc}
\left|\psi_{a \uparrow}\right|^{2} & \psi_{a \uparrow} \psi_{a \downarrow}^{*} \\
\psi_{a \downarrow} \psi_{a \uparrow}^{*} & \left|\psi_{a \downarrow}\right|^{2}
\end{array}\right)
$$

Hence, to evaluate $\partial \vec{m}_{k}^{(i)} / \partial v_{\mathrm{KS}, j q}^{(i)}$ in Eq. (4.8), we need derivatives of the KS spin orbitals with respect to $v_{\mathrm{KS}, j b}^{(i)}$. These can be obtained from first-order perturbation theory [dropping the superscript $(i)]$ :

$$
\frac{\partial \psi_{a \sigma, k}}{\partial v_{\mathrm{KS}, j q, k^{\prime}}}=\sum_{b \neq a} \frac{\Psi_{b, k^{\prime}}^{\dagger} \sigma_{q} \Psi_{a, k^{\prime}}}{\epsilon_{a}-\epsilon_{b}} \psi_{b \sigma, k}
$$

where $\epsilon_{a}$ and $\epsilon_{b}$ are KS energy eigenvalues, and $\Psi_{b, k^{\prime}}^{\dagger}=\left(\psi_{b \uparrow, k^{\prime}}^{*}, \psi_{b \downarrow, k^{\prime}}^{*}\right)$.

The ingredients of the CG algorithm to minimize the cost function are now all 


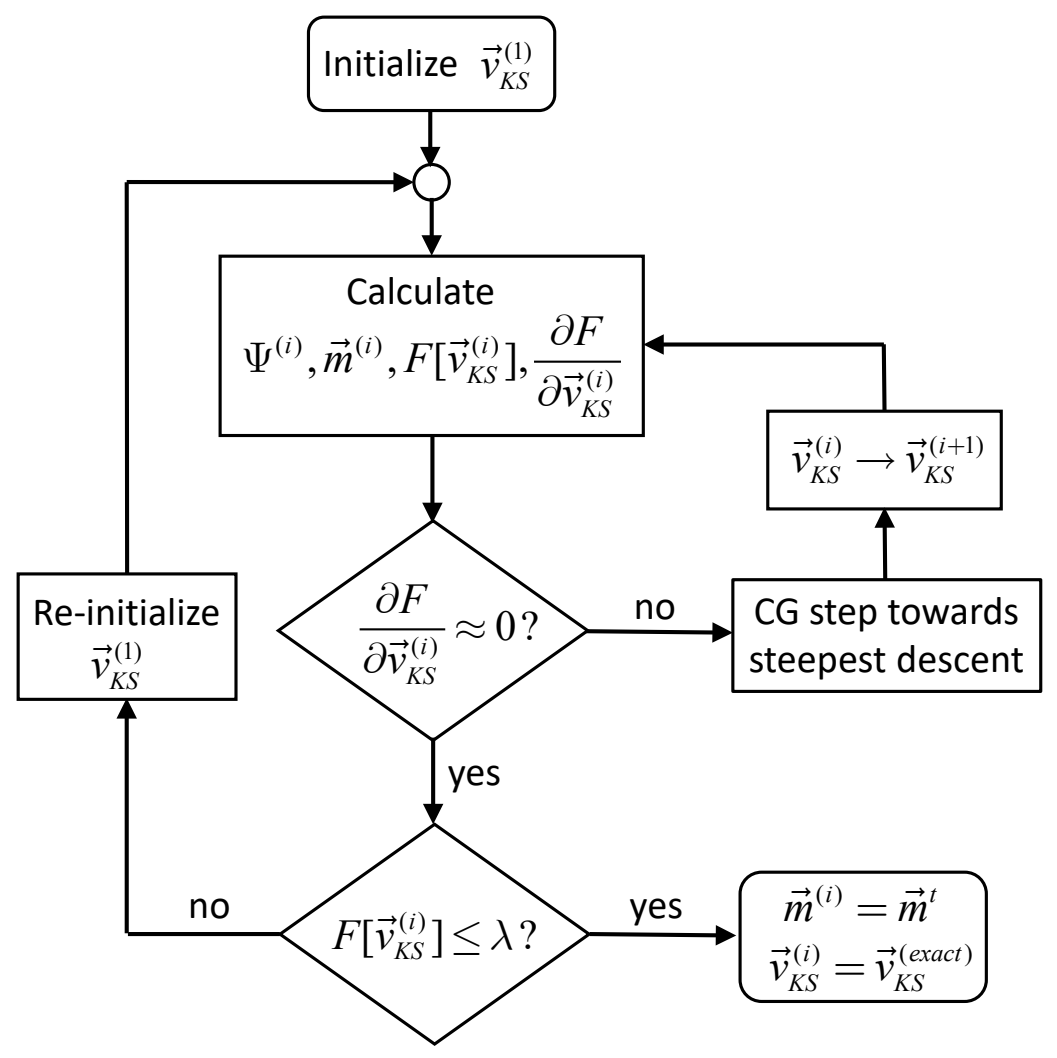

Figure 4.1: Flowchart of the CG routine used to calculate the exact KS 4-potential $\vec{v}_{\mathrm{KS}}$ giving rise to a target 4-density $\vec{m}^{t}$. 
in place. As stated, we begin from an exact target solution $\vec{m}_{k}^{t}$, and then solve the KS equation for a given initial choice of $\vec{v}_{\mathrm{KS}}$ (e.g., the bare external potential). The corresponding KS density and magnetizations are fed into the CG routine frprmn of Ref. [115], where the cost functional and gradients are calculated. After finding a step direction, the KS equation is recalculated at the new configuration space site, where the new density and magnetization can be used to continue towards convergence. The scheme is illustrated as a flowchart in Fig. 4.1.

Finding a global minimum in a multidimensional parameter space is in general a difficult task, and it often happens that a search ends up in a local minimum. We have found that $\lambda=F \leq 10^{-16}$ yields a 4-density to within $10^{-7}$ of $\vec{m}_{k}^{t}$, where $\lambda$ is our threshold for convergence. Occasionally, however, the CG routine will get stuck in a local minimum that is greater than $\lambda$. When this happens, we restart the CG iteration from a slightly different initial $\vec{v}_{\mathrm{KS}}$. At times, several restarts are needed before the global minimum is reached.

The KS inversion routine described above is for a fixed value of $U_{0}$. In practice, we embed the algorithm shown in Fig. 4.1 in a loop where $U_{0}$ is stepped over the desired range. The solution for $\vec{v}_{\mathrm{KS}}$ at a given value of $U_{0}$ is used to initialize the search at the next value of $U_{0}$.

Lastly, since the target 4-density $\vec{m}_{k}^{t}$ was obtained by exact diagonalization, the external 4-potential is known and the exact xc scalar potential and magnetic field (and hence the xc torque) are immediately obtained. 


\subsubsection{Nearest-neighbor interactions and the zero-torque the- orem}

Our choice to include n.n. interactions in $\hat{H}_{\text {lat }}$ is motivated by the fact that for the usual o.s. Hubbard model (where $U_{1}=0$ ) the exact exchange potential and exchange magnetic field are given by $[105,106,111]$

$$
V_{\mathrm{x}, k}^{\text {o.s. }}=-\frac{U_{0}}{2} n_{k}, \quad \mathbf{B}_{\mathrm{x}, k}^{\text {o.s. }}=-\frac{U_{0}}{2} \mathbf{m}_{k} .
$$

Evidently, $\mathbf{B}_{x, k}^{\text {o.s. }}$ is parallel to the magnetization; hence, for the o.s. Hubbard model, the xc torques are a pure correlation effect which, one can expect, makes them more difficult to approximate. For exchange effects to produce torques, more general interactions than o.s. are required.

Let us first consider the half-filled Hubbard dimer $(P=2)$ [105]: including n.n. interactions, one finds

$$
V_{\mathrm{x}, k}^{\text {dimer }}=-U_{1}-\frac{U_{0}-U_{1}}{2} n_{k}, \quad \mathbf{B}_{\mathrm{x}, k}^{\text {dimer }}=-\frac{U_{0}-U_{1}}{2} \mathbf{m}_{k},
$$

which, again, does not produce any torques. We therefore need more lattice points.

For the 1/3-filled Hubbard trimer [106] $(P=3)$ with periodic boundary conditions and n.n. interactions, i.e., an equilateral triangle, Eq. (4.13) holds on all three lattice points. If one instead considers a Hubbard trimer with fixed boundaries (i.e., a short chain), one finds that the solution (4.13) holds on the midpoint, but there is no simple explicit solution on the first and the third point. Thus, exchange torques can arise on points 1 and 3 , but not on point 2 .

The simplest case where exchange torques can occur on all lattice sites is the $1 / 4$ - 

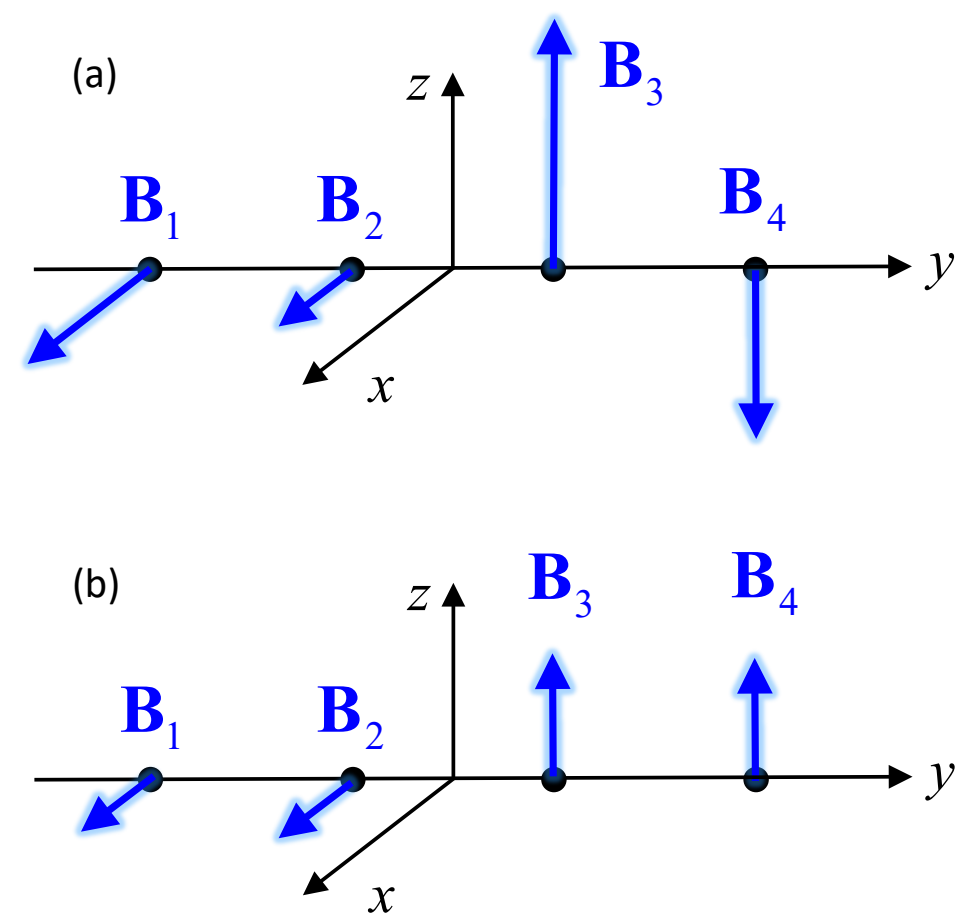

Figure 4.2: Hubbard tetramer with noncollinear coplanar magnetic field $\mathbf{B}$ (blue arrows). (a) Nonsymmetric field: $B_{x, 1}=0.2, B_{x, 2}=0.1, B_{z, 3}=0.3, B_{z, 4}=-0.2$. (b) $C_{2}$-symmetric field: $B_{x, 1}=B_{x, 2}=B_{z, 3}=B_{z, 4}=0.1$. In both cases, the scalar potential is $V_{1}=V_{4}=1, V_{2}=V_{3}=-1$. All local external and xc torques are along the $\pm y$-direction.

filled Hubbard tetramer $(P=4)$ with o.s. and n.n. interactions. In the following, we will consider tetramers in linear configuration, see Figure 4.2. Of course, correlation effects can produce torques in all the above systems, even without n.n. interactions.

A two-electron system without external magnetic field is always in a singlet ground state and hence nonmagnetic. To obtain a ground state with noncollinear magnetization, external magnetic fields are required (in the absence of spin-orbit coupling $[116,117])$.

In the following Sections, two cases with different symmetries will be considered. 
In both of them, the 4-point lattice is assumed to have equidistant sites, aligned along the $y$-direction (notice that the Hubbard lattice Hamiltonian is not necessarily tied to a specific real-space representation; however, assuming an explicit spatial geometry is conceptually helpful). The scalar potential will be taken to be nonuniform but symmetric with respect to the lattice center; we choose $V_{1}=V_{4}=1$ and $V_{2}=V_{3}=-1$ in both cases. This will allow us explore the different regimes within the interplay between confining scalar potential and electronic repulsion for the range of interaction strengths $0 \leq U_{0} \leq 10$.

We restrict the magnetic fields and the resulting magnetization to be coplanar, confined to the $x-z$ plane on each lattice point, but overall noncollinear (see Fig. 4.2). The resulting $\mathrm{xc}$ torques will lie along the $\pm y$-direction.

We obtain the ground-state energy, density, and magnetization on the lattice by exact diagonalization and via self-consistent Kohn-Sham. For the latter, we use three approximations [105]: exact exchange using the optimized effective potential approach (XX), exact exchange within the Slater approximation (XXS), and xc using the Singwi-Tosi-Land-Sjölander (STLS) scheme $[118,119]$. The latter is implemented within the scalar approximation, as detailed in Ref. [105].

$\mathrm{XX}$ is fully variational and hence satisfies the zero-torque theorem; XXS and STLS, on the other hand, are not variational and can violate the zero-torque theorem. Howver, as we will show below, the zero-torque theorem can be enforced by constrained optimization. The torque-corrected approximations defined in this way will be denoted as XXSc and STLSc, respectively.

Recall, the zero-torque theorem states that the xc magnetic field cannot exert a net torque on the system itself [94]. This in essence means that an electron cannot 
impose a torque on its own spin, as this would imply an electron can cause its own spin precession. While this is true for the exact xc magnetic field, a given approximation may violate this requirement, so that

$$
\int d \mathbf{r} \mathbf{m}(\mathbf{r}) \times \mathbf{B}_{\mathrm{xc}}^{\mathrm{app}}(\mathbf{r})=\mathbf{T}
$$

where $\mathbf{T}$ is a spurious macroscopic torque.

Kurzweil, Baer and Head-Gordon [120,121] showed how to enforce exact conditions on approximate local xc potentials by using constrained minimization with Lagrange multipliers. Here, we use a similar approach (but without Lagrange multipliers) to enforce the zero-torque theorem. The idea is to construct a counteracting field $\mathbf{b}(\mathbf{r})$ such that

$$
\int d \mathbf{r} \mathbf{m}(\mathbf{r}) \times \mathbf{b}(\mathbf{r})=\mathbf{T}
$$

and

$$
\|\mathbf{b}(\mathbf{r})\|^{2} \equiv \int d \mathbf{r}|\mathbf{b}(\mathbf{r})|^{2}=\min
$$

In other words, we are looking for a vector field which generates the same macroscopic torque, but whose integrated vector norm is as small as possible. Once such a $\mathbf{b}(\mathbf{r})$ has been found, we define

$$
\tilde{\mathbf{B}}_{\mathrm{xc}}^{\mathrm{app}}(\mathbf{r})=\mathbf{B}_{\mathrm{xc}}^{\mathrm{app}}(\mathbf{r})-\mathbf{b}(\mathbf{r})
$$

as a new approximation, which now satisfies the zero-torque theorem while remaining as close as possible to the original $\mathbf{B}_{\mathrm{xc}}^{\mathrm{app}}(\mathbf{r})$. We now show how to explicitly find $\mathbf{b}(\mathbf{r})$ on a lattice, distinguishing two cases. 


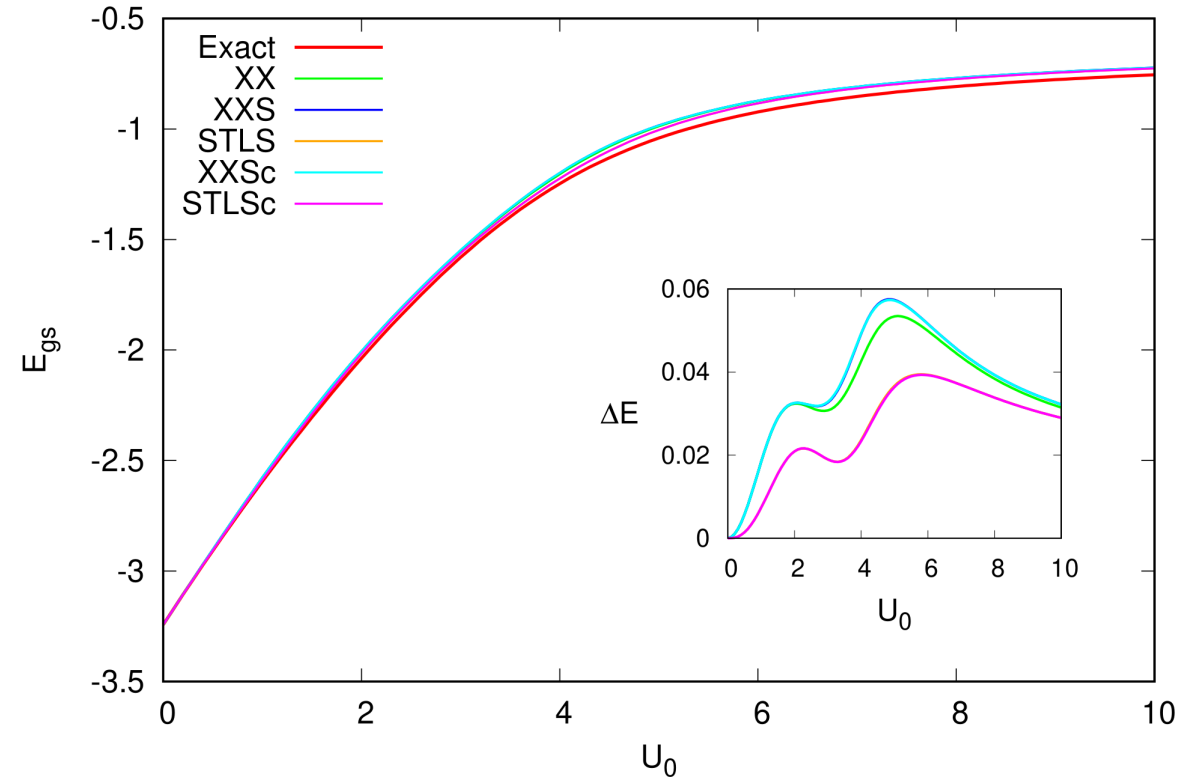

Figure 4.3: Ground-state energy of the Hubbard tetramer with nonsymmetric magnetic fields, see Fig. 4.2a, comparing the exact result with various SDFT approximations (see text). The difference between the exact solution and the SCDF approximations are shown in the inset. The lines for XXS and XXSc, as well as STLS and STLSc, respectively, are too close to be distinguished. 


\subsection{Nonsymmetric lattice}

We begin our discussion by considering a Hubbard tetramer where no distinct spatial or spin symmetries are present. In this nonsymmetric case (see Fig. 4.2a), we set $B_{x, 1}=0.2, B_{x, 2}=0.1, B_{z, 3}=0.3$, and $B_{z, 4}=-0.2$, with all other components zero. The external magnetic field defined in this way is coplanar in the $x-z$ plane, and hence all torques (external as well as xc) are along the (positive or negative) $y$-direction.

\subsubsection{Ground state energy}

In Fig. 4.3 we compare the exact ground-state energy of the nonsymmetric lattice with Kohn-Sham results using XX, XXS, STLS, XXSc and STLSc. The exact energy starts out at $E_{\mathrm{gs}}=-3.242$ for the noninteracting case $\left(U_{0}=0\right)$, reaches the value $E_{\mathrm{gs}}=-0.754$ for $U_{0}=10$, and approaches a limiting value of $E_{\mathrm{gs}}=-0.6107$ for $U_{0} \rightarrow \infty$. The crossover between the weakly and strongly correlated regimes occurs around $U_{0} \sim 4$. The agreement between the exact energy and the Kohn-Sham energies is very good for all values of $U_{0}$. The inset shows that the largest errors of $E_{\mathrm{gs}}$ occur around $U_{0}=5$, reaching around $5 \%$ for XX and XXS and slightly less for STLS.

The xc torque correction of XXS and STLS has a negligible impact on the total energy; in Fig. 4.3, the lines for XXS and XXSc are essentially on top of each other, as are the lines for STLS and STLSc. The reason for this is easy to see: to lowest order perturbation theory, the energy shift caused by a magnetic field $\mathbf{B}^{\prime}(\mathbf{r})$ is

$$
\Delta E=\int d \mathbf{r} \mathbf{m}(\mathbf{r}) \cdot \mathbf{B}^{\prime}(\mathbf{r}),
$$


Table 4.1: Exact density and magnetization data for the nonsymmetric and $C_{2^{-}}$ symmetric lattices, in the noninteracting $\left(U_{0}=0\right)$ and strongly correlated $\left(U_{0}=10\right)$ limit.

\begin{tabular}{c|cccc|cccc}
\hline \hline & \multicolumn{4}{|c}{ nonsymmetric } & \multicolumn{5}{c}{$C_{2}$-Symmetric } \\
$U_{0}$ & $n_{1}$ & $n_{2}$ & $n_{3}$ & $n_{4}$ & $n_{1}$ & $n_{2}$ & $n_{3}$ & $n_{4}$ \\
\hline 0 & 0.037 & 0.961 & 0.967 & 0.034 & 0.036 & 0.964 & 0.964 & 0.036 \\
10 & 0.911 & 0.095 & 0.891 & 0.102 & 0.518 & 0.482 & 0.482 & 0.518 \\
& $m_{1}$ & $m_{2}$ & $m_{3}$ & $m_{4}$ & $m_{1}$ & $m_{2}$ & $m_{3}$ & $m_{4}$ \\
\hline 0 & 0.017 & 0.291 & 0.304 & 0.005 & 0.007 & 0.143 & 0.143 & 0.007 \\
10 & 0.911 & 0.088 & 0.890 & 0.102 & 0.518 & 0.477 & 0.477 & 0.518 \\
& & & & & & & & \\
& $m_{x, 1}$ & $m_{x, 2}$ & $m_{x, 3}$ & $m_{x, 4}$ & $m_{x, 1}$ & $m_{x, 2}$ & $m_{x, 3}$ & $m_{x, 4}$ \\
\hline 0 & -0.010 & -0.131 & 0.099 & 0.005 & -0.005 & -0.103 & 0.098 & 0.005 \\
10 & -0.911 & -0.088 & 0.005 & 0.004 & -0.518 & -0.477 & 0.004 & 0.009 \\
& & & & & & & & \\
& $m_{z, 1}$ & $m_{z, 2}$ & $m_{z, 3}$ & $m_{z, 4}$ & $m_{z, 1}$ & $m_{z, 2}$ & $m_{z, 3}$ & $m_{z, 4}$ \\
\hline 0 & 0.013 & 0.260 & -0.288 & -0.002 & 0.005 & 0.098 & -0.103 & -0.005 \\
10 & 0.007 & -0.006 & -0.890 & -0.102 & 0.009 & 0.004 & -0.477 & -0.518 \\
& $\left\langle\hat{S}_{x}\right\rangle$ & $\left\langle\hat{S}_{y}\right\rangle$ & $\left\langle\hat{S}_{z}\right\rangle$ & $\left\langle\hat{\mathbf{S}}^{2}\right\rangle$ & $\left\langle\hat{S}_{x}\right\rangle$ & $\left\langle\hat{S}_{y}\right\rangle$ & $\left\langle\hat{S}_{z}\right\rangle$ & $\left\langle\hat{\mathbf{S}}^{2}\right\rangle$ \\
\hline 0 & -0.018 & 0 & -0.008 & 0.100 & -0.002 & 0 & -0.002 & 0.023 \\
10 & -0.495 & 0 & -0.496 & 1.488 & -0.491 & 0 & -0.491 & 1.477 \\
\hline \hline
\end{tabular}

where $\mathbf{m}(\mathbf{r})$ is the magnetization of the unperturbed ground state. Thus, a transverse magnetic field [which is perpendicular to $\mathbf{m}(\mathbf{r})$ for all $\mathbf{r}$ ] only contributes to the total energy to second and higher order. The xc torque correction has the form of a transverse magnetic field added to the xc magnetic field to be corrected.

\subsubsection{Density and magnetization}

Figure 4.4 shows the density $n_{k}$ (top panel) and the $x$ - and $z$-components of the magnetization, $m_{x, k}$ and $m_{z, k}$ (middle and bottom panels). At $U_{0}=0$, the density 

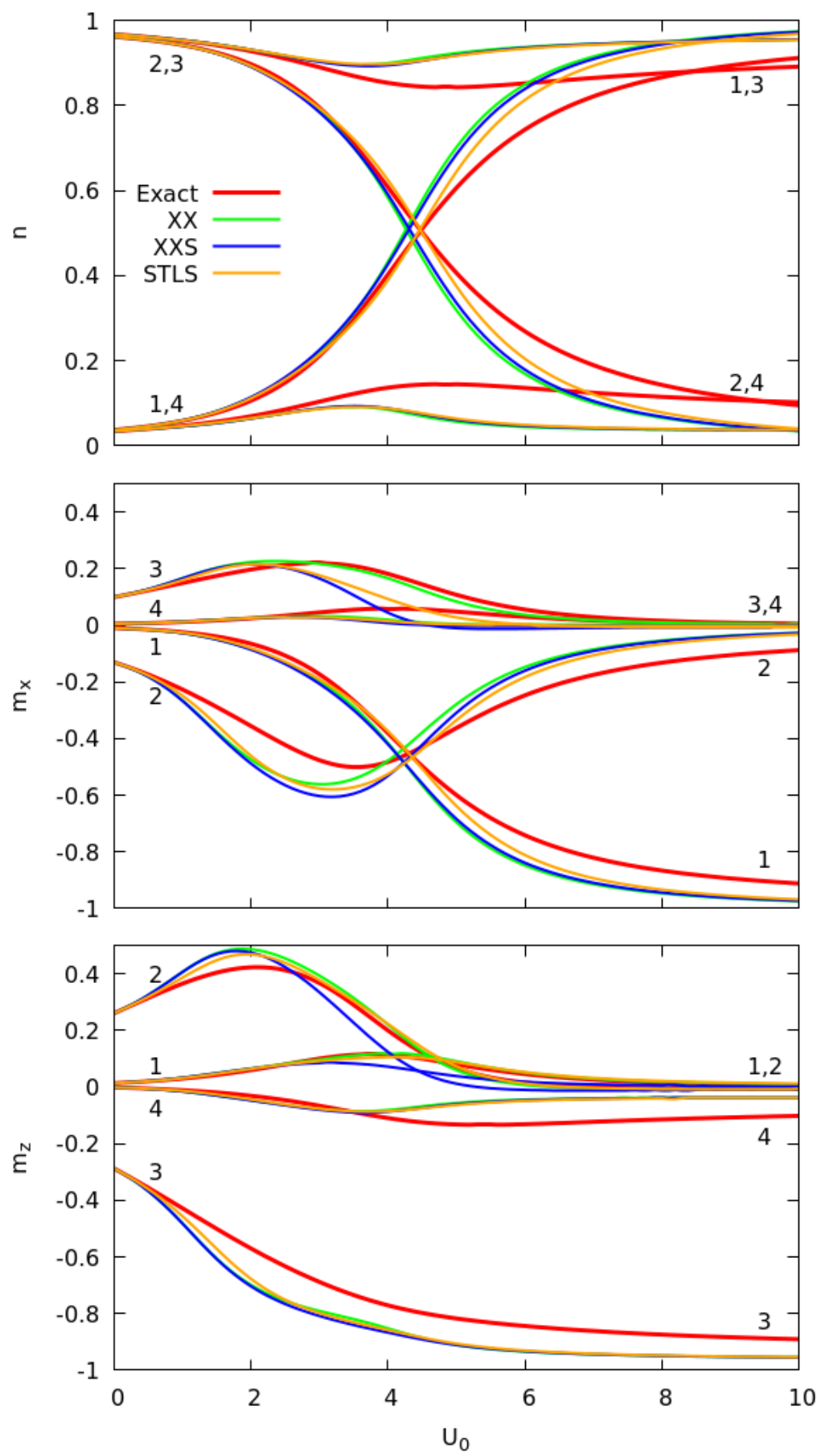

Figure 4.4: Density (top) and $x$ - and $z$-components (middle and bottom) of the magnetization of the nonsymmetric Hubbard tetramer. The numbers indicate lattice sites. 
is almost entirely concentrated on points 2 and 3 . On these two points, the scalar potential is lower than on sites 1 and 4 , forming a very simple potential well. As $U_{0}$ increases, the electronic repulsion leads to a redistribution of the site occupation, so the electrons can better avoid each other (keep in mind that our model includes the n.n. interaction $U_{1}$ ); the crossover clearly occurs around $U_{0} \sim 4$. At $U_{0}=10$, lattice sites 1 and 3 are almost fully occupied, whereas sites 2 and 4 are almost empty, see Table 4.1 for details. This density distribution minimizes the Coulomb repulsion due to $U_{0}$ and $U_{1}$, while still taking advantage of the potential energy minimum in the middle of the lattice; sites 1 and 3 are favored over sites 2 and 4 because the magnetic field is largest on site 3, which gives the largest contribution of negative Zeeman energy. This behavior is somewhat exaggerated, but overall well reproduced, by the approximate Kohn-Sham calculations. Again, XXSc and STLSc give results very similar to XXS and STLS, and are not shown here.

The magnetization reveals further details which illustrate the behavior of the system as the interaction strength increases. At $U_{0}=0$, the system is only relatively weakly magnetized; on the other hand, at $U_{0}=10$, the magnitude of the magnetization vector on each lattice site is practically identical with the density. Furthermore, Table 4.1 tells us that at $U_{0}=10$ the magnetization vector is (anti)aligned with the applied magnetic field on each lattice site: on sites 1 and 2, the magnetization points almost completely in the negative $x$-direction, and on sites 3 and 4 it points in the negative $z$-direction. Referring back to Fig. 4.2a, we see that the magnetization vector is opposite to the applied magnetic field on sites 1,2 , and 3 , and parallel to it on site 4 . The physical meaning of this is that in the strongly correlated limit, the electrons not only localize spatially, they also align their spin quantization axis with 
the local magnetic field.

Magnetic systems can be characterized by the expectation values of the total spin operator $\hat{\mathbf{S}}$ and its squared magnitude, $\hat{\mathbf{S}}^{2}$. In collinear magnetic systems, the twoelectron wave functions are pure singlets or triplets, and one finds $\left\langle\hat{S}_{z}\right\rangle=M_{s}$ with $M_{s}=0, \pm 1$ and $\left\langle\hat{\mathbf{S}}^{2}\right\rangle=S(S+1)$ with $S=0,1$. In the noncollinear case, the twoelectron wave functions are of mixed spin character, and the expectation values $\langle\hat{\mathbf{S}}\rangle$ and $\left\langle\hat{\mathbf{S}}^{2}\right\rangle$ will not be integers. The explicit numbers for our systems are given in Table 4.1. At $U_{0}=0$, the exact two-electron wave function has $95 \%$ singlet character [122], and $\left\langle\hat{S}_{x}\right\rangle,\left\langle\hat{S}_{z}\right\rangle$ and $\left\langle\hat{\mathbf{S}}^{2}\right\rangle$ are very small but not zero. In the limit $U_{0} \rightarrow \infty$, the singlet-triplet ratio is 1:3, and one finds $\left\langle\hat{S}_{x}\right\rangle=\left\langle\hat{S}_{z}\right\rangle=-1 / 2$ and $\left\langle\hat{\mathbf{S}}^{2}\right\rangle=3 / 2$. At $U_{0}=10$, where the exact wave function has $74 \%$ triplet character, we are close to this limit.

\subsubsection{Hartree and xc potentials and xc magnetic fields}

Figure 4.5 compares the exact Hartree potential, Eq. (4.6), and the exact xc potential, respectively, with the corresponding XX, XXS(c) and STLS(c) approximations. The $x$ - and $z$-components of the xc magnetic fields are shown in Fig. 4.6. There is some numerical noise in the exact xc potentials and magnetic fields as $U_{0}$ approaches 10 , since our inversion procedure of the Kohn-Sham equation becomes more difficult to converge in the strongly correlated regime.

$V_{\mathrm{H}, k}, V_{\mathrm{xc}, k}$, and $\mathbf{B}_{\mathrm{xc}, k}$ are responsible for reproducing the exact density and magnetization in the noninteracting Kohn-Sham system. As the system passes from the weakly to the strongly interacting regime with increasing $U_{0}$, density gets shifted from site 2 to site 1, and sites 1 and 3 become almost fully magnetized. The Kohn-Sham 

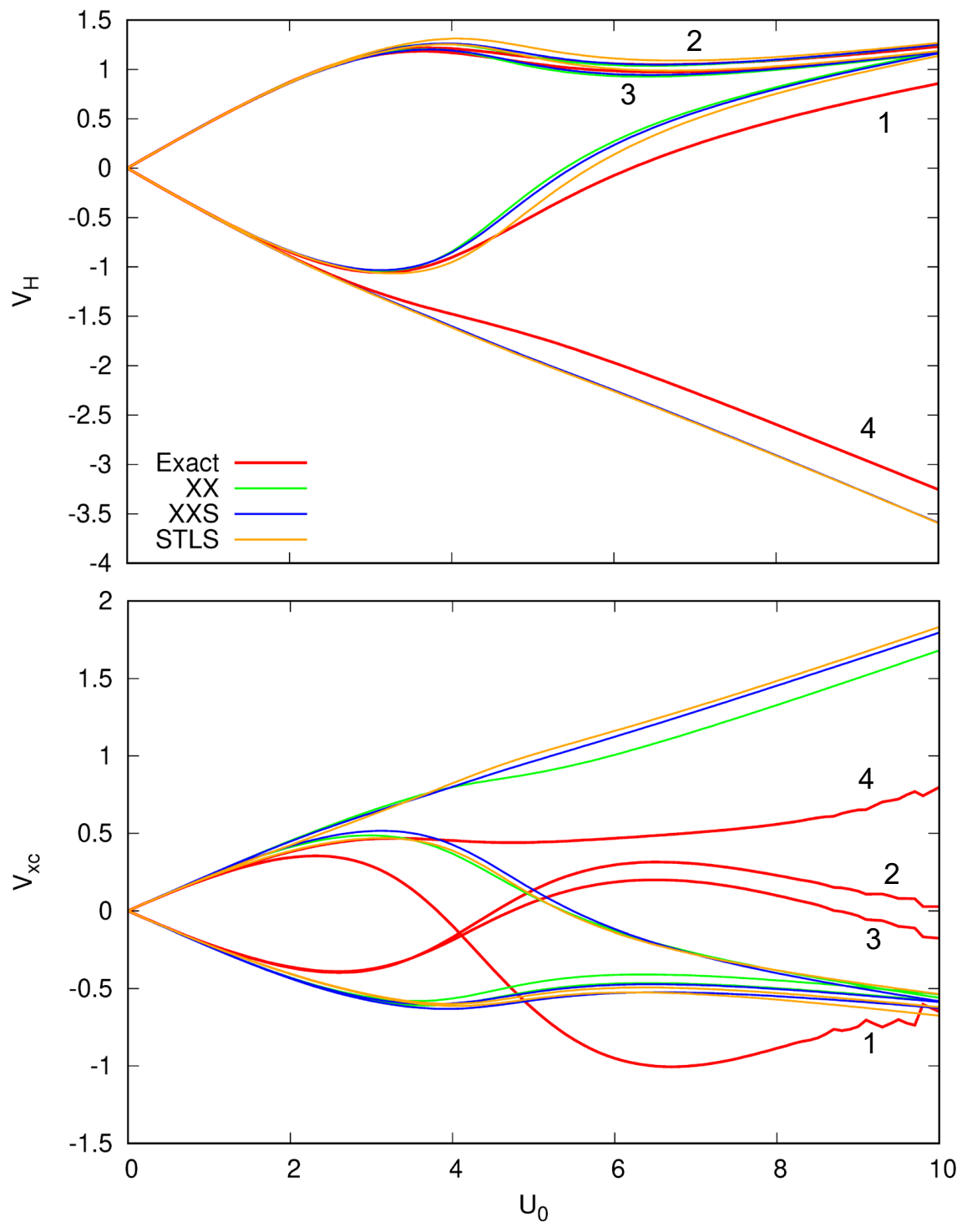

Figure 4.5: Hartree (top) and xc potential (bottom) of the nonsymmetric Hubbard tetramer. The numbers indicate lattice sites. 

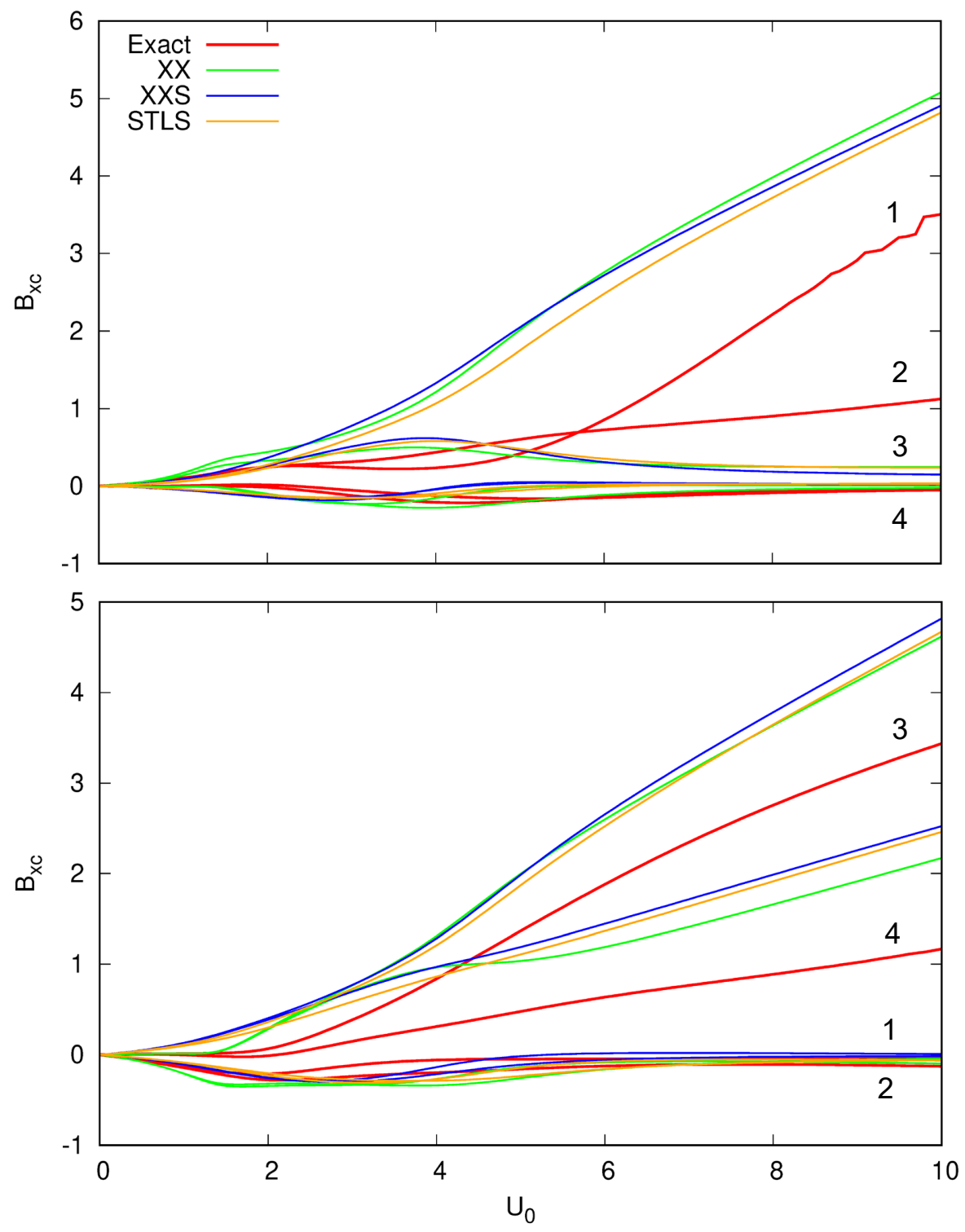

Figure 4.6: $x$ - (top) and $z$-component (bottom) of the xc magnetic field of the nonsymmetric Hubbard tetramer. The numbers indicate lattice sites. 
system accomplishes this through an intricate interplay between Hartree and xc effects. For instance, comparing $V_{\mathrm{H}, k}$ and $V_{\mathrm{xc}, k}$, we see that the xc potential partially counteracts the Hartree potential, especially on sites 1 and 4 .

The xc magnetic field displays a striking enhancement over the external magnetic field, by about an order of magnitude, in the strongly correlated regime. These large field strengths are necessary so that the Kohn-Sham system can create the alignment of the local magnetic moments with the external field in the strongly correlated limit. Looking again at Table 4.1, we see that the magnetic moments are almost completely aligned along the $x$-direction on sites 1 and 2 , and along the $z$-directions on sites 3 and 4. Correspondingly, the $x$ - and $z$-components of $\mathbf{B}_{\mathrm{xc}, k}$ are very strongly enhanced on these sites.

The approximate xc potentials and magnetic fields agree well with the exact results until about $U_{0}=3$. For larger interaction strengths, considerable differences appear. For example, the xc potentials on sites 1, 2, and 3 change sign, which is not reproduced by any of the approximations, and the xc potential on site 4 has the wrong limiting behavior for large $U_{0}$. Similar discrepancies are observed for the xc magnetic fields. This helps explain the differences between exact and approximate densities and magnetizations in Fig. 4.4.

\subsubsection{Exact and approximate xc torques}

Let us now examine the xc torques of the nonsymmetric tetramer. Figure 4.7 shows a comparison of the exact $\boldsymbol{\tau}_{\mathrm{xc}, k}$ (full lines) with the torque caused by the externally applied magnetic field, $\boldsymbol{\tau}_{\text {ext }, k}$ (dashed lines). The latter is defined in analogy with Eq. (4.3), with $\mathbf{B}_{\mathrm{xc}, k}$ replaced by $\mathbf{B}_{k}$. Both torques are along the $y$-direction on all lattice 


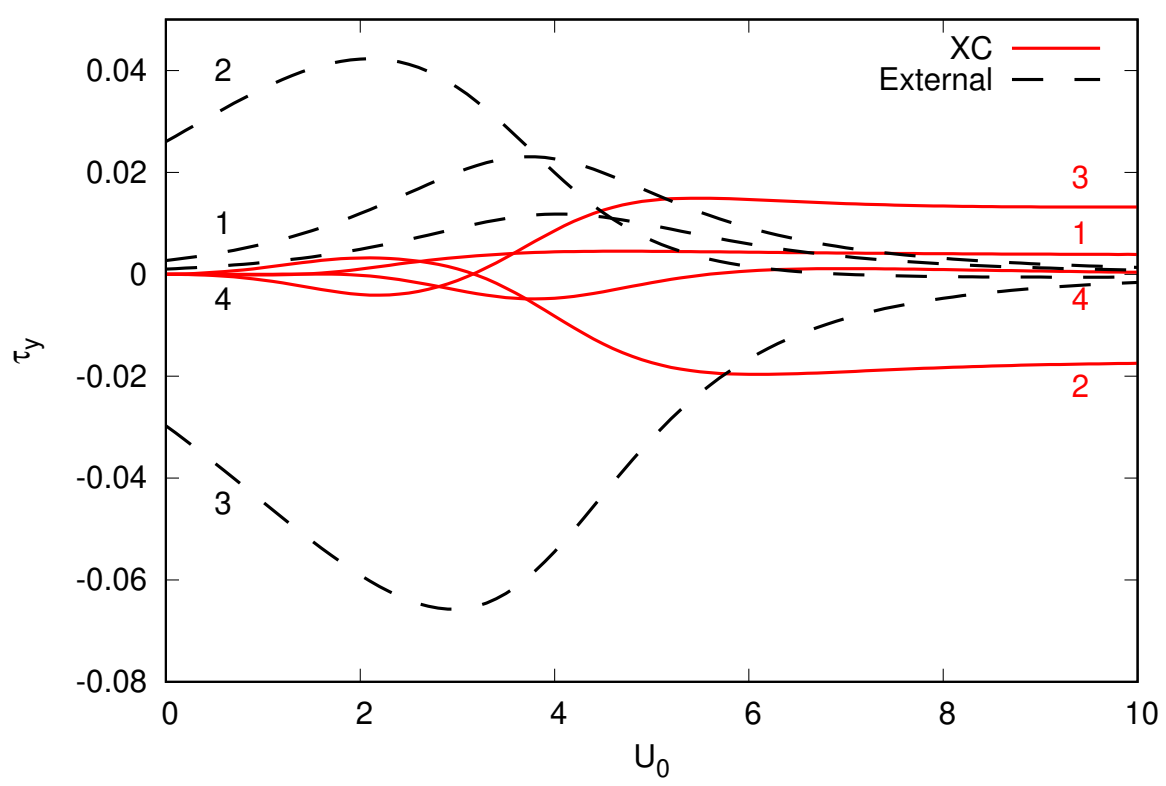

Figure 4.7: Comparison of xc torque $\boldsymbol{\tau}_{\mathrm{xc}, k}$ (red, full lines) and external torque $\boldsymbol{\tau}_{\text {ext }, k}$ (black, dashed lines) of the nonsymmetric tetramer. All torques only have a $y$ component. 

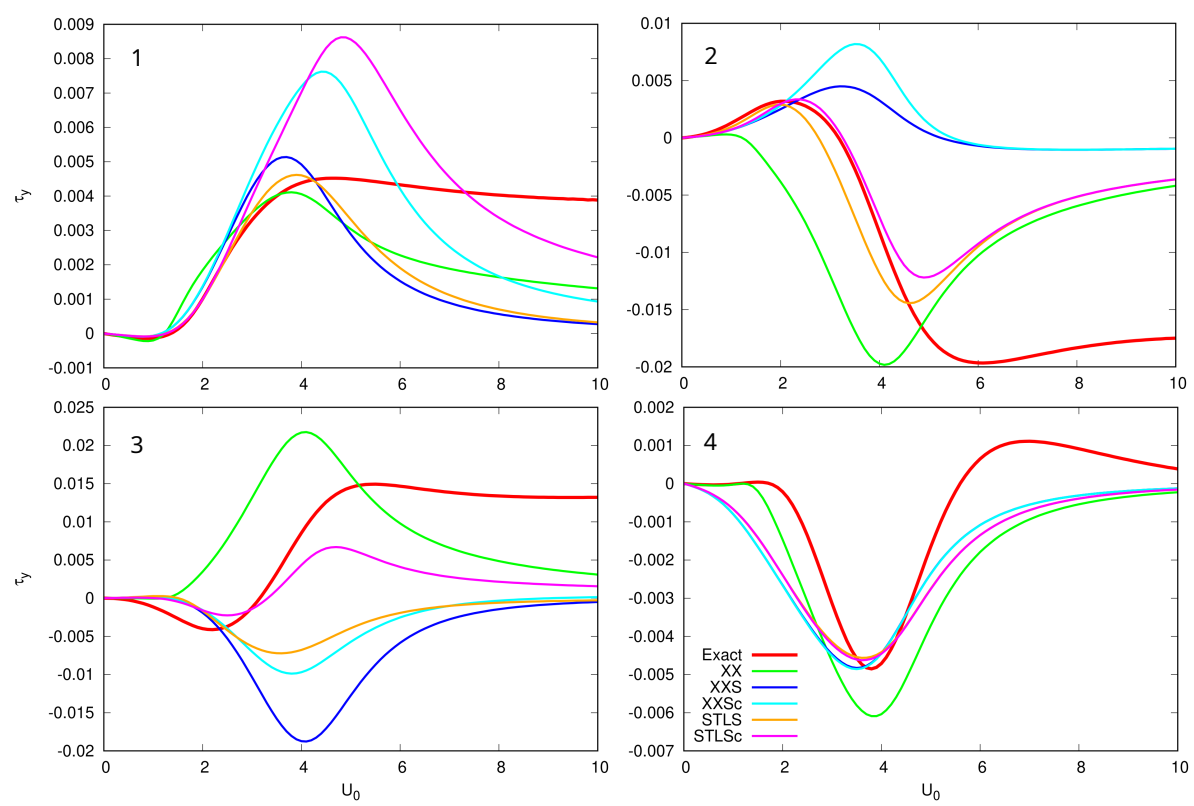

Figure 4.8: Comparison of exact xc torque $\boldsymbol{\tau}_{\mathrm{xc}, k}$ and xc torque approximations with and without torque correction for the nonsymmetric tetramer, on the four lattice sites. All torques only have a $y$-component.

sites. It can be seen that the xc torques on each lattice site add up to zero, as do the external torques (as they must, since the system is in equilibrium).

For weak to moderate interaction strengths, the external torque dominates over the xc torque, with maxima around $U_{0}=2$ to 4 , depending on the lattice site. This indicates that in this regime the external magnetic field has a sizable component perpendicular to the magnetization. As the system localizes for $U_{0} \gtrsim 4$, the magnetization on each lattice site aligns with the external magnetic field, as discussed in the previous subsection, so that $\tau_{\text {ext }, k}$ goes to zero.

The xc torques, on the other hand, are small for weak interactions, but become larger than the external torque on sites 2 and 3 after the crossover into the strongly correlated regime. This means that, on these sites, the xc magnetic field does not 
quite align with the local magnetization. Looking at Table 4.1, we see that at $U_{0}=10$ sites 2 and 3 are almost, but not fully magnetized and aligned with the local external fields; furthermore, as Fig. 4.6 shows, the xc magnetic fields are very large on sites 2 and 3. Together, this results in a finite $\boldsymbol{\tau}_{\mathrm{xc}, k}$, which is necessary for the Kohn-Sham system to reproduce all subtle details of the magnetization of the interacting system.

The xc torques obtained with XX, XXS, STLS, XXSc and STLSc are presented in Fig. 4.8 for each lattice point. The xc torques are much smaller on sites 1 and 4 than on sites 2 and 3, which means that the performance of the approximations is more crucial on the latter sites. Compared to the exact xc torques, the approximate xc torques generally have the right order of magnitude, which is reassuring; however, there are some important details, depending on the lattice site and the level of approximation.

On site 1, all approximations perform well for small to moderate interactions, but fail to reproduce the finite xc torque for large interaction strengths; XXSc and STLSc overshoot around $U_{0}=5$. On site 4 , the quantitative agreement is quite good throughout, for all approximations.

On sites 2 and 3 , the exact xc torque changes sign between $U_{0}=3$ and 4 . This trend is not reproduced by XX, XXS and XXSc. STLS performs much better for small to moderate interaction strengths, including the sign change, with STLSc leading to slight improvements over STLS. However, all approximations produce a vanishing xc torque in the strongly correlated regime, instead of approaching a finite limit.

How important are the xc torques? We have already seen that they give only a very small contribution to the total energy. We have carried out self-consistent calculations with XX, XXS and STLS where we only include the longitudinal components of $\mathbf{B}_{\mathrm{xc}, k}$. 
We find that the magnetization on the individual sites does not change much for the nonsymmetric case.

\subsection{Lattice with $C_{2}$ symmetry}

We now consider a magnetic field distribution which has $C_{2}$ symmetry, as illustrated in Fig. 4.2b. The symmetry is with respect to rotation about an axis which lies at an angle of $45^{\circ}$ between the $x$ and $z$ axes. Specifically, we choose $B_{x, 1}=B_{x, 2}=B_{z, 3}=$ $B_{z, 4}=0.1$.

Let us consider a $P$-point lattice, where $P$ is an even number (the case where $P$ is odd will lead to similar findings, as will be briefly discussed at the end of this Section). We assume, for now, that the system is noninteracting (i.e., $U_{0}=U_{1}=0$ ), and we write the single-particle wave functions as vectors of length $2 P$ :

$$
\boldsymbol{\Psi}=\left(\begin{array}{c}
\psi_{1 \uparrow} \\
\psi_{1 \downarrow} \\
\psi_{2 \uparrow} \\
\psi_{2 \downarrow} \\
\vdots \\
\psi_{P \uparrow} \\
\psi_{P \downarrow}
\end{array}\right)
$$

where $\psi_{k \uparrow, \downarrow}$ are the spin-up and -down components of $\boldsymbol{\Psi}$ on the $k$ th lattice point. The 
$x, y, z$-components of the magnetization vector on the $k$ th lattice site are

$$
m_{i, k}=\left(\psi_{k \uparrow}^{*}, \psi_{k \downarrow}^{*}\right) \sigma_{i}\left(\begin{array}{c}
\psi_{k \uparrow} \\
\psi_{k \downarrow}
\end{array}\right), \quad k=1, \ldots, P
$$

where $\sigma_{i}$ are the Pauli matrices, with $i=x, y, z$.

The (noninteracting) Hamiltonian which determines $\boldsymbol{\Psi}$ is a $2 P \times 2 P$ matrix of the form

$$
\begin{aligned}
\underline{\underline{H}} & =\underline{\underline{T}}+\underline{\underline{V}}+\underline{\underline{B}} \\
& =-\left[\operatorname{diag}_{+1}(t)+\operatorname{diag}_{-1}(t)\right] \otimes \sigma_{0} \\
& +\operatorname{diag}\left(V_{1}, \ldots, V_{P}\right) \otimes \sigma_{0} \\
& +\sum_{i=x, y, z} \operatorname{diag}\left(B_{i 1}, \ldots, B_{i P}\right) \otimes \sigma_{i} .
\end{aligned}
$$

Here, $\operatorname{diag}\left(a_{1}, \ldots, a_{P}\right)$ denotes a $P \times P$ matrix with the diagonal elements $a_{1}, \ldots, a_{P}$ and all other elements zero; $\operatorname{diag}_{ \pm 1}(t)$ denotes a matrix where the first upper/lower off-diagonal elements all have the value $t . \sigma_{0}$ is the $2 \times 2$ unit matrix, and $\otimes$ denotes the Kronecker product.

The Hamiltonian matrix (4.21) has a block-tridiagonal form, where each diagonal block is $2 \times 2$ in size and contains the scalar potentials and magnetic fields on the respective lattice sites. The off-diagonal blocks are $2 \times 2$ unit matrices multiplied by the hopping parameter $-t$.

Now consider a unitary transformation described by a matrix $\underline{\underline{M}}$, which transforms 
the Schrödinger equation into

$$
\underline{\underline{M}} \underline{\underline{H}} \underline{\underline{M}}^{-1} \underline{\underline{M}} \Psi=E \underline{\underline{M}} \Psi
$$

We want to determine how this transformation affects the magnetization (4.20) for cases of special symmetry. Specifically, we consider scalar potentials that are symmetric about the lattice midpoint, i.e.,

$$
V_{k}=V_{P-k+1}
$$

In the following, we limit ourselves to coplanar magnetic fields; to be specific, we consider magnetic fields in the $x-z$ plane. Assuming that the lattice is aligned along the $y$-direction, we impose $C_{2}$ symmetry with respect to rotation about the $x$-axis; an example is given in Fig. 4.9 .

$C_{2}$ symmetric magnetic fields are characterized by the two conditions

$$
\begin{aligned}
& B_{x, k}=B_{x, P-k+1}, \\
& B_{z, k}=-B_{z, P-k+1} .
\end{aligned}
$$

This suggests the following transformation matrix:

$$
\underline{\underline{M}}=\left(\begin{array}{ccc}
\underline{0} & \ldots & \sigma_{x} \\
\vdots & \ddots & \vdots \\
\sigma_{x} & \cdots & \underline{0}
\end{array}\right),
$$

which satisfies $\underline{\underline{M}}=\underline{\underline{M}}^{\dagger}=\underline{\underline{M}}^{-1}$. We need to show that this operator leaves the 

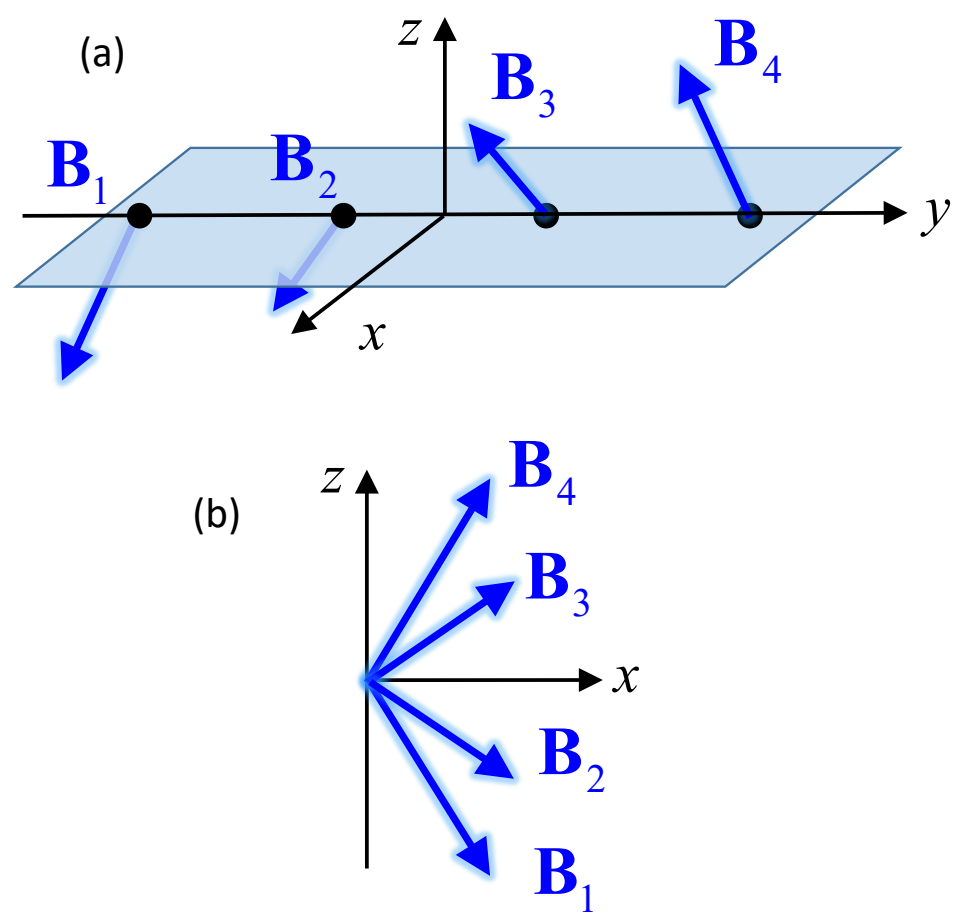

Figure 4.9: (a) Example of a magnetic field with $C_{2}$ symmetry on a 4-point lattice. (b) View along the $y$-axis. 
Hamiltonian (4.21) invariant, i.e.,

$$
\underline{\underline{M}} \underline{\underline{H}} \underline{\underline{M}}^{-1}=\underline{\underline{H}} .
$$

The invariance of $\underline{\underline{V}}$ and $\underline{\underline{T}}$ follows immediately from

$$
\sigma_{x} \sigma_{0} \sigma_{x}=\sigma_{0}
$$

For the invariance of $\underline{\underline{B}}$, the transformation must comply with the $C_{2}$ symmetry of the magnetic field. This follows immediately from

$$
\begin{aligned}
\sigma_{x} \sigma_{x} \sigma_{x} & =\sigma_{x} \\
\sigma_{x} \sigma_{z} \sigma_{x} & =-\sigma_{z} .
\end{aligned}
$$

To calculate the magnetization, we insert the transformed eigenfunction, $\underline{\underline{M}} \boldsymbol{\Psi}$, into Eq. (4.20), where

$$
\underline{\underline{M}} \boldsymbol{\Psi}=\left(\begin{array}{c}
\psi_{P \downarrow} \\
\psi_{P \uparrow} \\
\vdots \\
\psi_{1 \downarrow} \\
\psi_{1 \uparrow}
\end{array}\right)
$$

This gives the final result

$$
\begin{aligned}
& m_{x, k}=m_{x, P-k+1} \\
& m_{z, k}=-m_{z, P-k+1} .
\end{aligned}
$$


In other words, the magnetization also has $C_{2}$ symmetry, as expected.

We conclude this Section with three remarks.

- If the number of lattice sites $P$ is odd, $C_{2}$ symmetry requires that in the central site the magnetic field and the magnetization point along the $x$-direction. The definition of $\underline{\underline{M}}$ must be recast by putting $\sigma_{0}$ as the central block of $\underline{\underline{M}}$. The proof then goes through as before.

- The symmetry property of the magnetization, Eqs. (4.32) and (4.33), remains intact when interactions are present. This is because the Hamiltonian of the interacting system remains invariant under $C_{2}$ symmetry transformations. This implies that the Kohn-Sham system also has a magnetization which satisfies conditions (4.32) and (4.33), which is intuitively clear since the exchange-correlation magnetic field is evaluated self-consistently with a magnetization of the $C_{2}$ symmetry type. Our numerical results confirm this.

- Here, we considered $C_{2}$ symmetry with respect to rotations about the $x$-axis, which made the proof simpler. The lattice Hamiltonian has $C_{2}$ symmetry with respect to rotations about an axis that lies at a $45^{\circ}$ angle between the $x$ and the $z$ axis. Equations (4.32) and (4.33) then turn into the single condition

$$
m_{x, k}=m_{z, P-k+1}
$$




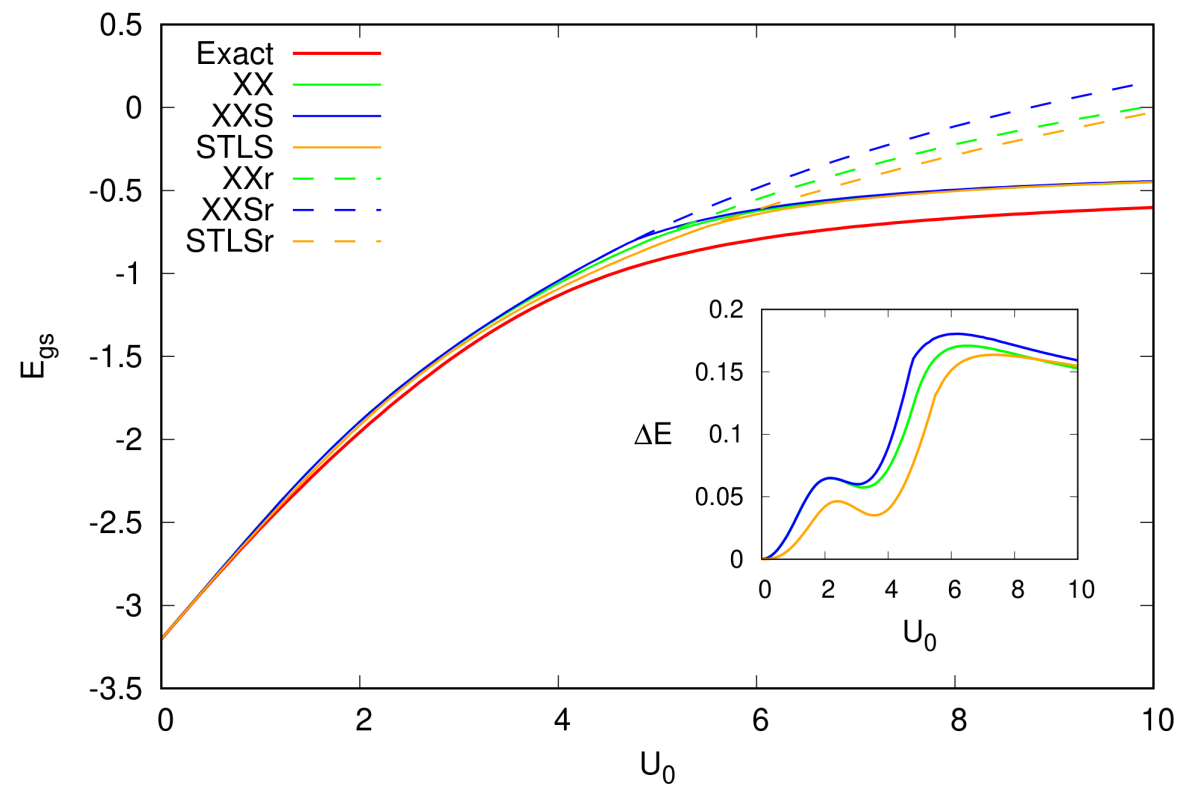

Figure 4.10: Ground-state energy of the Hubbard tetramer with $C_{2}$ symmetry. Dashed lines are symmetry enforced calculations, while the solid lines were allowed to break the symmetry of the system. The difference between the exact solution and the corresponding (unrestricted) approximations are plotted in the inset. 

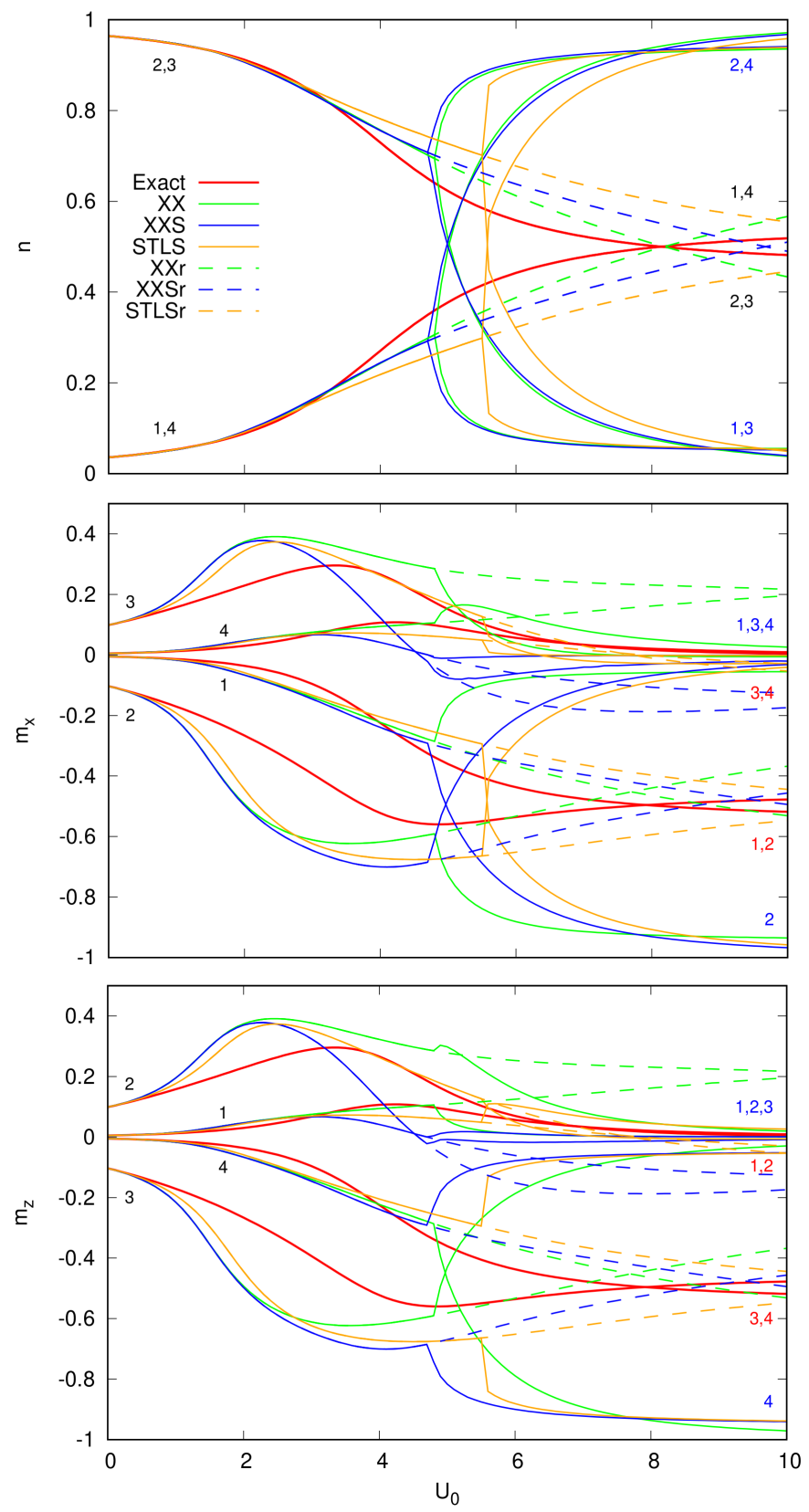

Figure 4.11: Density (top) and $x$ - and $z$-components (middle and bottom) of the magnetization of the Hubbard tetramer with $C_{2}$ symmetry. The numbers indicate lattice sites. 

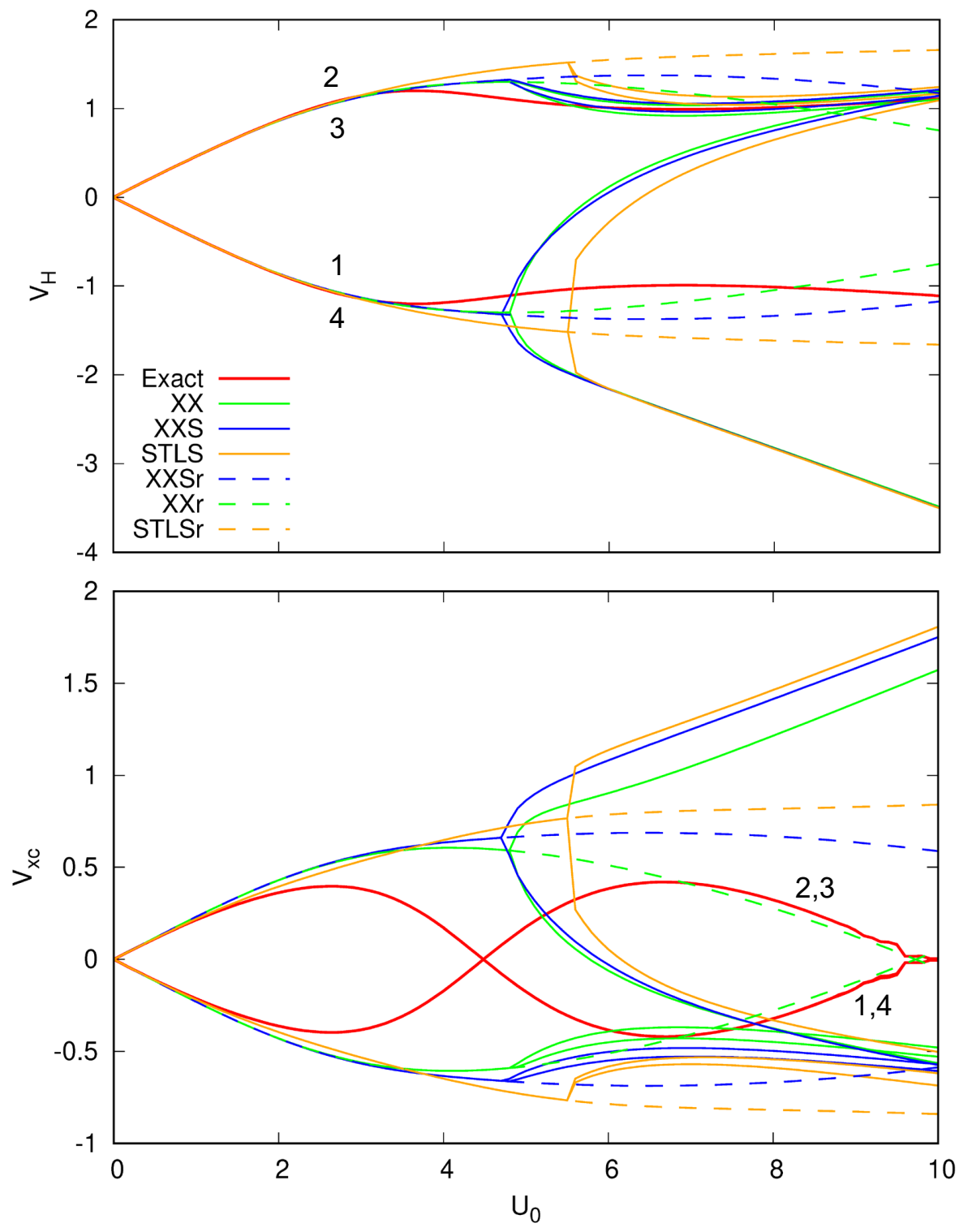

Figure 4.12: Hartree (top) and xc potential (bottom) of the $C_{2}$-symmetric Hubbard tetramer. The numbers indicate lattice sites. 

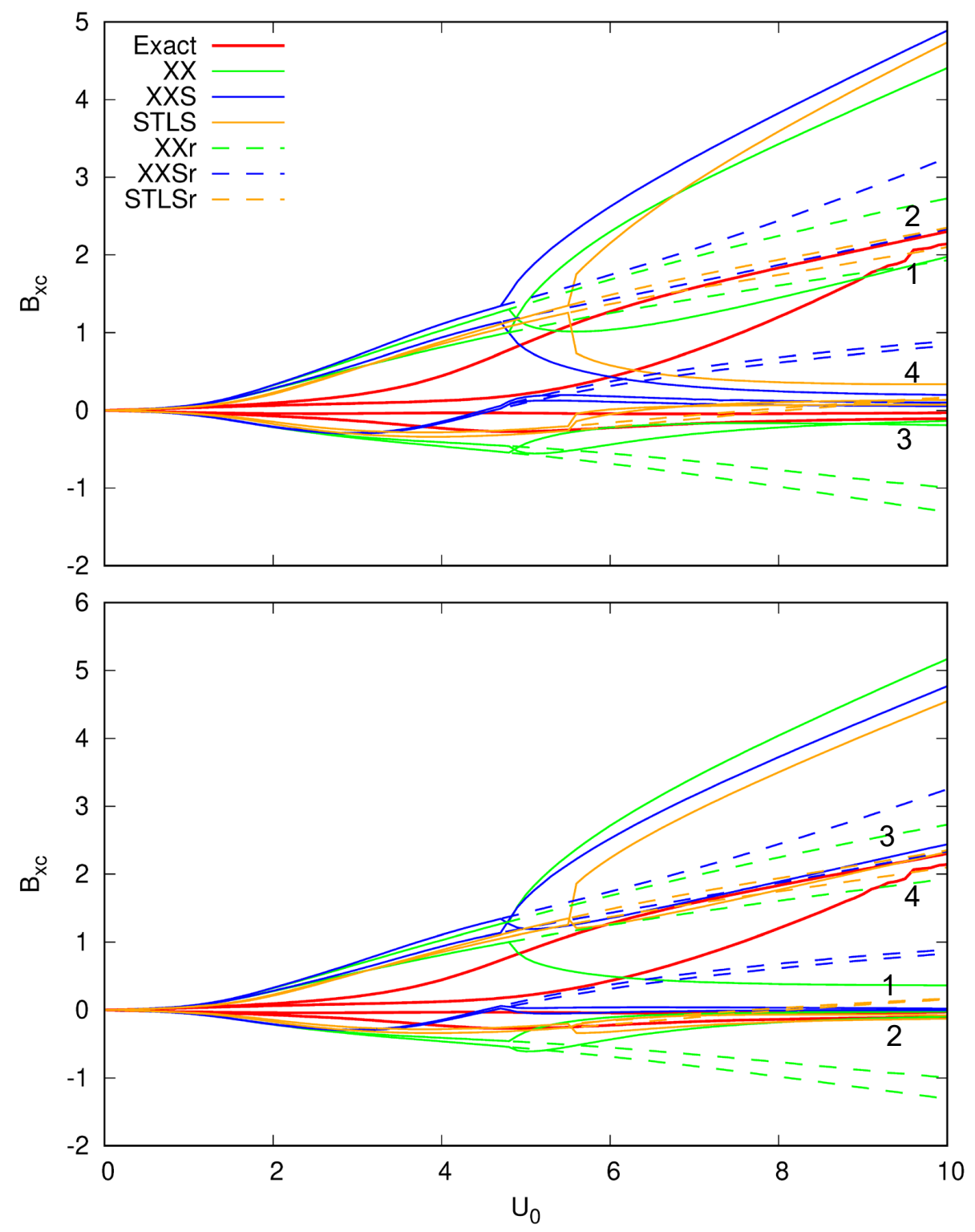

Figure 4.13: $x$ - (top) and $z$-component (bottom) of the xc magnetic field of the $C_{2}$-symmetric Hubbard tetramer. The numbers indicate lattice sites. 


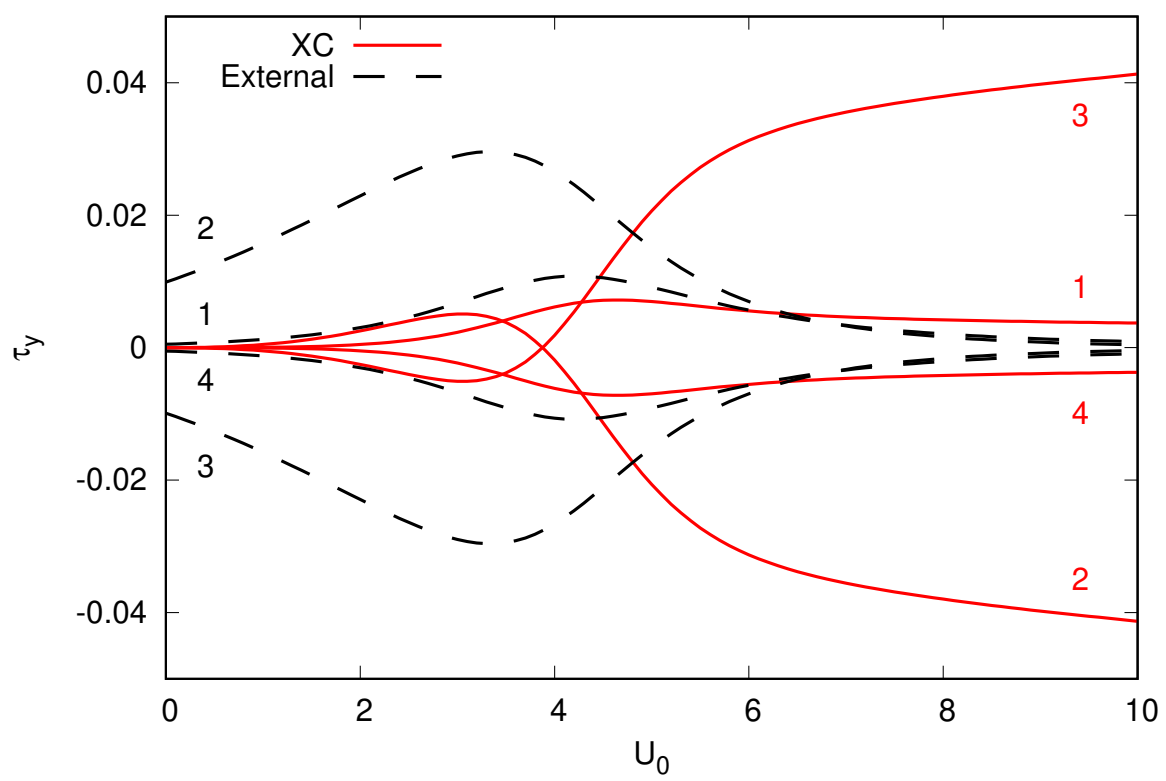

Figure 4.14: Comparison of xc torque $\boldsymbol{\tau}_{\mathrm{xc}, k}$ (red, full lines) and external torque $\tau_{\text {ext }, k}$ (black, dashed lines) of the $C_{2}$-symmetric tetramer. All torques only have a $y$-component. 

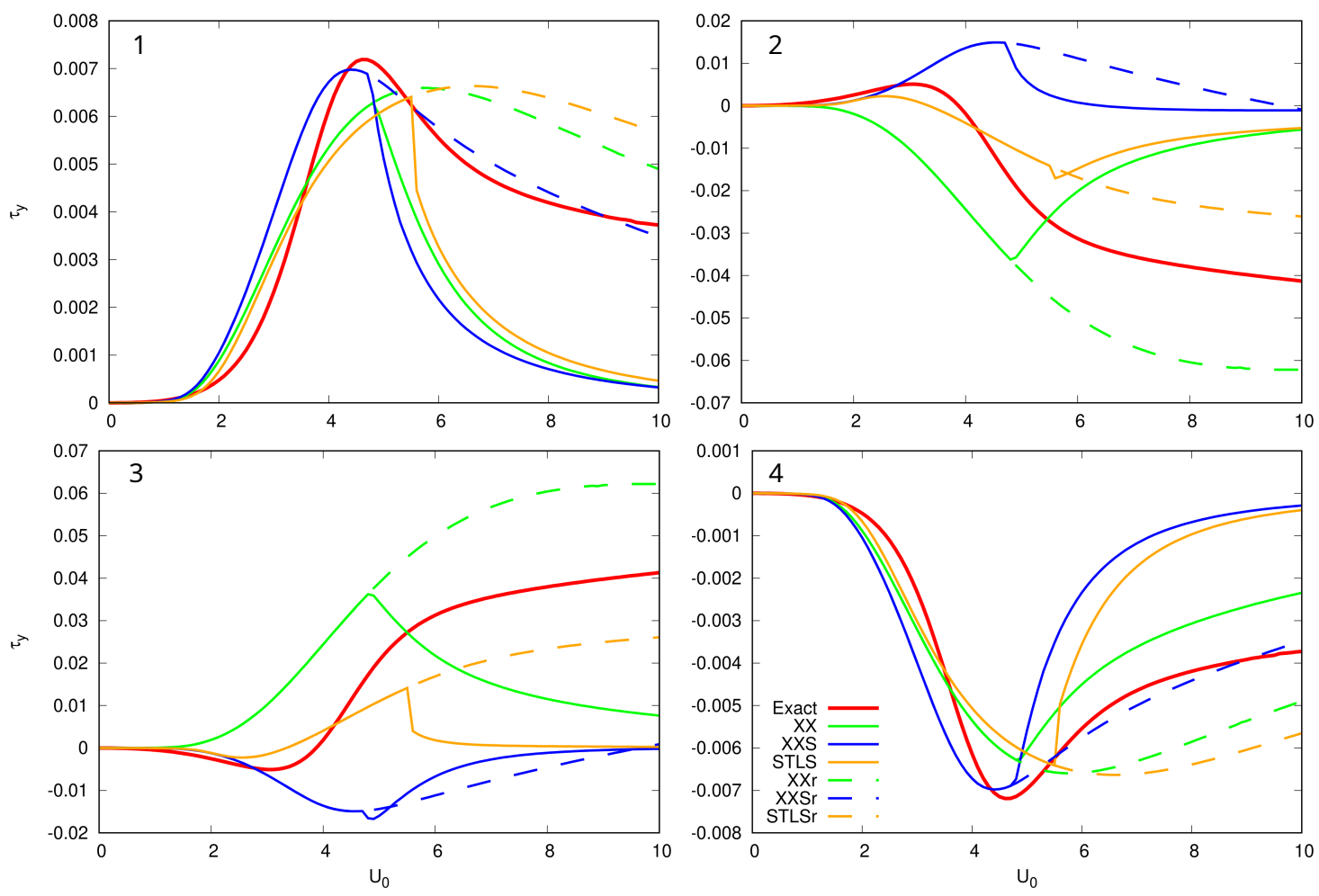

Figure 4.15: Comparison of exact xc torque $\boldsymbol{\tau}_{\mathrm{xc}, k}$ and unrestricted and restricted xc torque approximations for the $C_{2}$-symmetric tetramer, on the four lattice sites. All torques only have a $y$-component. 

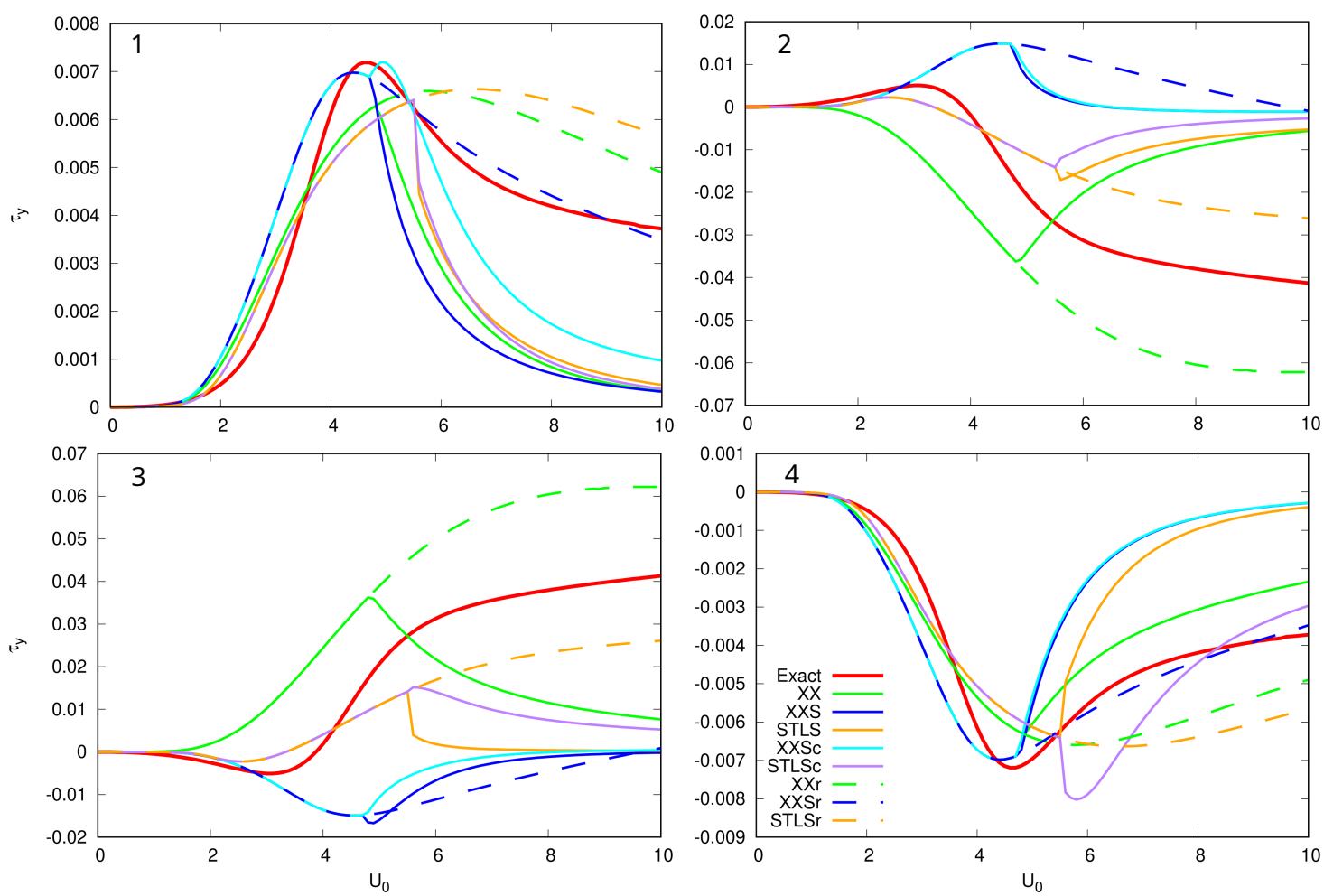

Figure 4.16: $\tau_{\mathrm{xc}}$ 's for $C_{2}$ symmetric system with torque corrections. Torque corrections are only required after the system spontaneously breaks symmetry, as the solutions satisfy the zero-torque theorem prior to breaking symmetry. 


\subsubsection{Ground-state energy, density and magnetization: break- ing and enforcing symmetry}

Figure 4.10 shows the ground-state energy $E_{\mathrm{gs}}$ for the $C_{2}$-symmetric Hubbard tetramer for interaction strengths $U_{0}$ between 0 and 10. The behavior is similar to the nonsymmetric case discussed earlier. The exact energy starts out at $E_{\mathrm{gs}}=-3.203$ for the noninteracting case, reaches the value $E_{\mathrm{gs}}=-0.603$ for $U_{0}=10$, and approaches a limiting value of $E_{\mathrm{gs}}=-0.4247$ for $U_{0} \rightarrow \infty$. As before, the crossover between the weakly and strongly correlated regimes occurs around $U_{0} \sim 4$.

The agreement between the exact energy and the (unrestricted) SDFT results is generally good for weak and moderate interactions strengths, but a significant ( $25 \%)$ deviation appears for large $U_{0}$. The inset to Fig. 4.10 shows the difference between approximate and exact ground-state energies. Overall, STLS performs the best; in the limit of large $U_{0}$, XX merges with STLS.

Each approximation reaches a critical $U_{0}$ where it is advantageous for the system to break $C_{2}$ symmetry to reduce the total energy of the system. This does not come as a surprise $[105,111,123]$ : unrestricted Kohn-Sham calculations with approximate xc functionals have the general tendency to break symmetries (spatial and/or spin) to lower the energy. This is known as the symmetry dilemma of DFT. The dashed lines in Fig. 4.10 are the restricted SDFT ground-state energies, obtained by enforcing the $C_{2}$ symmetry. The restricted energies keep increasing with $U_{0}$, in contrast with the exact $E_{\mathrm{gs}}$ and with the unrestricted (symmetry-broken) Kohn-Sham $E_{\mathrm{gs}}$. However, in spite of the overall improvement, the symmetry-broken solution still has a sizable energy error; recall that in the nonsymmetric case no symmetry breaking was needed,

and the agreement between the exact and the approximate Kohn-Sham $E_{\mathrm{gs}}$ was much 
better (see Fig. 4.3).

Figure 4.11 shows the density $n_{k}$ (top panel) and the $x$ - and $z$-components of the magnetization, $m_{x, k}$ and $m_{z, k}$ (middle and bottom panels). At $U_{0}=0$, the density is almost entirely concentrated at points 2 and 3, like in the nonsymmetric case (see the top panel of Fig. 4.4). However, as $U_{0}$ increases, differences between the symmetric and nonsymmetric tetramers start to appear: for strong correlations, the exact density becomes almost evenly distributed over the four lattice points, rather than accumulating on two points as in the nonsymmetric lattice. This is because the two configurations where the density localizes on points 1 and 3 and on points 2 and 4 are degenerate, since the magnetic field has equal strength on all points.

After breaking the symmetry, the unrestricted approximate SDFT calculations dramatically over-localize the density in the strongly correlated limit: the density is almost completely concentrated on points 2 and 4 . The symmetry breaking occurs at $U_{0}=4.72$ and $U_{0}=4.78$ for XXS and XX, respectively, and at $U_{0}=5.24$ for STLS.

For the exact magnetization (middle and bottom panels of Fig. 4.11, and right half of Table 4.1), the trends are very similar to the nonsymmetric case. At $U_{0}=0$, the system is only very weakly magnetized, with an exact wave function that has $99 \%$ singlet character. At $U_{0}=10$, the system is fully magnetized, and the exact wave function has $74 \%$ triplet character. As before, the exact magnetization vector is (anti)aligned with the applied magnetic field on each lattice site, which indicates a local spin quantization axis.

Unrestricted SDFT, on the other hand, produces a very different magnetization after the $C_{2}$ symmetry is broken. As we discussed above, the unrestricted density localizes on points 2 and 4 , and we see that $m_{x, 2}$ and $m_{z, 4}$ approach -1 . As for the 
exact case, the total magnetization adds up to 2 in the $U_{0} \rightarrow \infty$ limit, in spite of the wrong overall distribution.

The dashed lines in Fig. 4.11 show the density and magnetization resulting from symmetry-restricted SDFT. Not surprisingly, the agreement with the exact results is much better, although there are still some deviations. However, the price to pay is a wrong behavior of the total energy, as we saw above in Fig. 4.10.

\subsection{2 xc potentials, magnetic fields, and torques}

Figure 4.12 shows the exact Hartree potential and the exact xc potential on each lattice site, as a function of $U_{0}$. The $x$ - and $z$-components of the xc magnetic fields are shown in Fig. 4.13. The interpretation of these results is a bit more straightforward than for the nonsymmetric case discussed earlier (see Figs. 4.5 and 4.6). The Hartree potential approaches values close to 1 on sites 2 and 3 and close to -1 on

sites 1 and 4, which compensates the external potential. The xc potential is small compared to the Hartree potential (the fact that it approaches zero for $U_{0}=10$ seems accidental). Together, the Kohn-Sham potential is close to uniform, which explains the almost uniform density distribution in the strongly interacting limit, see Table 4.1. The strong xc magnetic fields, on the other hand, are responsible for almost fully magnetizing the system, similar to what we discussed earlier for the nonsymmetric case.

As before for the nonsymmetric case, the XX, XXS and STLS approximations for $V_{\mathrm{xc}, k}$ and $\mathbf{B}_{\mathrm{xc}, k}$ deviate significantly from the exact results, especially after the symmetry breaking. The agreement within the restricted-symmetry formalism is somewhat better. 
Figure 4.14 shows a comparison of the exact $\boldsymbol{\tau}_{\mathrm{xc}, k}$ (full lines) for the $C_{2}$-symmetric tetramer with the torques caused by the externally applied magnetic field, $\boldsymbol{\tau}_{\text {ext }, k}$ (dashed lines). As in the nonsymmetric case (Fig. 4.7), the external torque dominates over the xc torque for weak interactions. However, in the strongly correlated regime, the xc torques are now considerably larger (in magnitude as well as relative to the external torques) than in the nonsymmetric case.

The xc torques obtained with XX(r), XXS(r) and STLS(r) are presented in Figure 4.15. For small to moderate $U_{0}$ the agreement with the exact xc torques is decent, especially on sites 1 and 4 . The deviations on site 2 and 3 are more significant, however. As expected, the approximate xc torques fail completely for the unrestricted calculations after symmetry breaking. The restricted calculations (dashed lines) perform somewhat better in general. The excellent agreement between XXSr and the exact results on sites 1 and 4 seems, however, fortuitous. STLSr is most successful at capturing the general trends of the xc torques in both the weakly and strongly correlated regimes, mainly because it produces overall the best magnetizations, as seen in Figure 4.11.

Figure 4.16 shows the xc torques on the four sites the $C_{2}$-symmetric lattice. In addition to the results shown in Figure 4.15, we also include the torque-corrected results. The zero-torque theorem is satisfied for the symmetry-restricted case, so the torque-corrected results are only relevant once the $C_{2}$-symmetry is broken. Some differences between the XXS and XXSc results and between the STLS and STLSc results can be observed, but the xc torque correction has no impact on the symmetry breaking.

Next, to assess the significance of the xc torques, we have performed self-consistent 

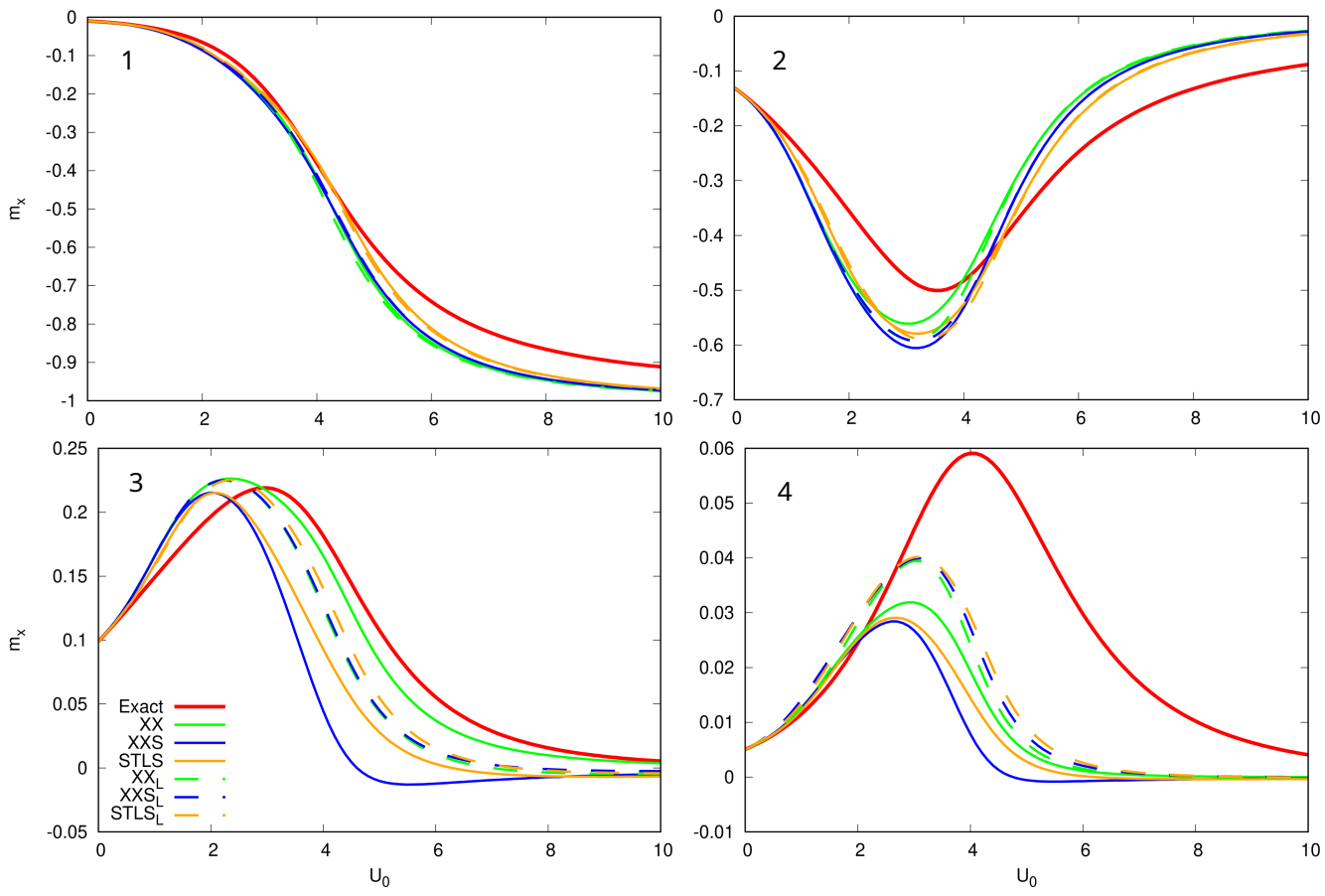

Figure 4.17: $x$-component of the magnetization for the nonsymmetric lattice, comparing exact results, Kohn-Sham results with the full $\mathbf{B}_{\mathrm{xc}}$, and Kohn-Sham results with the longitudinal $\mathbf{B}_{\mathrm{xc}}$ (dashed lines). 

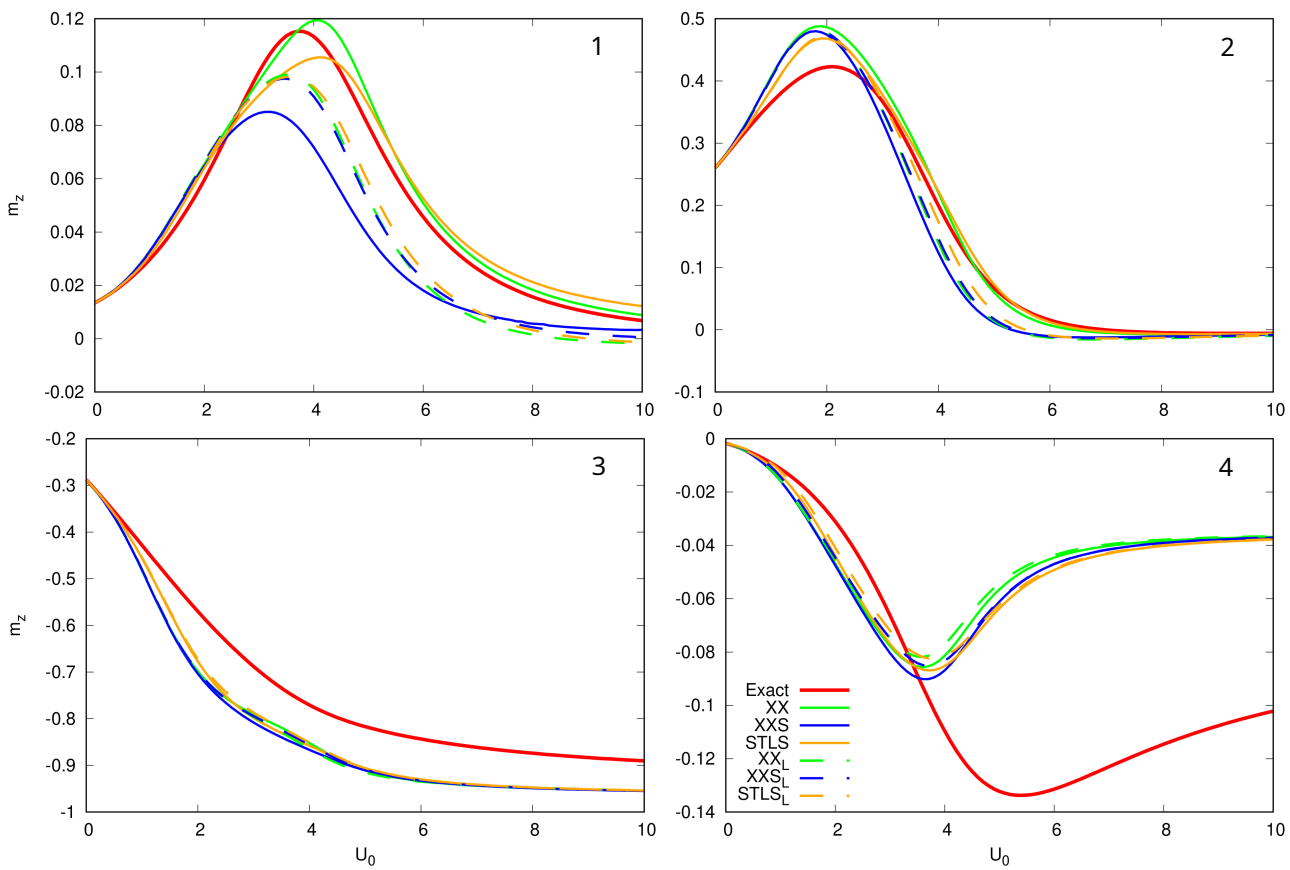

Figure 4.18: Same as Fig. 4.17, but for $m_{z, k}$.
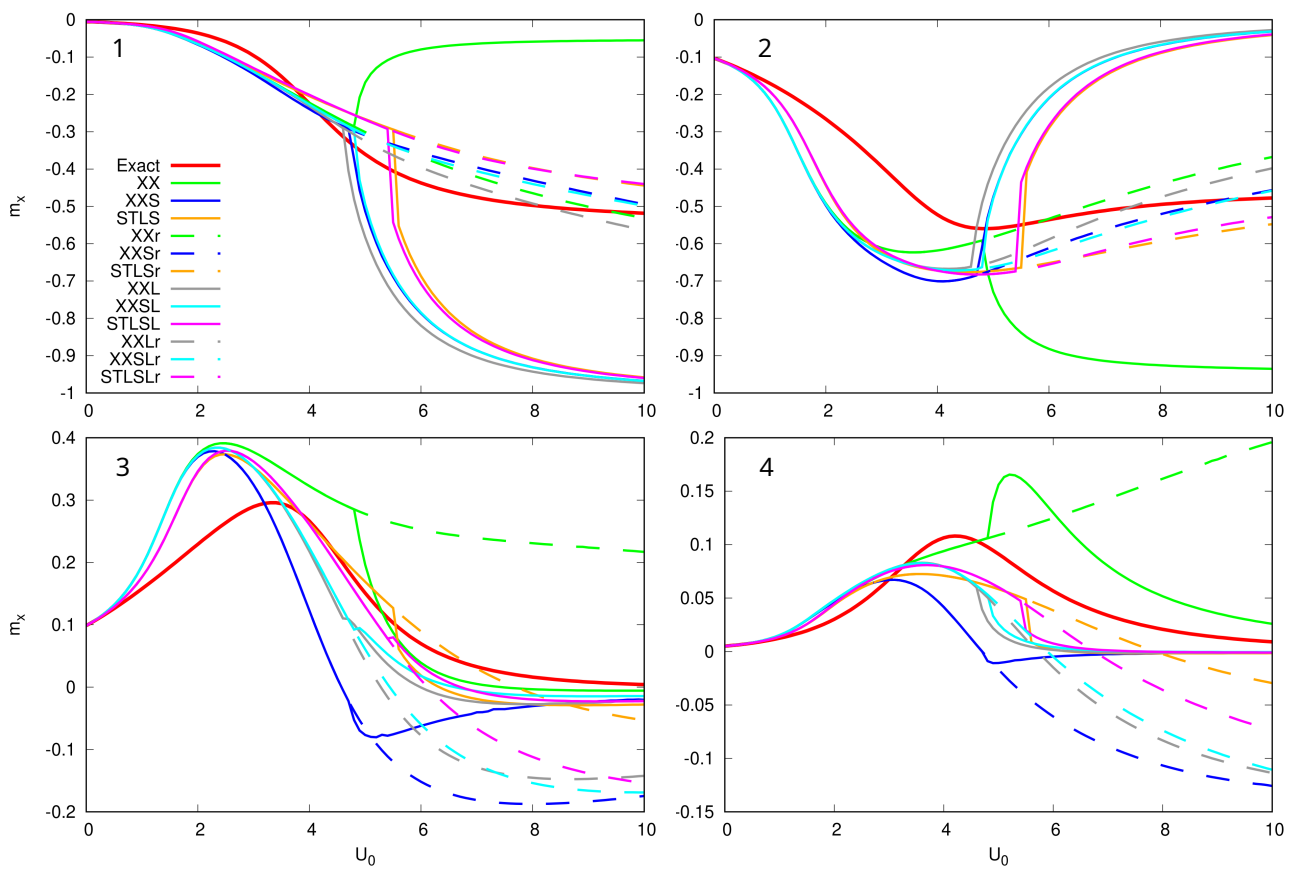

Figure 4.19: Same as Fig. 4.17, but for the $C_{2}$-symmetric lattice. 

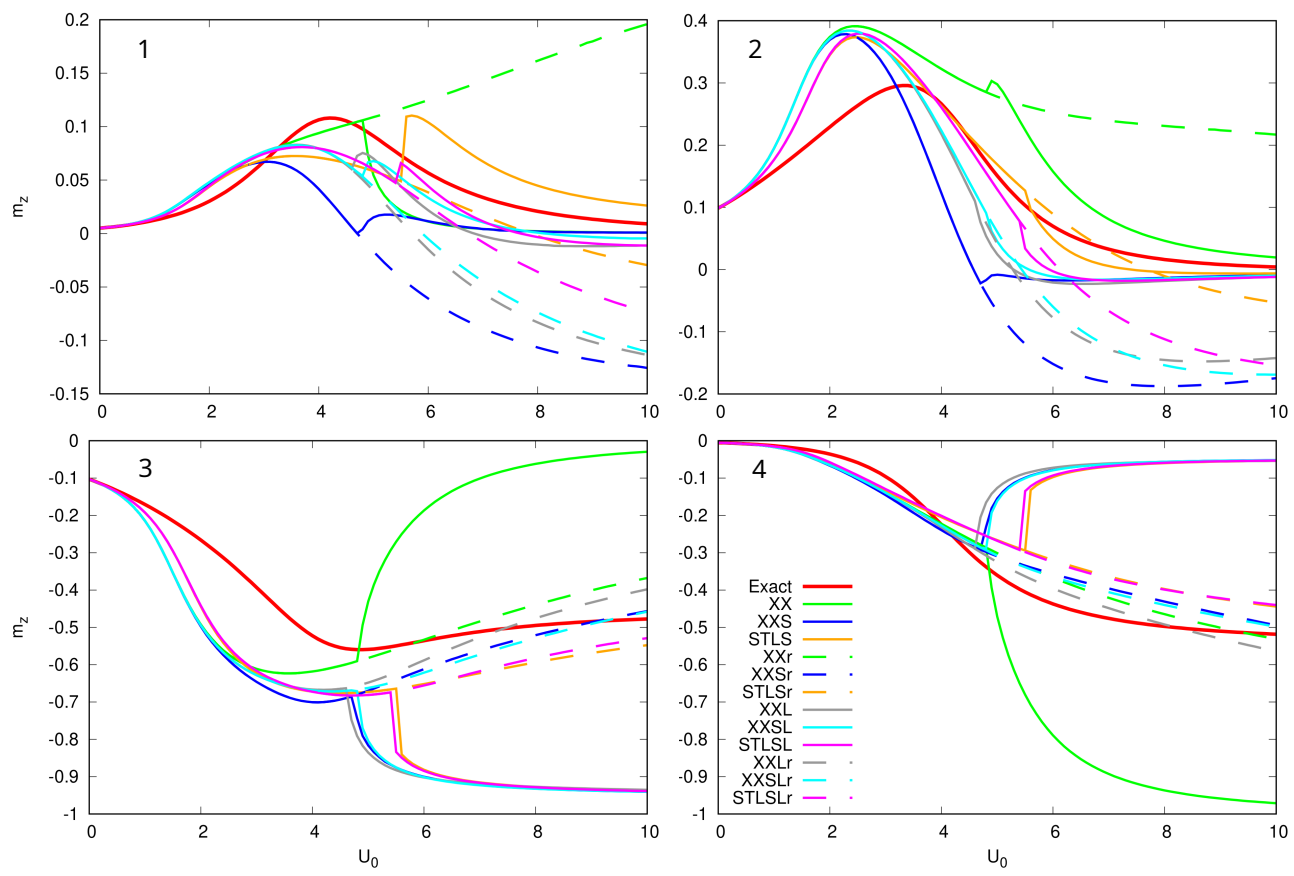

Figure 4.20: Same as Fig. 4.18, but for the $C_{2}$-symmetric lattice.

calculations in which only the longitudinal component of the xc magnetic field is included, defined as

$$
\mathbf{B}_{\mathrm{xc}, \|}=\frac{\left(\mathbf{m} \cdot \mathbf{B}_{\mathrm{xc}}\right) \mathbf{m}}{m^{2}}
$$

Figures 4.17 and 4.18 show the $x$ - and $z$-components of the magnetization of the nonsymmetric tetramer, and Figs. 4.19 and 4.20 show the same for the $C_{2}$-symmetric tetramer, comparing the exact results, the Kohn-Sham results with the full $\mathbf{B}_{\mathrm{xc}}$, and the Kohn-Sham results with the longitudinal $\mathbf{B}_{\mathrm{xc}}$ (dashed lines).

Overall, the differences between the full calculations and the longitudinal-only calculations are mostly minor, except for XX, see below. In the nonsymmetric case, the biggest relative deviations are on sites 3 and 4 for the $x$-component and on site 1 for the $z$-component; however, on these sites, the respective components of 
magnetization are small to begin with.

The same is the case for the $C_{2}$-symmetric lattice, at least for XXS and STLS. For $\mathrm{XX}$, on the other hand, the longitudinal restriction causes some major differences for the unrestricted solution, and seems to push the symmetry breaking along opposite directions. Some differences, although less pronounced, also appear for the symmetryrestricted XX solutions, which now are closer to the XXS and STLS solutions. It is not obvious why the XX appears to be more sensitive to the presence of transverse magnetic fields, but it is clearly related to the fact that XX produces larger xc torques than XXS and STLS.

As for the nonsymmetric lattice, the differences are only relatively minor, except for XX: the XX functional seems to be more sensitive to the presence of the transverse xc magnetic field, which affects the way in which the symmetry is broken. Overall, the longitudinal-only XX is closer to its XXS and STLS counterparts (and to the exact solution) than when the full $\mathbf{B}_{\mathrm{xc}, k}$ is used, which would suggest that $\mathrm{XX}$ does not describe transverse xc effects very well. This is consistent with the behavior of the xc torques shown in Fig. 4.15 (and, to some extent, also in Fig. 4.8), which shows that the XX torques tend to be somewhat exaggerated. The behavior of XXS and STLS comes across as more robust.

\subsection{Conclusions}

In this chapter we have carried out a detailed computational study of a simple model system: two interacting electrons on a four-site extended Hubbard lattice, in the

presence of noncollinear magnetic fields. Our goal was to find out how a Kohn-Sham 
system, through its xc scalar potentials and magnetic fields, manages to reproduce the exact ground-state density and magnetization of the interacting system, particularly for situations where the interaction is strong. We discovered that the xc magnetic field plays a key role in ensuring that the magnetic behavior of strongly correlated, localized electrons is determined by local spin quantization axes on each lattice site.

We compared the exact xc potentials and magnetic fields with several orbitaldependent approximations, and found that their performance is overall quite good, but depends on whether the system has any symmetries. In the case where the Hubbard lattice has $C_{2}$-symmetry, we found that the approximate Kohn-Sham calculations tend to break this symmetry once the interaction strength becomes sufficiently large, thus lowering the energy. This, of course, is no surprise: the so-called symmetry dilemma is a well-known side effect of using approximate xc functionals in SDFT, which originates from a failure to describe static correlation [124] and, concurrently, violations of the fractional spin constancy condition $[125,126]$. The STLS approach captured some of these correlation effects and performed a bit better than the exchange-only functionals; a more complete cure of the symmetry dilemma may be achieved via higher-level orbital functionals based on the adiabatic-connection fluctuation-dissipation theorem $[124,127]$.

A main outcome of this study is that the xc torques seem to have a relatively minor importance for the ground-state energy, density and magnetization, compared to the xc potentials and longitudinal xc magnetic fields - at least for the simple model systems considered here. This suggests that the widely used standard approximations of noncollinear magnetism (which have no transverse magnetic xc contributions) should be adequate in most situations, as long as they reproduce the longitudinal xc magnetic 
fields sufficiently accurately.

However, our study did not include spin-orbit coupling, which is an important aspect of noncollinear magnetism in many materials. In particular, we needed to enforce the noncollinear magnetic state via applied magnetic fields, whereas effects such as canted or frustrated spins arise naturally without any applied fields. Such systems are often characterized by a competition between degenerate or nearly degenerate states with different spin configurations, and it is likely that xc torque effects then play a much more important role. To study these kinds of situations, Hubbard models that include spin-orbit coupling are a promising approach.

A perhaps even more important question concerns the role of xc magnetic fields for spin dynamics. While the xc torques appear less crucial in the ground state, they may become more important as the magnetic system evolves in time, following a sudden switching or a pulsed excitation. Such studies, again based on simple Hubbard-type systems, are currently in progress.

Lastly, while simple lattice models serve as important benchmarks for SDFT and can produce a wealth of new physical insight, it will be important to investigate the performance of orbital-dependent xc functionals for noncollinear magnetic systems in real space. The XXS approximation will be very well suited for this purpose due to its simplicity and reasonable accuracy. The STLS approach was found to perform well for the model systems considered here (as long as correlations are not too strong), but would be numerically much more expensive for real-space systems. Finding orbitaldependent correlation functionals for noncollinear magnetic materials, at a reasonable computational cost, remains an ongoing challenge. 


\section{Chapter 5}

\section{Spin-orbit coupling and exchange-correlation torque effects in real-time spin-density-functional theory}

\section{$5.1 \quad$ Introduction}

Spin effects and the effect of the exchange-correlation (xc) field have been explored previously for the Hubbard dimer and generalized expansions for additional lattice sites $[105,106]$. In that preceding work, it was shown that there exist regimes where the xc fields are on the order of magnitude as the external applied field [81]. Using exact diagonalization and inversion of the Kohn-Sham (KS) equations, one can solve for the full xc field effects in any situation for the Hubbard model.

Previous work only explored the effects with no spin-orbit coupling (SOC) term and for the time-independent case only. In this final chapter, we focus on two general- 
ized Hubbard systems: the Hubbard tetramer that was explored in the previous chapter and the Hubbard trimer with periodic boundary conditions. With the Hubbard tetramer system, we continue to focus on xc-torques and their effects when they are included in Kohn-Sham solutions. As we can continue to solve the Schrödinger equation exactly for the tetramer system, we can propagate both the exact Schrödinger and KS solutions and compare the spin-dynamics of our system accordingly. To simplify the system, we leave out any SOC to focus on xc-torque and functional approximation effects.

We are still interested in how SOC plays a role in the rich physics of our Hubbard system, so we move to a trimer system with periodic boundary conditions. As discussed in [105], the trimer system, especially when in a periodic configuration (i.e., a triangular lattice), has no xc-torque in the exchange-only approximation, due to the symmetric nature of the lattice. This was the primary reason for exploring the tetramer (including nearest-neighbor interactions), as it lifted the symmetry of the lattice sites, allowing for rich xc-torque effects. In this case, we have allowed ourselves to remove some of the complexity of the xc-torques, while enabling us to use the same Slater potential approximation with the inclusion of SOC. Importantly, the inclusion of SOC terms allows us to prepare our lattice in a noncollinear spin configuration where the spins on each lattice site are oriented into or out of the triangle. Additionally, by tuning the kinetic (hopping) and SOC term, we can explore a rich physical phase space with two very well defined collinear and noncollinear phases.

In 5.2, we will cover the theoretical background of our periodic trimer model and denote some of the differences between our trimer model and the tetramer model in Chapter 4. While discussing the theoretical differences between the trimer and 
tetramer, we examine how adjustments to the SOC and kinetic (hopping) coefficients give rise to a phase diagram with two robust phases. Once we discuss the phase diagram, we will explore how the system evolves under the perturbation of a magnetic field. Importantly, this noncollinear model can be easily extrapolated into real physically relevant systems, such as spin ices or frustrated spin lattices [128].

After we explore the trimer system, in Section 5.3 we will return to the Hubbard tetramer and continue exploring the real-time evolution of the electronic spins. Returning to the tetramer, we will also refocus our attention on the xc-torques that are present when using the Slater approximation with nearest-neighbor interactions. We will end with our final conclusions in 5.4, highlighting the interesting emergent dynamics that come from inclusion of the xc-torques and SOC coefficients.

\subsection{Hubbard Trimer}

In the previous chapter we explored a generalized Hubbard model, primarily focusing on a four site, non-periodic lattice filled with two electrons. Here, we can use the same starting point for our exploration, equations 4.1 and 4.2 from Chapter 4 , where we use half-filling on an equilateral triangular lattice. This means that our system is now governed by periodic boundary conditions as the electron density on each site now has allowable jumps into two sites. 


\subsubsection{Theoretical Background}

Similarly to our previous example, we can rewrite the spin Hamiltonian within the complete singlet-triplet basis, giving us

$$
\begin{aligned}
& \hat{H}_{\text {lat }}=-t \sum_{\langle k, l\rangle \sigma}\left(\hat{c}_{k \sigma}^{\dagger} \hat{c}_{l \sigma}+\hat{c}_{l \sigma}^{\dagger} \hat{c}_{k \sigma}\right)+U_{0} \sum_{k} \hat{c}_{k \uparrow}^{\dagger} \hat{c}_{k \uparrow} \hat{c}_{k \downarrow}^{\dagger} \hat{c}_{k \downarrow} \\
& +\sum_{k}\left[V_{k} \hat{\mathbf{c}}_{k}^{\dagger} \hat{\mathbf{c}}_{k}+\mathbf{B}_{k} \cdot \hat{\mathbf{c}}_{k}^{\dagger} \boldsymbol{\sigma} \hat{\mathbf{c}}_{k}\right] .
\end{aligned}
$$

We notice in Eq. (5.1) that we no longer have any nearest-neighbor interaction, in contrast with Eq. (4.5). Instead, for the trimer, we will add in a SOC term that is defined within our second-quantization formalism as

$$
\hat{H}_{\mathrm{SO}}=i C \sum_{i \neq j} \sum \sigma \sigma^{\prime} \hat{c}_{i \sigma}^{\dagger}\left[\mathbf{f}_{i j} \cdot \sigma\right]_{\sigma \sigma^{\prime}} \hat{c}_{j \sigma^{\prime}}
$$

where $C \in \mathbb{R}$ is the SOC interaction coefficient, which can take arbitrary values $\geq 0$, and $\mathbf{f}_{i j}$ is a unit vector that denotes the direction of the SOC term $[116,129]$. For a system with $D_{3 h}$ symmetry, otherwise known as a planar triangular lattice system, Moriya rules enforce that $\mathbf{f}_{12}=\mathbf{f}_{23}=\mathbf{f}_{31}=\mathbf{z}$, leaving us with only the $z$-component in Eq. (5.2). Explicitly, this gives for the trimer

$$
\begin{aligned}
\hat{H}_{\mathrm{SO}} & =i C\left\{\left[\hat{c}_{1 \uparrow}^{\dagger} \hat{c}_{2 \uparrow}-\hat{c}_{1 \downarrow}^{\dagger} \hat{c}_{2 \downarrow}\right]+\left[\hat{c}_{2 \uparrow}^{\dagger} \hat{c}_{3 \uparrow}-\hat{c}_{2 \downarrow}^{\dagger} \hat{c}_{3 \downarrow}\right]+\left[\hat{c}_{3 \uparrow}^{\dagger} \hat{c}_{1 \uparrow}-\hat{c}_{3 \downarrow}^{\dagger} \hat{c}_{1 \downarrow}\right]\right\} \\
& -i C\left\{\left[\hat{c}_{2 \uparrow}^{\dagger} \hat{c}_{1 \uparrow}-\hat{c}_{2 \downarrow}^{\dagger} \hat{c}_{1 \downarrow}\right]+\left[\hat{c}_{3 \uparrow}^{\dagger} \hat{c}_{2 \uparrow}-\hat{c}_{3 \downarrow}^{\dagger} \hat{c}_{2 \downarrow}\right]+\left[\hat{c}_{1 \uparrow}^{\dagger} \hat{c}_{3 \uparrow}-\hat{c}_{1 \downarrow}^{\dagger} \hat{c}_{3 \downarrow}\right]\right\}
\end{aligned}
$$

which shows that the SOC terms can be viewed as purely imaginary hopping terms. 
This leaves us in total with our full Hubbard Hamiltonian, with SOC, as

$$
\begin{aligned}
& \hat{H}_{\text {total }}=-t \sum_{\langle k, l\rangle \sigma}\left(\hat{c}_{k \sigma}^{\dagger} \hat{c}_{l \sigma}+\hat{c}_{l \sigma}^{\dagger} \hat{c}_{k \sigma}\right)+U_{0} \sum_{k} \hat{c}_{k \uparrow}^{\dagger} \hat{c}_{k \uparrow} \hat{c}_{k \downarrow}^{\dagger} \hat{c}_{k \downarrow} \\
& \quad+i C \sum_{i \neq j} \sum \sigma \sigma^{\prime} \hat{c}_{i \sigma}^{\dagger}\left[\mathbf{f}_{i j} \cdot \sigma\right]_{\sigma \sigma^{\prime}} \hat{c}_{j \sigma^{\prime}}+\sum_{k}\left[V_{k} \hat{\mathbf{c}}_{k}^{\dagger} \hat{\mathbf{c}}_{k}+\mathbf{B}_{k} \cdot \hat{\mathbf{c}}_{k}^{\dagger} \boldsymbol{\sigma} \hat{\mathbf{c}}_{k}\right] .
\end{aligned}
$$

As with the four site Hubbard system, we again have the KS equation

$$
\left[\left(-\frac{\nabla^{2}}{2}+V_{\mathrm{KS}}(\mathbf{r})\right) I+\boldsymbol{\sigma} \cdot \mathbf{B}_{\mathrm{KS}}(\mathbf{r})\right] \Psi_{i}(\mathbf{r})=\epsilon_{i} \Psi_{i}(\mathbf{r})
$$

where $I$ is a $2 \times 2$ identity matrix and $\sigma$ denotes the spin state of the particle. Recall that in Chapter 4, we discussed that most calculations done when using noncollinear spins rely on the local spin-density approximation (LSDA) which causes the local xc magnetic field to be parallel to the local magnetization at all points. Doing so satisfies Eq. (4.4), the so-called zero torque theorem, but the xc fields can have perpendicular components when using functionals that do not rely on the LSDA. However, this does not mean that all local torques must be zero. To the contrary, local torques can be nonzero so long as the total xc torque is equivalent to zero.

In this chapter, we use the Slater approximation to the noncollinear Optimized Effective Potential (OEP) [105]. The Slater potential for noncollinear spins, $\underline{\underline{v}}^{\mathrm{x}}(\mathbf{r})$, is defined as follows:

$$
\underline{\underline{n}} \underline{\underline{v}}^{\mathrm{xS}}+\underline{\underline{v}}^{\mathrm{xS}} \underline{\underline{n}}=\underline{\underline{b}}
$$

where the right-hand side $\underline{\underline{b}}$ is given by

$$
\underline{\underline{b}}(\mathbf{r})=-2 \int \frac{d \mathbf{r}^{\prime}}{\left|\mathbf{r}-\mathbf{r}^{\prime}\right|} \underline{\underline{\gamma}}\left(\mathbf{r}, \mathbf{r}^{\prime}\right) \underline{\underline{\gamma}}\left(\mathbf{r}^{\prime}, \mathbf{r}\right)
$$




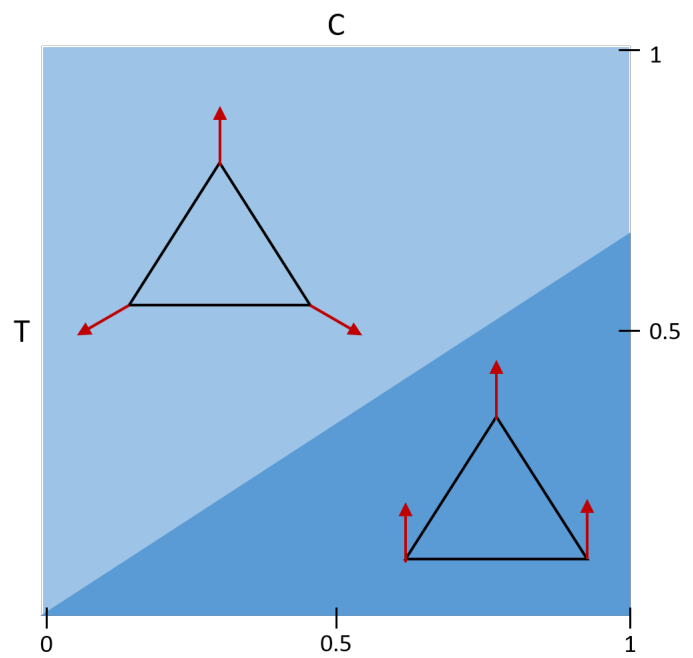

Figure 5.1: Kohn-Sham phase diagram for the Hubbard trimer with a small in-plane magnetic field on two lattice sites to lift the degeneracy of the system. The phase diagram found here is nearly identical to what was published in [117].

Here, $\gamma_{\sigma \sigma}\left(\mathbf{r}, \mathbf{r}^{\prime}\right)$ is the spin-resolved reduced one-particle KS density matrix:

$$
\gamma_{\sigma \sigma^{\prime}}\left(\mathbf{r}, \mathbf{r}^{\prime}\right)=\sum_{j}^{N} \psi_{j \sigma}(\mathbf{r}) \psi_{j \sigma^{\prime}}^{*}\left(\mathbf{r}^{\prime}\right)
$$

By using the Slater potential, we no longer trivially satisfy the zero-torque theorem through a local spin density approximation. For the case of the trimer with only on-site interactions, the symmetry of the system prohibits non-zero xc spin torques, allowing us to focus on the effects of the inclusion of the SOC term.

\subsubsection{Time-dependence}

Much of the underlying theory related to the ground-state was already covered and discussed in Chapter 4, so to save space and our readers' sanity, we will bypass revisiting the nuances of the ground state solutions. As we covered above though, we 


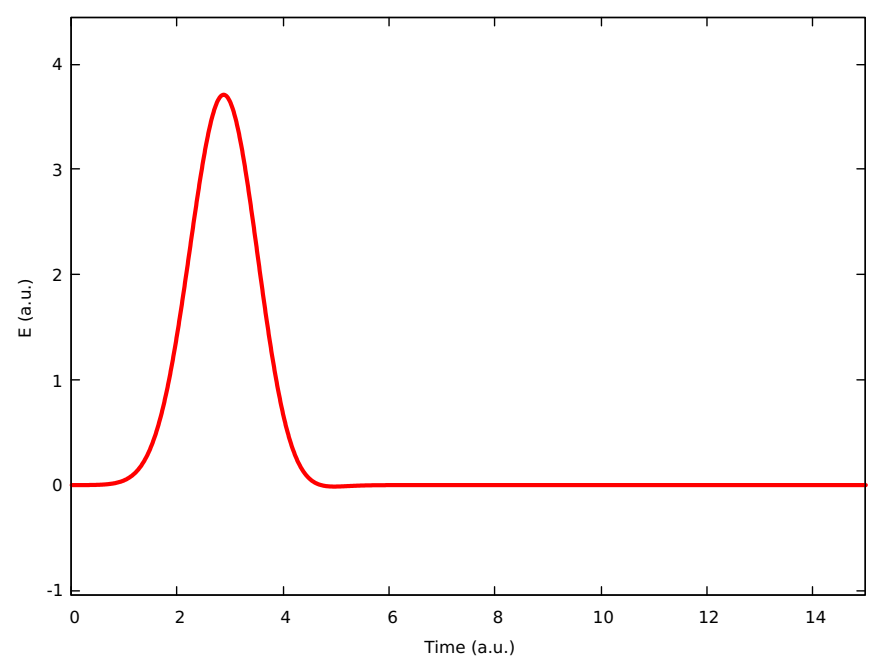

Figure 5.2: Time-dependent magnetic field applied to the trimer system. The field is a Gaussian pulse applied only in the $x$-direction.

have included SOC effects to both make our system more realistic and to allow for noncollinear magnetization in our system. Including SOC is essentially the introduction of a complex hopping term, which introduces spin effects that allow for the precession of electron spins on each lattice site. By adjusting the SOC and kinetic hopping terms, we are able to probe our system and explore the spin orientation phase space of our system. It is important to note that our system is highly degenerate, and in order to separate the degenerate states computationally, we must introduce a very small magnetic field to lift the degeneracy of the states. For the trimer, the magnetic field is on the order of $10^{-10}$ a.u. in the $x$ - and $y$-directions on sites 1 and 2 , respectively. The resultant trimer phases are extremely similar to the phase space plotted in [117], and the adapted phase diagram can be seen in Fig. 5.1

To capture the magnetization in real-time, we use real-time TDDFT, propagating the wavefunctions with the time operator, $\mathcal{U}(t)$, exciting our system with the magnetic pulse shown in Fig. 5.2. The pulse is applied only along the $x$-axis and hits each 
lattice site simultaneously. Unfortunately, simply applying our operator as an exponential function is numerically unstable. To get around this, numerous methods have been developed and some were touched upon in Chapter 2, Section 2.4.3. Here, we employ the Crank-Nicolson algorithm which gives a numerically stable solution. Explicitly, the Crank-Nicolson algorithm, written for our particular partial-differential equation, is defined as in Eq. (2.34). Rather than solving the partial-differential equation analytically, where we would then be applying our time operator as an exponential function, the Crank-Nicolson method takes the finite differences between our wavefunction and the evolved wavefunction at the next time step. To be more precise, the Crank-Nicolson method actually evaluates our time evolution at times $t-\frac{1}{2}$ and $t+\frac{1}{2}$, or the half-time steps.

By using this implicit differentiation method, we have a numerically stable solution over a broad selection of time step sizes that is accurate to the order of $(\Delta t)^{2}$. In addition to this, our solution method maintains the unitarity of the time propagation operator. Thus, the evolution of our wavefunctions will preserve normalization over the course of the time evolution.

Despite the wonderful qualities that are preserved by the Crank-Nicolson method, we now have to worry about our solution to our wavefunctions at the half time steps of our propagation space. The solution is to perform a predictor-corrector scheme to ensure our wavefunction is not succumbing to numerical instabilities arising from our selected time evolution method. To ensure this is the case, we calculate the wavefunction at our new time, $t$, and calculate the new density, $n$. We expect, given that our excitation will not ionize our system, that we should remain at a constant total number of electrons. Local density fluctuations are allowable in the same way 


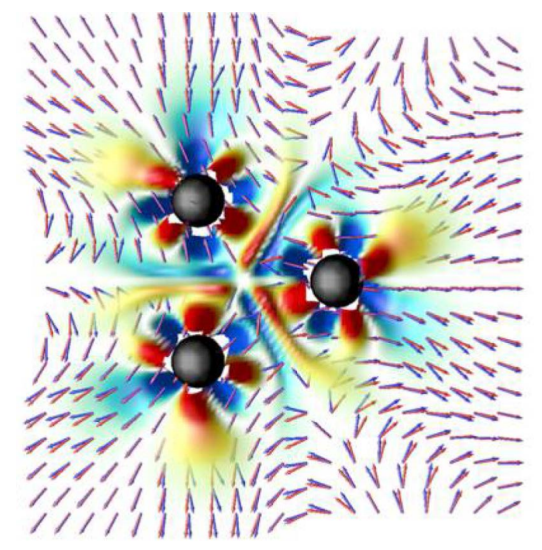

Figure 5.3: Chromium trimer showing the noncollinear spin configuration. Additionally, due to the approximation being used, there is an xc spin torque present in the trimer, seen by the fact that the red and blue arrows are not on top of each other. Adapted from [101].

that local magnetization and xc torques can occur, so long as the sum over all lattice sites correctly satisfies our physical requirements. Once global density convergence has been met, the next time step is approximated and the predictor-corrector scheme continues.

\subsubsection{Trimer results and discussion}

Applying the time-propagation methods and including SOC, we can examine how Kohn-Sham solutions near a highly degenerate phase boundary (see Fig. 5.1) behave once allowed to propagate in time, following a short-pulse excitation. The reason for exploring this Hubbard trimer phase space is to get a sense for how the easyto-compute KS potential approximations will behave in systems where different spin phases are present. One potential place of occurrence for this is a noncollinear spin system such as a Chromium trimer, as shown in Fig. 5.3, or a spin ice system like $\mathrm{Dy}_{2} \mathrm{Ti}_{2} \mathrm{O}_{7}[128]$. 
As can be clearly inferred from the phase diagram in Fig. 5.1, our trimer system has two primary parameters that can be tuned to explore their effect on the spin dynamics. We discussed already the SOC component, which we can control the strength of by varying the variable $C$ in Eq. (5.2). By setting $C=0$, we turn off the SOC, retrieving the behavior seen in Fig. 5.4, where the spins no longer precess after the passage of the magnetic pulse. It is interesting that the spins continue to have very small oscillations in the KS system, while the exact solution demonstrates that once the SOC component is removed, there is no effective mechanism that continues to cause the spins to oscillate.

We find from Fig. 5.4 that the magnetization along $x$ is not affected by the magnetic field pulse. There is some induced precession along $y$ and $z$, and after the end of the pulse, some finite magnetization along $z$ persists. This is qualitatively well reproduced by the TDDFT calculation.

The second parameter we can tune is the hopping term, $t$. Effectively, $t$ is the kinetic energy term for our trimer system. By setting $t \ll 1$, we effectively isolate each electron to a single lattice site, as the kinetic energy will not be high enough to allow the electrons to hop to a neighboring lattice site. As $t$ increases, electronic density becomes easier to transition between lattice sites.

By mapping the magnetic alignment phase space for the trimer system, we can clearly see in Fig. 5.1a that there are two unique magnetic phases. The first phase is a collinear spin phase where all the electronic spins are aligned, while the second phase is where the spins become noncollinear and lie within the plane, pointing outward from the triangular Hubbard lattice. By applying a small magnetic field, on the order of $10^{-10}$ on two sites that are in-plane (to reduce the degeneracy of the spin states), we 

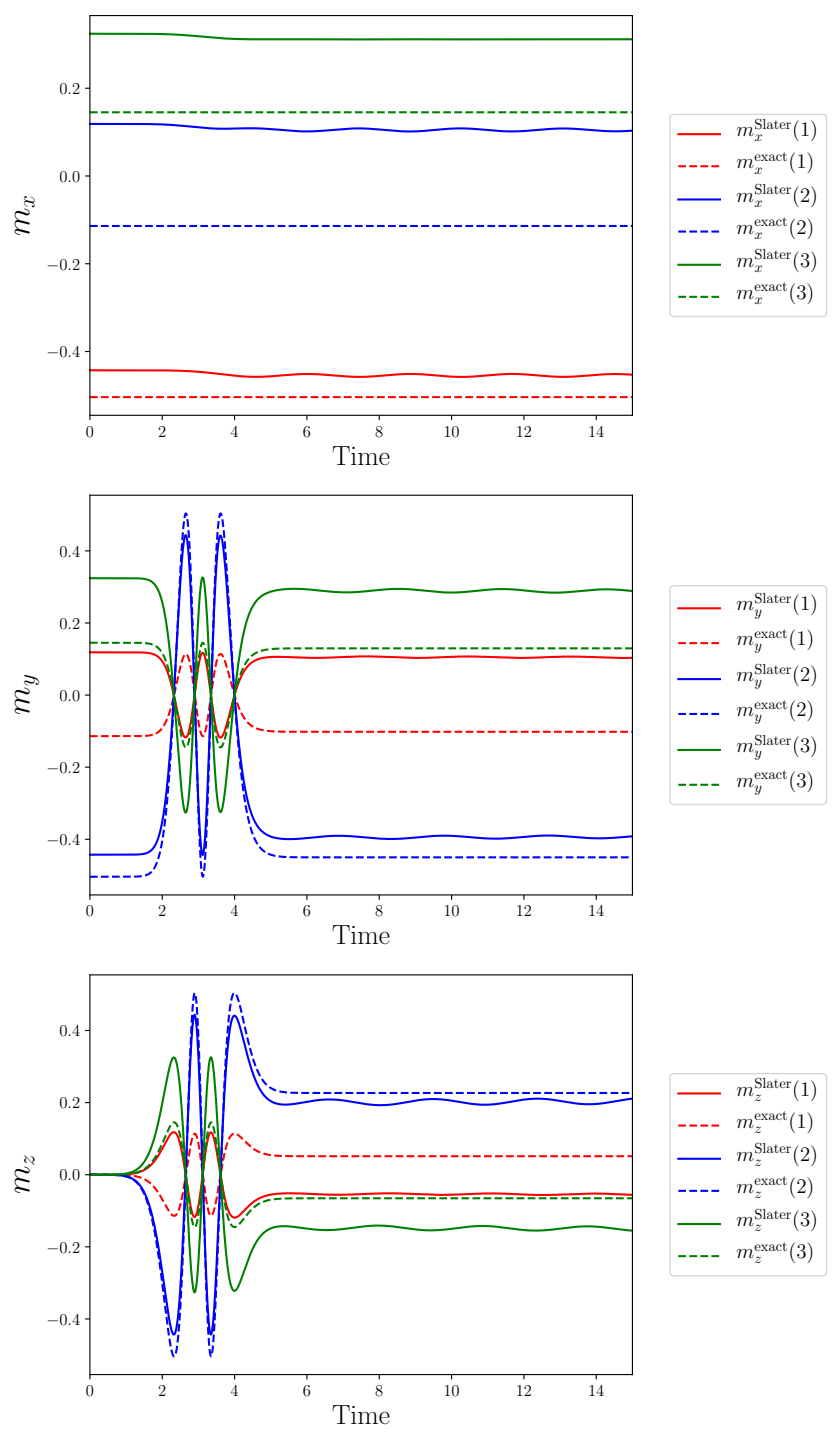

Figure 5.4: Real-time magnetization precession due to a magnetic pulse along the $x$-axis. The SOC has been set to zero and the hopping constant is set to 0.8 . The magnetizations react to the magnetic pulse which turns on just before $t=2$, but since there is no SOC to mediate the continued changing magnetic moment, the magnetizations remain static after the termination of the magnetic field. 
can prepare our ground state in the generalized Hartree-Fock configuration [117]. The Slater approximation of the OEP reduces to the Hartree-Fock solution, giving us a way of comparing the Hartree-Fock solution to our Kohn-Sham methods. Additionally, we also solve our system exactly for the same phase space and recover the same phase diagram seen in Fig. 5.1.

As in Chapter 4, we can define the magnetization as

$$
\mathbf{m}(\mathbf{r})=\sum_{i}^{N} \operatorname{tr}\left[\boldsymbol{\sigma} \Psi_{i}(\mathbf{r}) \Psi_{i}^{\dagger}(\mathbf{r})\right]
$$

Here, $\boldsymbol{\sigma}$ are the Pauli spin matrices. We notice that the wavefunctions, $\Psi_{i}(\mathbf{r})$, are dependent not only on position, but they are implicitly dependent upon $\mathbf{B}_{\mathrm{xc}}$ that are calculated self-consistently through the KS formalism. Recall that by using the Slater approximation, our $\mathbf{B}_{\mathrm{xc}}$ no longer trivially satisfy the zero torque theorem locally, but due to our configuration, we have no torques that will extraneously appear.

As can be seen in Fig. 5.1, if we carefully select our hopping and SOC coefficients we locate ourselves on the magnetic phase boundary of our trimer. By doing this, we essentially select a degenerate solution space, leaving us with a magnetization solution that tenuously holds to either region. Once our system is properly prepared at this boundary, we then kick the system with our magnetic pulse to attempt to determine the robustness of the spin state and examine how our magnetizations evolve near the boundary.

From Fig. 5.5, we can see that if we select a point in our phase space that is just under the phase boundary at $C=0.4$ and $t=0.2309$, we begin in a spin collinear state. This is easily seen by the fact that all three colored lines for both the KS Slater and exact solutions are on top of each other. Interestingly, when we propagate 

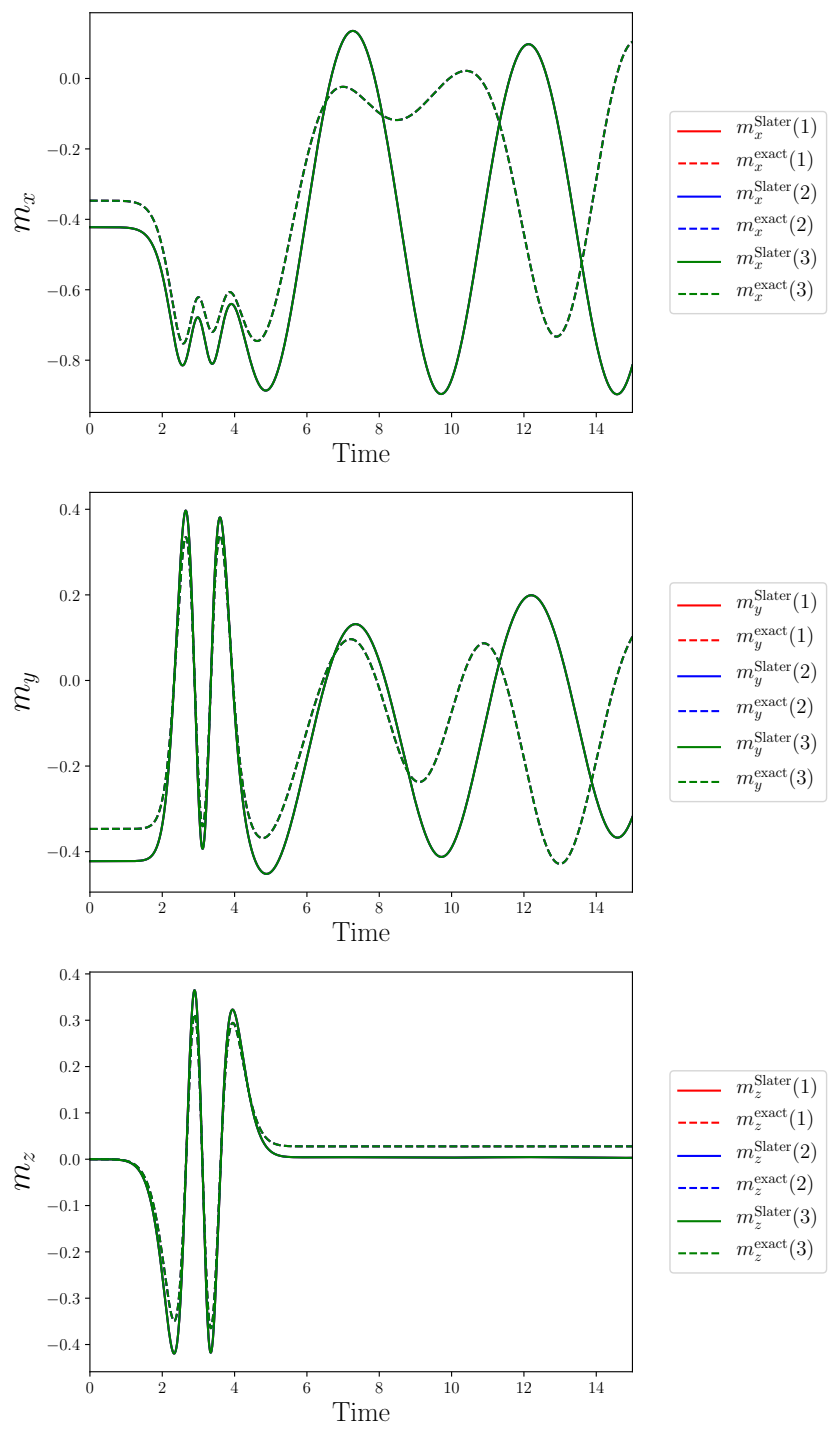

Figure 5.5: Magnetization differences between the exact magnetizations (dashed lines) and Kohn-Sham magnetizations (filled lines) in the $x$-, $y$-, and $z$-directions for the first (red), second (blue), and third (green) lattice sites with $C=0.4$ and $T=0.2309$. 
in time, the system behaves collectively, staying in a collinear state throughout the time propagation. This could be due to the fact that the magnetic field applies the same force on each site equally, so no spins precess differently compared to their neighboring sites. Because of this, the spins evolve the same, with the SOC allowing for the continuation of the precession after the pulse subsides. Still, we see that the SOC doesn't appear to cause any spin mixing effects, where neighboring sites might influence the precession of another, causing electrons to influence the spin precession of their neighbors.

Selecting a point just above the phase boundary, at $C=0.4$ and $t=0.231$, we immediately see that there is a vastly different spin orientation. Quickly, we see that we are in a noncollinear spin configuration, where each lattice site is pointed out from the center of the triangle. Applying our magnetic pulse to this spin configuration, the behavior of the system is very different from before. Instead of a collective evolution, we see in Fig. 5.6 that now all three sites evolving independently. Part of the effect could simply be due to the fact that the magnetic field is hitting each site differently this time; more important, however, seems to be the spin configuration causing the SOC term (which, recall, acts as a complex nearest-neighbor hopping) to allow for spin effects that alter the precession of neighboring lattice electrons.

Regardless of the spin state in which we choose to prepare our system, we clearly see that, at least at the start of the time propagation and through the magnetic pulse, the KS Slater solution performs admirably well. In the transient phase after the magnetic pulse, there is a clear divergence away from the exact solutions in both situations though. Broadly speaking, despite quickly diverging from the exact solution through the transient period, it appears that the phase of the precession well after 

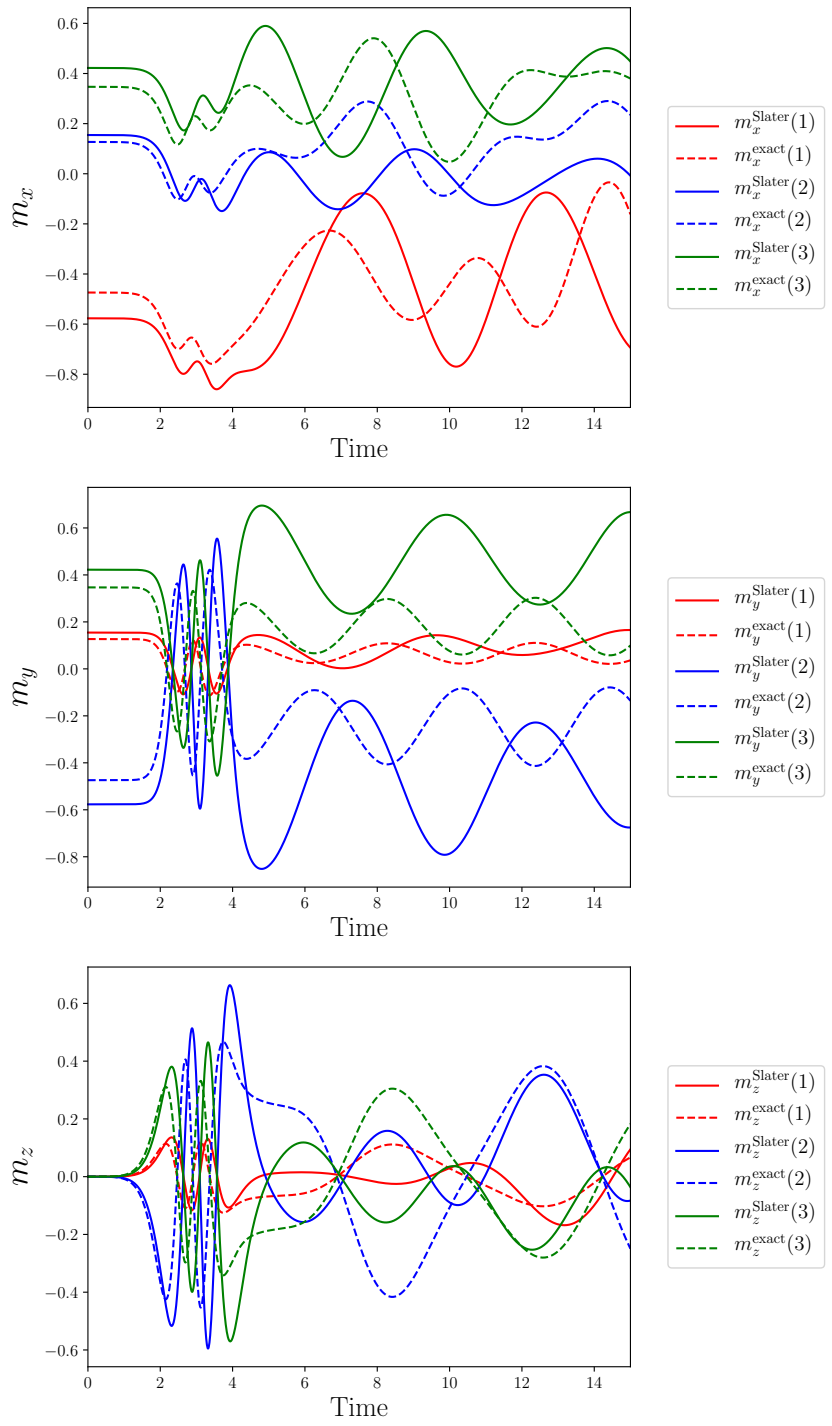

Figure 5.6: Magnetization differences between the exact magnetizations (dashed lines) and Kohn-Sham magnetizations (filled lines) in the $x$-, $y$-, and $z$-directions for the first (red), second (blue), and third (green) lattice sites with $C=0.4$ and $T=0.231$. 
the pulse is similar, perhaps pointing to good agreement between the KS and exact solutions despite the poor performance through the transient phase. In general, the KS solution captures the oscillations associated with the precessing magnetic moments rather well, at least qualitatively. A spectral analysis, to be performed in future work, might be able to provide more quantitative details.

\subsection{Time propagation of the tetramer}

In Chapter 4, we focused on the ground state of the tetramer system and noted the different regimes that were present where we had low and high interaction strength. In the high interaction strength regime, we saw that we had electronic localization and xc torques that were larger than the external torque because of the magnetization aligning with the magnetic fields. On the opposite end, the external torques dominated the xc torques in the low interaction strength regime. Between both regimes, however, was a sizeable crossover region where the external and xc torques were on the same order of magnitude as one another.

We postulated that this mismatch and these two regimes could lead to large discrepancies between the exact and KS solutions, meaning that correctly accounting for the torques would be very important. By using the same method as we used in the trimer, we can begin to explore whether our hypothesis will bear fruit. In Fig. 5.7, we can clearly see that the low interaction strength regime has little to no change between the full $\mathbf{B}_{\mathrm{xc}}$ and $\mathbf{B}_{\mathrm{xc}, \|}$ (where the transverse component of the xc magnetic field and thus the torques have been projected out). This would follow our intuition as the xc torques were shown to be small in this regime. 

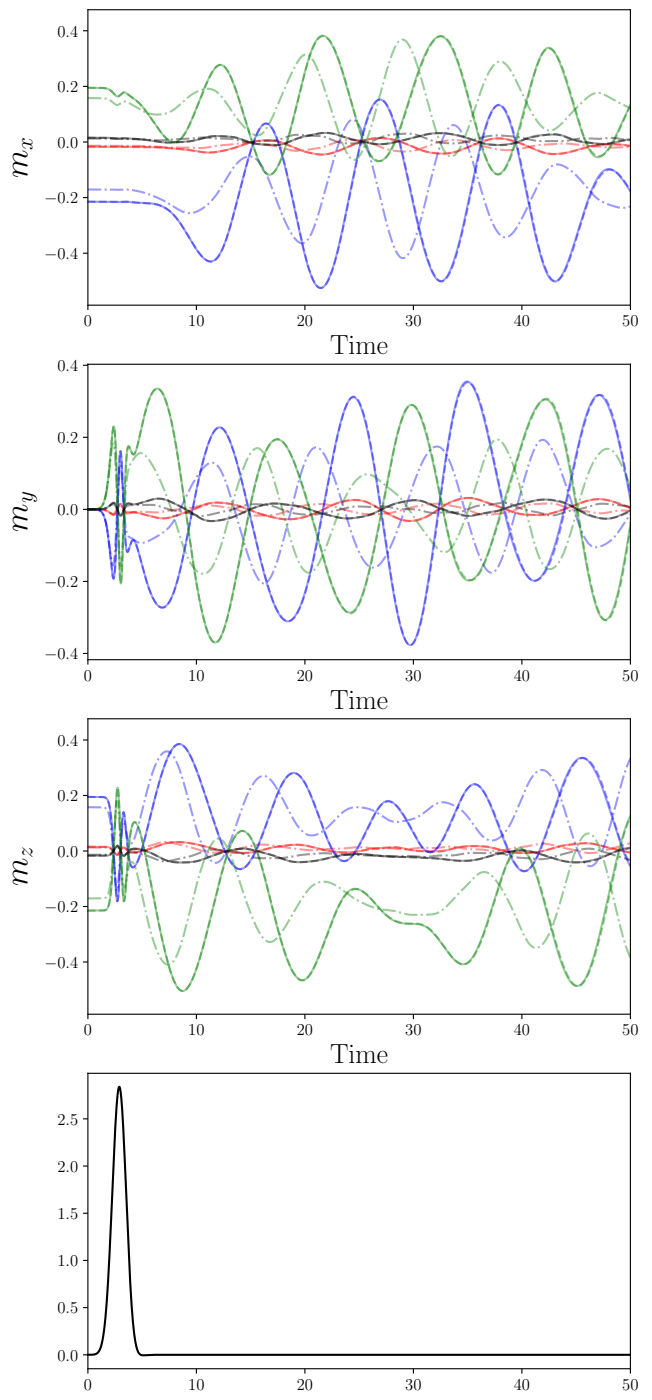

Figure 5.7: Magnetization differences between the exact magnetizations (dash-dotted lines) and Kohn-Sham magnetizations ( $\mathbf{B}_{\mathrm{xc}, \|}$ :filled lines, $\mathbf{B}_{\mathrm{xc}, \text { full }}$ : dashed lines) in the $x-, y$-, and $z$-directions for the first (red), second (blue), third (green), and fourth (grey) lattice sites with $U_{0}=1.0$. 

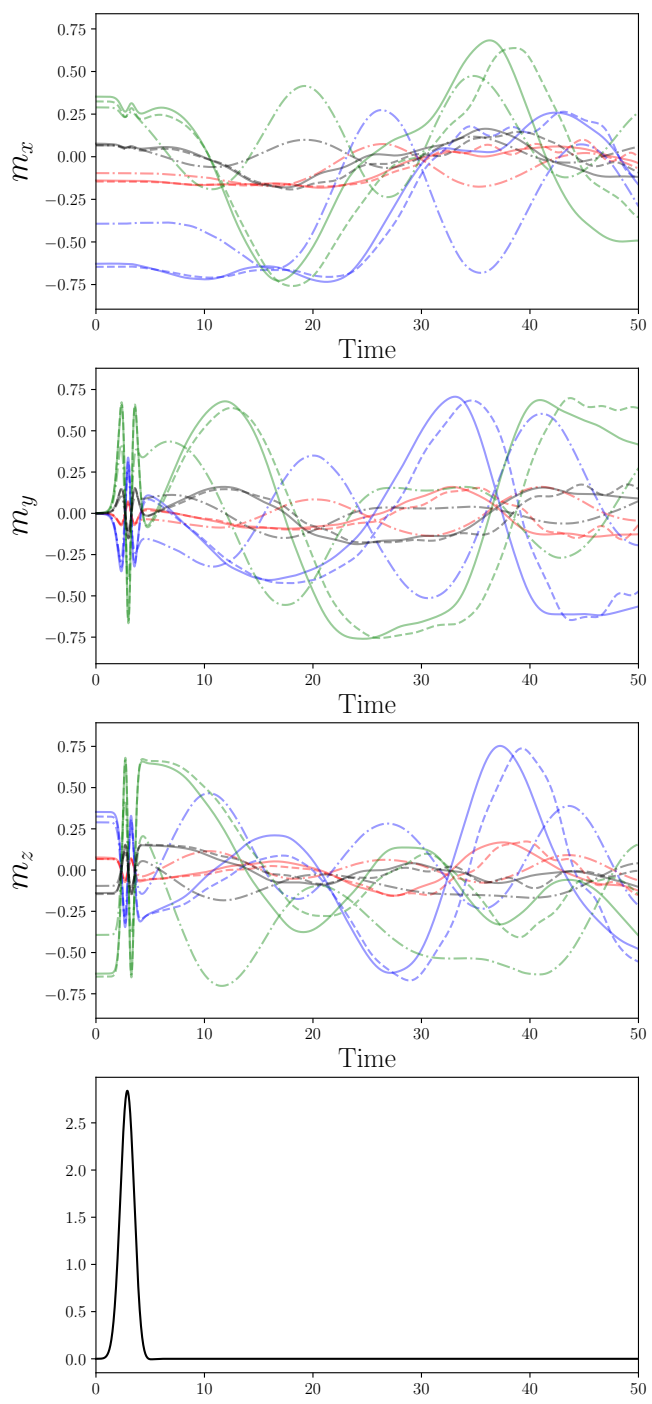

Figure 5.8: Magnetization differences between the exact magnetizations (dash-dotted lines) and Kohn-Sham magnetizations ( $\mathbf{B}_{\mathrm{xc}, \|}$ :filled lines, $\mathbf{B}_{\mathrm{xc}, \text { full }}$ : dashed lines) in the $x$-, $y$-, and $z$-directions for the first (red), second (blue), third (green), and fourth (grey) lattice sites with $U_{0}=3.0$. 
Such is not the case in Fig. 5.8. Interestingly, as we enter the crossover regime, we begin to see that by removing the torques, we cause a clear change in the dynamics of the magnetizations. Throughout the magnetic pulse, the solutions, both full and with the longitudinal xc field only, stay fairly close together and do well to recreate the exact solution. However, once the pulse is completed, the solutions begin to separate, becoming very different near $t=15$ and showing no signs that the gap between the two solutions will close. Clearly, this would indicate that it is necessary to account for these torques as they can give rise to very different magnetization dynamics.

Finally, we go all the way to the high interaction strength regime, and examine what we showed in Chapter 4 to be where the xc torques dominated over the external torques. Here, as shown in Fig. 5.9, it is interesting that the xc torque doesn't seem to have an accumulative behavior when the transverse xc magnetic field is removed. We still see large differences in the steady state solution by the difference in the starting magnetization values, but as the magnetization time evolves, there are no notable difference in how large the disparity is between the full and longitudinal solutions. Despite this, there is a clear difference between the full and longitudinal solutions throughout the time evolution of the magnetizations, demonstrating that the torques play a role in the steady state solution, but it isn't clear that the behavior worsens over time.

\subsection{Summary and conclusions}

As mentioned previously, understanding xc torque effects on the dynamics and evolution of real systems is necessary for properly characterizing a systems behavior. By 

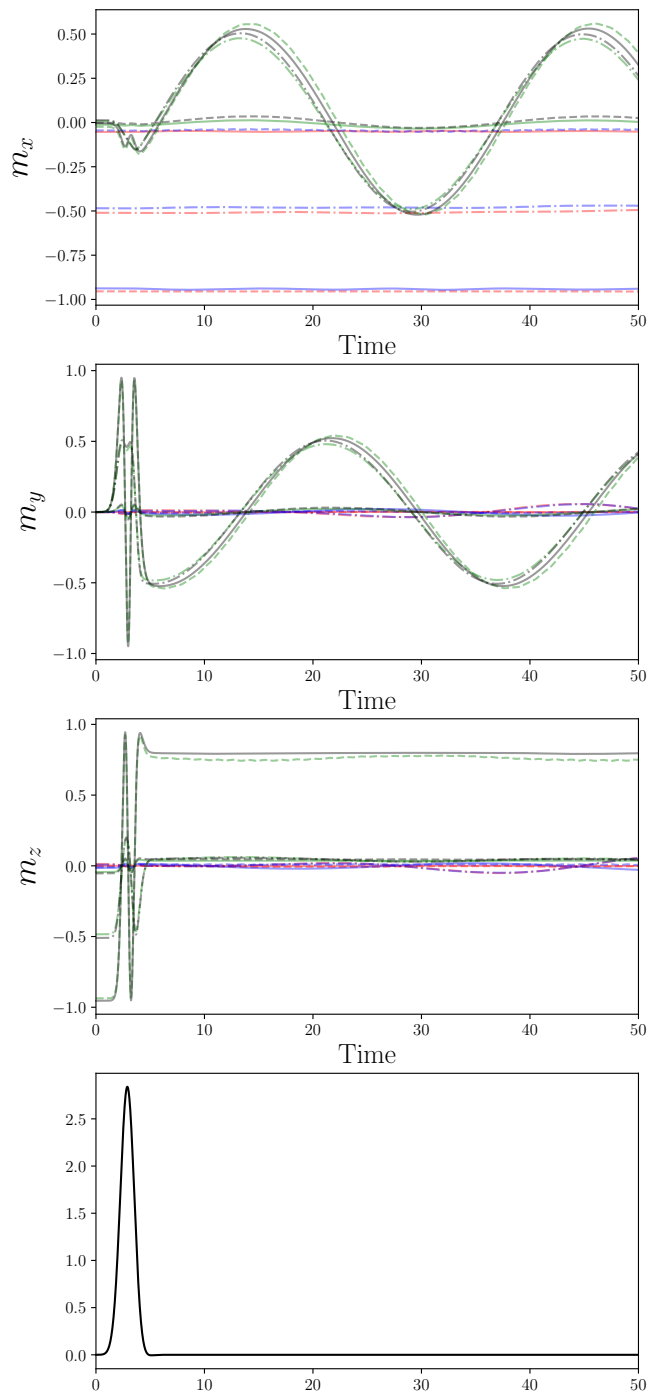

Figure 5.9: Magnetization differences between the exact magnetizations (dash-dotted lines) and Kohn-Sham magnetizations ( $\mathbf{B}_{\mathrm{xc}, \|}$ :filled lines, $\mathbf{B}_{\mathrm{xc}, \text { full }}$ : dashed lines) in the $x$-, $y$-, and $z$-directions for the first (red), second (blue), third (green), and fourth (grey) lattice sites with $U_{0}=9.0$. 
exciting a trimer system with SOC, we were able to correctly capture the local, effectively changing magnetic field, demonstrated by the magnetic behavior seen in Fig. 5.4. With the inclusion of the SOC term, we effectively included a local magnetic field that continued to fluctuate after the termination of the magnetic field that was incident on the system.

By approaching a phase boundary between two degenerate spin states, we see that we get extremely different behavior depending on the state that was prepared. Additionally, the states appear to be very robust, even when exposed to a magnetic field pulse that serves as a quick and decisive kick to the system. We see that there doesn't appear to be a jump from one state to another, despite being near the phase boundary. That said, the SOC term in the Kohn-Sham solution shows extremely interesting physical properties with collective precession and independent precession with very small fluctuations in parameter space.

When we revisited the tetramer, we saw that despite the xc torques being dominant over some interaction strength regimes, it doesn't appear that these differences increase over time for all regimes. We saw what would be expected in the low interaction strength regime, where there was little difference between the Kohn-Sham approximations where the full xc fields were included or were projected out. Interestingly, at high interaction strengths, the large xc torques don't seem to change or cause large differences in how the tetramer magnetizations evolve. In some sense, this could be that KS approximations already poorly recreate exact solutions in strongly correlated regimes, so there would be no reason to assume projecting the torques out would cause a large change. Finally, in the crossover regime, there are clear differences in the evolutions of the longitudinal and full xc field calculations. After the 
magnetic field terminates, it is clear that the evolution of the magnetizations start to change, warranting further investigation to determine why this crossover regime experiences such interesting dynamic changes.

The numerical calculations presented in this Chapter are clearly not comprehensive, but merely exploratory. In the ground-state calculations we had seen that xc torques have little overall effects on the energetics of the system. What we wanted to see was whether there are any indications that, in the dynamical regime, small differences can dramatically accumulate over time. The results shown in this Chapter indicate that there are regimes where this can happen, but in other regimes the differences remain small.

Needless to say, our calculations used only the Slater approximation, which completely ignores any correlation effects. Thus, a full comparison with exact results can only be performed at a qualitative level. Nevertheless, even the simple Slater approximation can be useful. Further tests, including realistic first-principles calculations, are certainly needed to arrive at a more complete picture.

Another interesting aspect of the time-dependent calculations discussed here has to do with recent studies on ultrafast switching of ferromagnetic thin films [88]. There, it was found that a short laser pulse can lead to a fast, permanent switching of the magnetization of the ferromagnet. The authors of Ref. [88] argue that the main mechanism of this ultrafast switching is due to SOC. The Hubbard trimer calculations we have presented here are not quite comparable to this scenario, but even then we have seen that the presence of SOC can profoundly alter the response of magnetic systems at an ultrafast level. Again, this calls for further studies in the future. 


\section{Chapter 6}

\section{Summary and future direction}

We've laid bare how model systems can be used to examine the methods and ideas used to explore and push the boundaries of physics. While simplified, a properly chosen model can explore and target different model properties that might be obscured by the complexities present in real materials. By boiling down the system to its most basic components, we have shown that researchers can examine and explore the basic underpinnings of the theories that support the wide and rich research area of condensed matter physics.

\subsection{Summary}

In this dissertation we explored model systems, specifically lattice systems and onedimensional potential wells decorated with additional potential differences. Beginning from the Casida formalism, we applied a unitary transformation to explore how the particle-hole map, which is derived from the density response, can be used to examine 
functional units within excitations. While in principle any unitary transformation could be used, we created an optimization technique that can be generalized into extended systems.

Taking a step further into the Kohn-Sham formalism, we applied spin-dependent density-functional theory onto a Hubbard tetramer where we were able to exactly solve the full Schrödinger equation analytically. We also developed a conjugate gradient optimization scheme that allowed us to directly reconstruct the exact exchangecorrelation (xc) potential and magnetic field, and provide interesting insight into xc torque regimes and their interaction strength dependencies. Building up from the ground-state tetramer solutions, we performed an exploratory analysis on the spin dynamics of the Hubbard tetramer when under the influence of a time-dependent magnetic field. The torques showed extremely interesting time-dependent behavior, warranting further study. Finally, we also examined the Hubbard trimer, exploring how spin-orbit coupling allowed us to explore degenerate spin-states and examine the dynamics of a small quantum system near a phase boundary.

In Chapter 3, we introduced the particle-hole map (PHM) which is a visualization tool derived from the density response function. When compared to a similar object, the transition density matrix (TDM), we saw that the PHM is not invariant under a unitary transformation of the canonical orbital wavefunctions. While one could recover the canonical PHM, we defined a new quantity that was analogous to the PHM which used only the localized wavefunctions. Using our newly defined localized particle-hole map (LPHM), we optimized our orbital transformation according to the Foster-Boys method, which is analogous to Wannier orbitals in extended systems, separating our orbital density so that there is as little overlap as possible. Applying 
our methods to a simple one-dimensional system, where we could control all inputs, we saw that even in simplistic cases, we were able to see interesting and promising behavior. Through the LPHM, we saw that some potential systems could be examined according to the different potential wells present, and that the LPHM shows additional layers of mediation in the charge excitations that the PHM simply did not show.

After optimizing orbitals spatially, in Chapter 4 we changed gears and utilized the analytic solvability of a Hubbard system to examine and exactly solve for the exchange-correlation term in various functional approximations. By beginning from the two site Hubbard model, we generalized the system to exactly solve the Schrödinger equation for an $n$-site system. As we discussed, we chose the four site Hubbard model with nearest-neighbor interactions because it was the simplest system that allowed for exchange-correlation spin torques. Also, since we could analytically solve the fully interacting Schrödinger equation for the tetramer, we were able to explore the differences of various functional approximations within the Kohn-Sham formalism. By inverting the Kohn-Sham equations, we could apply computational optimization methods to solve for the density of our system to within machine precision of the exact analytic solution. We chose to use the conjugate gradient method and showed that we were consistently able to converge our Kohn-Sham density to within $10^{-} 7$ of the exact density given by the Schrödinger equation. Because of this, we could directly compare the exact potentials to their Kohn-Sham counterparts and recover the xc fields that were needed to recreate our exact density. Interestingly, exchangeonly methods are at least adequate in the weakly correlated electron regime, where many systems of interest lie (namely those with relatively light nuclei). Additionally, we saw that there is in fact a sort of saturation moving towards the highly correlated 
regime where electron density localizes and the electron spins align with the applied external magnetic field. This means that the xc magnetic fields become at least on the order of the external magnetic fields, showing that these xc fields can have large effects in highly correlated regimes and must be accounted for in those calculations.

Continuing to examine an extension of the Hubbard model, in Chapter 5, we saw that including spin orbit coupling (SOC) has a large effect on the real-time electron dynamics in a Kohn-Sham system where the xc fields are allowed to lie non-parallel to the magnetization. Once again, using the Hubbard model allowed us to solve the Schrödinger equation via exact diagonalization of the system, where we could then compare the exact solution and time evolution to the KS evolution over time. We began by exploring the spin configuration phase diagram to examine the phase space dependence on the SOC and the hopping constant (kinetic energy). By exploring the phase space, we were able to approach the phase boundary and prepare our initial state in collinear and noncollinear spin states, exploring the spin dynamics of the trimer under an applied magnetic field. Even exchange-only functionals like the Slater approximation do qualitatively well at capturing the spin dynamics of the trimer, with well-defined spin dynamic regimes where the spins precessed in unison or propagated independently.

Finally, we explored the time evolution of the xc torque effects in the Hubbard tetramer system. We saw that there are regimes where we see expected behavior, such as the low interaction limit, where the torques play little-to-no role in the spin dynamics. However, in the crossover interaction strength regime, where the xc torques are at least on the order of the spin torques due to the applied magnetic field, there appears to be a qualitative accumulation effect, where the torques seem to cause 
an increasing change to the spin dynamics of the system. In the high interaction strength regime, we also saw that despite a clear difference between torque included and projected out solutions, the difference appears to stay constant throughout the time evolution.

\subsection{Future direction}

The research that has been accomplished exposes and allows for future exploration into interesting physics that has only just begun to be looked at in depth. From spin dynamics and their applications in spintronics to charge transport and transfer in semiconductors and photovoltaics, there is a rich exploration landscape that can be developed from atop this thesis. We will briefly exlore a few different possible directions for that research in the following sections.

\subsubsection{Charge and spin transfer in finite systems}

In Chapter 3, we explored a simple one-dimensional potential well and demonstrated a visualization tool that could be used to probe possible behavior and characteristics of organic photovoltaics. Intramolecular charge transfer is one such area where there could be a considerable use for the LPHM to shed light on the characteristics of the excitation. One such molecule that can be used an example is the benzothiadiazole (BT) molecule terminated with thiophene units that act as charge donors. As can be seen in Fig. 6.1, the canonical orbitals are extended over the entire molecule without particular concentration on any units. While the Foster-Boys orbital localization method is scalable to three dimensions, and could be used to calculate localized or- 


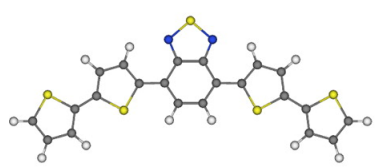

(a)

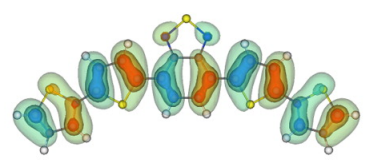

(d)

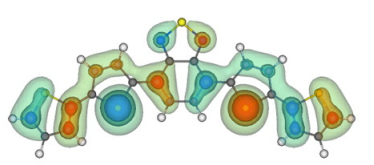

(b)

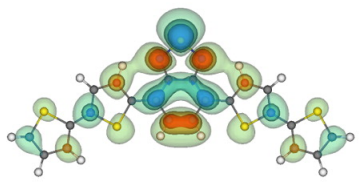

(e)

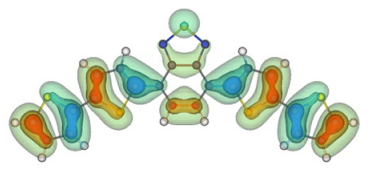

(c)

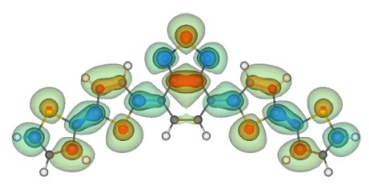

(f)

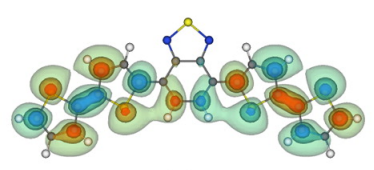

(g)

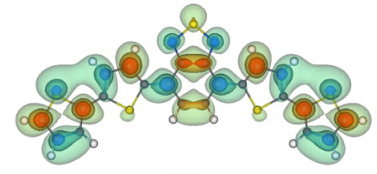

(h)

Figure 6.1: (a) Structure of thiophene terminated benzothiadiazole (BT) and the canonical orbitals of the (b) HOMO-2, (c) HOMO-1, (d) HOMO, (e) LUMO, (f) $\mathrm{LUMO}+1$, (g) LUMO+2, and (h) LUMO+3 orbitals. Adapted from [63].

bitals by minimizing the orbital overlap, any proper choice of localization method could be chosen to transform the canonical orbitals. Since the BT unit in Fig. 6.1 is believed to play a large role in mediating the charge transfer between the thiophene units, we could expect to localize the $\pi$-type orbitals that mediate the electron charge transfer in these organic molecules. By doing so, one could potentially gain a finer grained insight into the role each molecular unit plays during a charge transfer excitation in general, since many organic photovoltaics are made of donor-acceptor systems where the roles are played by different molecules or functional units [130-132].

More generally, we could expect to use localized orbitals in any situation where we would expect electrons to have large orbital ranges that extend across "large" distances within the system. In addition to the canonical electronic orbitals, one could use the so-called naturally localized orbitals and use the same methods outlined in 3. Finally, simply expanding the PHM into spin-dependent quantities could provide 
some insight into spin dynamics in molecules and clusters. By using noncollinear spin states, where we can depict the wavefunctions as spinors, we could potentially investigate spin flip dynamics as well as spin-dependent excitations within molecular systems. With organic materials becoming popular in the fabrication of many electronic systems, understanding and demonstrating the spin dynamics of molecular magnets could expose the underlying mechanisms that are at work within these molecules. Once the mechanisms are more fully understood, we could potentially find more candidates that could be used in one of the many applications of magnetics, namely quantum computers and data storage [133,134].

Beyond clusters and finite systems, the LPHM can be easily generalized to extended systems. As we mentioned in our work exploring 1D systems, the Foster-Boys method of orbital localization is analogous to the Wannier states in an extended system. Because of this, the LPHM could potentially be used as a tool with Wannier wavefunctions to see if there is localized charge transport and explore excitonic processes in the basis of a new localized wavefunction. Additionally, the LPHM could be generalized to give a dynamical picture of excitation processes in real time.

\subsubsection{Spin dynamics in noncollinear, spin-frustrated materi- als}

As was mentioned in Chapters 4 and 5 , the effects of localized xc torques on time dependent calculations for real systems has hardly been touched by researchers. Generally, researchers tend to use the localized spin-density approximation (LSDA) to trivially satisfy the requirement that there be no net xc torque felt by our electronic density. From what we found in Chapter 4, this should be a good approximation 
when we are in a regime that is heavily dominated by the external magnetic field. Unfortunately, for many systems, we are not in that large external magnetic field either throughout the duration of our electron dynamics or at all. Even so, in weakly correlated materials, where electronic interactions can be better approximated by our noninteracting model, we should still be able to manage for many situations with the LSDA method because of the tiny size of magnetization differences due to the xc fields. When we approach a degenerate state, however, the xc fields play a much larger role in maintaining equilibrium and thus the effects created by those xc fields cannot be satisfied by the LSDA. What this implies is simply that when we have a system that has possible degeneracies, we must take increased care to make sure our xc torques are monitored and calculated as accurately as possible.

There are groups of systems where these spin degeneracies are readily apparent and they can go by many different names: spin ice, spin glass, frustrated molecules. In these materials, the spins of the materials are aligned or anti-aligned in such a way that we cannot satisfy the spin orientation of each lattice site/atom simultaneously. In other words, there will always be a single site where there is an ambiguous orientation of the spin moment of the electron. The electron(s) are then in a near, or fully, degenerate state, where there are competing magnetic forces trying to align the electronic spin(s) into competing configurations. Knowing how these spins evolve over time is crucial to understanding where our approximations break down and learning the systematic ways in which our approximations might fail.

Such non-collinear, spin-frustrated materials [135] can be studied using 2D model Hamiltonians such as triangular or Kagome lattices [128], or through first-principles approaches to materials such as Cr monolayers [103], pyrochlores [136], or molecu- 
lar magnets [137]. Since orbital-based exchange-correlation functionals are not constructed based on the homogeneous electron gas as reference system, they can be used for model and real systems alike.

\subsubsection{Continued optimization of functionals and inversion methods}

While the previous sections primarily focused on new areas of research, here we will focus on the ability to continue to incrementally improve the methods used throughout this thesis work. Chapters 3 and 4 utilized optimization routines to calculate target quantities with a known distribution. While the model systems involved were simple, it allowed for the exploration of numerical routines meant to optimize these many dimensional spaces. In our favor, the Foster-Boys method has been implemented for programs that are commonly used throughout chemistry and physics $[138,139]$. Unfortunately, our inversion technique of the KS equations is not so performant in real situations. In the case of our inversion routine, the limitation is the number of points for which our KS wavefunctions must be optimized.

We implemented the conjugate gradient method to converge our model system, but there are many more potential near degeneracies in real systems, making the solution space more complex and the odds of finding a global minimum (maximum) more difficult. Because of the added complexity of the system, its likely that the conjugate gradient method would be computationally poor for larger solution space systems where there could be more local minima that in our model problem. There are proposed methods already in use for more realistic systems $[114,140]$, but the difficulty is in removing the additive constant of our scalar potential. The additive 
constant in a larger and more complex solution space gives rise to an infinite number of solutions, making it difficult to converge towards a solution without careful selection of a system that should behave well for the inversion. One possible method to invert the KS equations could be to do an ensemble method where each individual starting point moves towards a local minima. By casting a wide net to find numerous possible solutions at one time, it could, feasibly, be possible to approach a pseudo-global minimum (maximum) that solves for the nondegenerate ground state of our system. Machine learning would be another possibility worth exploring. Regardless of the method, a limiting factor would still be the grid size of the real space system we are solving for, as we would need an $N \times N$ size matrix (without spin dependence) stored in memory to calculate the gradient steps of our wavefunction phase space.

One primary focus in much of this thesis has been on functional selection and functionals that are performant regardless of the system. While not there yet, the Slater functional (or the Exact Exchange Potential (EXX) in DFT formalism) has promising features that might allow it to take hold in many computational calculations. Even though the Slater approximation and EXX are not as simple as LSDA, the potential only relies on local quantities, so computationally it is not terribly difficult to implement. The performance drawbacks with the EXX and other non-trivial xc torque satisfying functionals are borne entirely out of needing to store the entire wavefunction in memory for all lattice sites. Our approximations need these values in memory because they rely explicitly on the wavefunction (and sometimes even its gradient) at all points in space, whereas LSDA does not. For noncollinear systems then, we could more accurately take into account the xc fields that are affecting our system and more accurately approximate how those fields evolve over time. 


\subsubsection{Tetramer and Trimer Conclusion}

In Chapter 5, we discussed the additional analysis done on the tetramer and trimer systems. As mentioned, much of the calculations and analysis were merely exploratory in nature. We saw that even just scratching the surfaces of the Hubbard systems, there is a rich and exciting area of physics research to be explored. Beyond qualitative analysis of the time dependent magnetizations in the exact and $\mathrm{KS}$ solutions, we can further perform spectral analysis on the magnetizations to quantitatively state the similarities between the solutions. We also only explored exchange-only timedependent effects, leaving correlation effects for later study. By including correlation, via the STLS method or even a Møller-Plesset perturbation, we would also be able to explore additional dynamic effects that aren't accounted for by simple exchange-only methods like Slater. 


\section{Bibliography}

[1] E. Schrödinger. An undulatory theory of the mechanics of atoms and molecules. Phys. Rev., 28:1049-1070, 1926.

[2] M. Born, W. Heisenberg, and P. Jordan. Zur Quantenmechanik. II. Zeitschrift für Physik, 35:557-615, 1926.

[3] P. A. M. Dirac. The fundamental equations of quantum mechanics. Proceedings of the Royal Society A, 109(752):642-653, 1925.

[4] M. Born and R. Oppenheimer. Zur Quantentheorie der Molekeln. Annalen der Physik, 389(20):457-484, 1927.

[5] M. Born and V. Fock. Beweis des Adiabatensatzes. Zeitschrift für Physik, 51(3):165-180, 1928.

[6] S. Moriyama, T. Fuse, M. Suzuki, Y. Aoyagi, and K. Ishibashi. Importance of electron-electron interactions and Zeeman splitting in single-wall carbon nanotube quantum dots. Physica E: Low-dimensional Systems and Nanostructures, 26(1):473-476, 2005 . 
[7] G. Kotliar, S. Y. Savrasov, K. Haule, V. S. Oudovenko, O. Parcollet, and C. A. Marianetti. Electronic structure calculations with dynamical mean-field theory. Rev. Mod. Phys., 78:865-951, 2006.

[8] D. Vollhardt. Dynamical mean-field theory for correlated electrons. Annalen der Physik, 524(1):1-19, 2012.

[9] P. Hohenberg and W. Kohn. Inhomogeneous electron gas. Phys. Rev., 136:B864, 1964.

[10] Mel Levy. Universal variational functionals of electron densities, first-order density matrices, and natural spin-orbitals and solution of the v-representability problem. Proceedings of the National Academy of Sciences, 76(12):6062-6065, 1979.

[11] Elliott H. Lieb. Density functionals for Coulomb systems. Int. J. of Quant. Chem., 24(3):243-277, 1983.

[12] J. C. Slater. The theory of complex spectra. Phys. Rev., 34:1293-1322, 1929.

[13] W. Kohn and L. J. Sham. Self-consistent equations including exchange and correlation effects. Phys. Rev., 140:A1133, 1965.

[14] Kieron Burke. Perspective on density functional theory. J. Chem. Phys., 136(15):150901, 2012.

[15] Andrea Dal Corso, Stefano Baroni, and Raffaele Resta. Density-functional theory of the dielectric constant: Gradient-corrected calculation for silicon. Phys. Rev. B, 49:5323-5328, 1994. 
[16] Young-Moo Byun, Jiuyu Sun, and Carsten A Ullrich. Time-dependent density-functional theory for periodic solids: assessment of excitonic exchange-correlation kernels. Electronic Structure, 2(2):023002, 2020.

[17] Vy Tran, Ryan Soklaski, Yufeng Liang, and Li Yang. Layer-controlled band gap and anisotropic excitons in few-layer black phosphorus. Phys. Rev. B, 89:235319, 2014.

[18] John P. Perdew, Kieron Burke, and Matthias Ernzerhof. Generalized gradient approximation made simple. Phys. Rev. Lett., 77:3865-3868, 1996.

[19] A. D. Becke. Density-functional exchange-energy approximation with correct asymptotic behavior. Phys. Rev. A, 38:3098-3100, 1988.

[20] Chengteh Lee, Weitao Yang, and Robert G. Parr. Development of the ColleSalvetti correlation-energy formula into a functional of the electron density. Phys. Rev. B, 37:785-789, 1988.

[21] S. H. Vosko, L. Wilk, and M. Nusair. Accurate spin-dependent electron liquid correlation energies for local spin density calculations: a critical analysis. Canadian J. Phys., 58(8):1200-1211, 1980.

[22] E. Runge and E. K. U. Gross. Density-functional theory for time-dependent systems. Phys. Rev. Lett., 52:997, 1984.

[23] E.K.U. Gross and W. Kohn. Time-dependent density-functional theory. In PerOlov Löwdin, editor, Density Functional Theory of Many-Fermion Systems, volume 21 of Advances in Quantum Chemistry, pages 255 - 291. Academic Press, 1990. 
[24] Roi Baer and Recca Gould. Method for ab initio nonlinear electron-density evolution. J. Chem. Phys., 114:3385, 2001.

[25] Rongqing Chen and Hua Guo. The Chebyshev propagator for quantum systems. Computer Physics Communications, 119(1):19 - 31, 1999.

[26] Cleve Moler and Charles Van Loan. Nineteen dubious ways to compute the exponential of a matrix, twenty-five years later. SIAM Review, 45(1):3-49, 2003.

[27] Marlis Hochbruck and Christian Lubich. On Magnus integrators for timedependent Schrödinger equations. SIAM J. Numerical Analysis, 41:945-963, 2003.

[28] Nicolas Tancogne-Dejean, Michael A. Sentef, and Angel Rubio. Ultrafast modification of Hubbard $U$ in a strongly correlated material: Ab initio high-harmonic generation in NiO. Phys. Rev. Lett., 121:097402, 2018.

[29] Nicolas Tancogne-Dejean, Oliver D. Mücke, Franz X. Kärtner, and Angel Rubio. Impact of the electronic band structure in high-harmonic generation spectra of solids. Phys. Rev. Lett., 118:087403, 2017.

[30] Nicolas Tancogne-Dejean and Angel Rubio. Atomic-like high-harmonic generation from two-dimensional materials. Science Advances, 4(2):eaao5207, 2018.

[31] X. Andrade, D. A. Strubbe, U. De Giovannini, A. H. Larsen, M. J. T. Oliveiraa, J. Alberdi-Rodriguez, A. Varas, I. Theophilou, N. Helbig, M. Verstraete, L. Stella, F. Nogueira, A. Aspuru-Guzik, A. Castro, M. A. L. Marques, and A. Rubio. Real-space grids and the Octopus code as tools for the development 
of new simulation approaches for electronic systems. Phys. Chem. Chem. Phys., $17: 31371,2015$.

[32] F. Gygi. Architecture of Qbox: A scalable first-principles molecular dynamics code. IBM Journal of Research and Development, 52(1.2):137-144, 2008.

[33] André Schleife, Yosuke Kanai, and Alfredo A. Correa. Accurate atomistic firstprinciples calculations of electronic stopping. Phys. Rev. B, 91:014306, 2015.

[34] M. A. L. Marques, N. T. Maitra, F. M. S. Nogueira, E. K. U. Gross, and A. Rubio, editors. Fundamentals of Time-dependent density-functional theory. Springer, 2012.

[35] Mônica A. Cotta. Quantum dots and their applications: What lies ahead? ACS Applied Nano Materials, 3(6):4920-4924, 2020.

[36] N.W. Ashcroft and N.D. Mermin. Solid State Physics. Saunders College, Philadelphia, 1976.

[37] R. C. Ashoori. Electrons in artificial atoms. Nature, 379:413-419, 1996.

[38] M Milun, P Pervan, and D P Woodruff. Quantum well structures in thin metal films: simple model physics in reality? Reports on Progress in Physics, 65(2):99-141, 2002.

[39] Marc A. Kastner. Artificial atoms. Physics Today, 46(1):24, 1993.

[40] Zh. I. Alferov. The history and future of semiconductor heterostructures. Semiconductors, 32(1), 1998. 
[41] C. A. Ullrich and G. Vignale. Collective charge-density excitations of noncircular quantum dots in a magnetic field. Phys. Rev. B, 61:2729-2736, 2000.

[42] Mervyn Roy. The tight binding method. http://www.physics.rutgers.edu/ eandrei/chengdu/reading/tight-binding.pdf, 2015.

[43] S Forti and U Starke. Epitaxial graphene on SiC: from carrier density engineering to quasi-free standing graphene by atomic intercalation. Journal of Physics D: Applied Physics, 47(9):094013, 2014.

[44] Sergej Konschuh, Martin Gmitra, and Jaroslav Fabian. Tight-binding theory of the spin-orbit coupling in graphene. Phys. Rev. B, 82:245412, 2010.

[45] Aaron Bostwick, Taisuke Ohta, Thomas Seyller, Karsten Horn, and Eli Rotenberg. Quasiparticle dynamics in graphene. Nature Physics, 3:36-40, 2007.

[46] The Hubbard model at half a century. Nature Physics, 9(523), 2013.

[47] Edward A. Pluhar and Carsten A. Ullrich. Visualizing electronic excitations with the particle-hole map: orbital localization and metric space analysis. The European Physical Journal B, 91(137), 2018.

[48] C. Deibel, T. Strobel, and V. Dyakonov. Role of the charge transfer state in organic donor-acceptor solar cells. Adv. Mater., 22:4097-4111, 2010.

[49] E. K. U. Gross, J. F. Dobson, and M. Petersilka. Density functional theory of time-dependent phenomena. In R. F. Nalewajski, editor, Density-Functional Theory II, volume 181 of Topics in Current Chemistry, pages 81-172. Springer, Berlin, 1996. 
[50] C. A. Ullrich. Time-dependent density-functional theory: Concepts and applications. Oxford University Press, 2012.

[51] T. Burnus, M. A. L. Marques, and E. K. U. Gross. Time-dependent electron localization function. Phys. Rev. A, 71:010501-1-4, 2005.

[52] S. Tretiak and S. Mukamel. Density matrix analysis and simulation of electronic excitations in conjugated and aggregated molecules. Chem. Rev., 102:3171, 2002.

[53] Filipp Furche. Molecular tests of the random phase approximation to the exchange-correlation energy functional. Phys. Rev. B, 64:195120-1-8, 2001.

[54] Felix Plasser, Michael Wormit, and Andreas Dreuw. New tools for the systematic analysis and visualization of electronic excitations. I. Formalism. J. Chem. Phys., 141:024106, 2014.

[55] Thibaud Etienne. Transition matrices and orbitals from reduced density matrix theory. J. Chem. Phys., 142:244103, 2015.

[56] Kevin M. Coakley and Michael D. McGehee. Conjugated polymer photovoltaic cells. Chem. Mater., 16(23):4533-4542, 2004.

[57] Jerzy Cioslowski, editor. Many-electron densities and reduced density matrices. Kluwer Academic / Plenum Publishers, New York, 2000.

[58] Per-Olov Löwdin. Quantum theory of many-particle systems. I. Physical interpretations by means of density matrices, natural spin-orbitals, and convergence problems in the method of configurational interaction. Phys. Rev., 97:1474$1489,1955$. 
[59] R. McWeeny. Some recent advances in density matrix theory. Rev. Mod. Phys., 32:335-369, 1960.

[60] S. Bäppler, F. Plasser, M. Wormit, and A. Dreuw. Exciton analysis of manybody wave functions: Bridging the gap between the quasiparticle and molecular orbital pictures. Phys. Rev. A, 90:052521-1-12, 2014.

[61] Richard L. Martin. Natural transition orbitals. J. Chem. Phys., 118:4775, 2003.

[62] Z. Li, W. Liu, Y. Zhang, and B. Suo. Spin-adapted open-shell time-dependent density functional theory. II. Theory and pilot application. J. Chem. Phys., 134:134101, 2011.

[63] Y. Li and C. A. Ullrich. The particle-hole map: a computational tool to visualize electronic excitations. J. Chem. Theory Comput., 11:5838, 2015.

[64] Y. Li, D. Moghe, S. Patil, S. Guha, and C. A. Ullrich. Visualization of charge transfer excitations in donor-acceptor molecules using the particle-hole map: a case study. Mol. Phys., 114:1365, 2016.

[65] Yonghui Li and C. A. Ullrich. The particle-hole map: formal derivation and numerical implementation. J. Chem. Phys., 145:164107, 2016.

[66] J. M. Foster and S. F. Boys. Canonical configurational interaction procedure. Rev. Mod. Phys., 32:300, 1960.

[67] Clyde Edmiston and Klaus Ruedenberg. Localized atomic and molecular orbitals. Rev. Mod. Phys., 35:457, 1963. 
[68] Elvar Ö. Jónsson, Susi Lehtola, Martti Puska, and Hannes Jónsson. Theory and applications of generalized Pipek-Mezey Wannier functions. J. Chem. Theor. and Comput., 13(2):460-474, 2017.

[69] Gregory H. Wannier. The structure of electronic excitation levels in insulating crystals. Phys. Rev., 52:191-197, 1937.

[70] Gregory H. Wannier. Dynamics of band electrons in electric and magnetic fields. Rev. Mod. Phys., 34(4):645-655, 1962.

[71] Ofelia B. Oña, Diego R. Alcoba, William Tiznado, Alicia Torre, and Luis Lain. An orbital localization criterion based on the topological analysis of the electron localization function. Int. J. Quant. Chem., 113:1401, 2013.

[72] J. P. Perdew and A. Zunger. Self-interaction correction to density-functional approximations for many-electron systems. Phys. Rev. B, 23:5048-79, 1981.

[73] R. A. Heaton, J. G. Harrison, and C. C. Lin. Self-interaction correction for density-functional theory of electronic energy bands of solids. Phys. Rev. B, 28:5992-6007, 1983.

[74] M. R. Pederson, A. Ruzsinszky, and J. P. Perdew. Communication: Selfinteraction correction with unitary invariance in density functional theory. $J$. Chem. Phys., 140:121103-1-4, 2014.

[75] M. E. Casida. Time-dependent density functional response theory for molecules. In D. E. Chong, editor, Recent Advances in Density Functional Methods, volume 1 of Recent Advances in Computational Chemistry, page 155. World Scientific, Singapore, 1995. 
[76] M. Petersilka, U. J. Gossmann, and E. K. U. Gross. Excitation energies from time-dependent density-functional theory. Phys. Rev. Lett., 76:1212-5, 1996.

[77] I. D’Amico, J. P. Coe, V. V. França, and K. Capelle. Quantum mechanics in metric space: Wave functions and their densities. Phys. Rev. Lett., 106:050401, 2011.

[78] P. M. Sharp and I. D'Amico. Metric space formulation of quantum mechanical conservation laws. Phys. Rev. B, 89:115137, 2014.

[79] P. M. Sharp and I. D'Amico. Metric-space approach to potentials and its relevance to density-functional theory. Phys. Rev. A, 94:062509, 2016.

[80] Irene D'Amico, Stefano Pittalis, and Simone Marocchi. Fermionic correlations as metric distances a useful tool for materials science. Phys. Rev. Materials, 1:043801, 2017.

[81] Edward A. Pluhar and Carsten A. Ullrich. Exchange-correlation magnetic fields in spin-density-functional theory. Phys. Rev. B, 100:125135, 2019.

[82] U. von Barth and L. Hedin. A local exchange-correlation potential for the spin polarized case: I. J. Phys. C, 5:1629-42, 1972.

[83] O. Gunnarsson and B. I. Lundqvist. Exchange and correlation in atoms, molecules, and solids by the spin-density-functional formalism. Phys. Rev. B, 13:4274-98, 1976.

[84] N. I. Gidopoulos. Potential in spin-density-functional theory of noncollinear magnetism determined by the many-electron ground state. Phys. Rev. B, 75:134408-1-8, 2007. 
[85] A. J. Freeman and K. Nakamura. Modern computational magnetism: role of noncollinear magnetism in complex magnetic phenomena. Phys. Stat. Sol. (b), 241:1399, 2004.

[86] J. J. Goings, F. Egidi, and X. Li. Current development of noncollinear electronic structure theory. Int. J. Quantum Chem., 118:e25398-1-5, 2018.

[87] Egor Trushin and Andreas Görling. Spin-current density-functional theory for a correct treatment of spin-orbit interactions and its application to topological phase transitions. Phys. Rev. B, 98:205137-1-17, 2018.

[88] K. Krieger, J. K. Dewhurst, P. Elliott, S. Sharma, and E. K. U. Gross. Laserinduced demagnetization at ultrashort time scales: predictions of TDDFT. $J$. Chem. Theor. Comput., 11:4870, 2015.

[89] The sign convention for the magnetization follows E. Engel and R. M. Dreizler, Density functional theory: An advanced course (Springer, Berlin, 2011).

[90] J. P. Perdew and Y. Wang. Accurate and simple analytic representation of the electron-gas correlation energy. Phys. Rev. B, 45:13244-9, 1992.

[91] J. P. Perdew. Climbing the ladder of density functional approximations. MRS Bulletin, 38:743-50, 2013.

[92] J. Kübler, K.-H. Höck, J. Sticht, and A. R. Williams. Density functional theory of non-collinear magnetism. J. Phys. F: Met. Phys., 18:469, 1988.

[93] L. M. Sandratskii. Noncollinear magnetism in itinerant-electron systems: theory and applications. Adv. Phys., 47:91, 1998. 
[94] K. Capelle, G. Vignale, and B. L. Györffy. Spin currents and spin dynamics in time-dependent density-functional theory. Phys. Rev. Lett., 87:106403, 2001.

[95] L. Kleinman. Density functional for noncollinear magnetic systems. Phys. Rev. $B, 59: 3314,1999$.

[96] F. G. Eich and E. K. U. Gross. Transverse spin-gradient functional for noncollinear spin-density-functional theory. Phys. Rev. Lett., 111:156401, 2013.

[97] F. G. Eich, S. Pittalis, and G. Vignale. Transverse and longitudinal gradients of the spin magnetization in spin-density-functional theory. Phys. Rev. B, 88:245102, 2013.

[98] S. Pittalis, G. Vignale, and F. G. Eich. U(1) $\times \mathrm{SU}(2)$ gauge invariance made simple for density functional approximations. Phys. Rev. B, 96:035141-1-8, 2017.

[99] M. I. Katsnelson and V. P. Antropov. Spin angular gradient approximation in the density functional theory. Phys. Rev. B, 67:140406, 2003.

[100] J. E. Peralta, G. E. Scuseria, and M. J. Frisch. Noncollinear magnetism in density functional calculations. Phys. Rev. B, 75:125119, 2007.

[101] G. Scalmani and M. J. Frisch. A new approach to noncollinear spin density functional theory beyond the local density approximation. J. Chem. Theor. Comput., 8:2193, 2012.

[102] I. W. Bulik, G. Scalmani, M. J. Frisch, and G. E. Scuseria. Noncollinear density functional theory having proper invariance and local torque properties. Phys. Rev. B, 87:035117, 2013. 
[103] S. Sharma, J. K. Dewhurst, C. Ambrosch-Draxl, S. Kurth, N. Helbig, S. Pittalis, S. Shallcross, L. Nordström, and E. K. U. Gross. First-principles approach to noncollinear magnetism: towards spin dynamics. Phys. Rev. Lett., 98:196405, 2007.

[104] S. Sharma, E. K. U. Gross, A. Sanna, and J. K. Dewhurst. Source-free exchangecorrelation magnetic fields in density functional theory. J. Chem. Theory Comput., 14:1247-53, 2018.

[105] C. A. Ullrich. Density-functional theory for systems with noncollinear spin: orbital-dependent exchange-correlation functionals and their application to the Hubbard dimer. Phys. Rev. B, 98:035140-1-13, 2018.

[106] C. A. Ullrich. (Spin-)density-functional theory for open-shell systems: exact magnetization density functional for the half-filled Hubbard trimer. Phys. Rev. A, 100:012516, 2019.

[107] J. E. Hirsch. Charge-density-wave to spin-density-wave transition in the extended Hubbard model. Phys. Rev. Lett., 53:2327-30, 1984.

[108] Y. Zhang and J. Callaway. Extended Hubbard model in two dimensions. Phys. Rev. B, 39:9397-404, 1989.

[109] M. S. Laad and D. K. Ghosh. Extended Hubbard model in two dimensions. J. Phys.: Condens. Matter, 3:9723-32, 1991.

[110] R. Schumann and D. Zwicker. The Hubbard model extended by nearestneighbor Coulomb and exchange interaction on a cubic cluster - rigorous and exact results. Ann. Phys. (Berlin), 522:419-39, 2010. 
[111] D. J. Carrascal, J. Ferrer, J. C. Smith, and K. Burke. The Hubbard dimer: a density functional case study of a many-body problem. J. Phys.: Condens. Matter, 27:393001, 2015.

[112] R. Strack and D. Vollhardt. Hubbard model with nearest-neighbor and bondcharge interaction: exact ground-state solution in a wide range of parameters. Phys. Rev. Lett., 70:2637-40, 1993.

[113] K. Capelle and V. L. Campo Jr. Density functionals and model hamiltonians: Pillars of many-particle physics. Phys. Rep., 528:91-159, 2013.

[114] D. S. Jensen and A. Wasserman. Numerical methods for the inverse problem of density functional theory. Int. J. Quant. Chem., 118:e25425-1-29, 2017.

[115] W. H. Press, S. A. Teukolsky, W. T. Vetterling, and B. P. Flanner. Numerical recipes 3rd edition: the art of scientific computing. Cambridge University Press, Cambridge, 2007.

[116] T. A. Kaplan. Single-band Hubbard model with spin-orbit coupling. Z. Phys. B: Condens. Matter, 49:313-7, 1983.

[117] S. Ghassemi Tabrizi, A. V. Arbuznikov, and M. Kaupp. Hubbard trimer with spin-oprbit coupling: Hartree-Fock solutions, (non)collinearity, and anisotropic spin Hamiltonian. J. Phys. Chem., 123:2361-78, 2019.

[118] K. S. Singwi, A. Sjölander, M. P. Tosi, and R. H. Land. Electron correlations at metallic densities. Phys. Rev., 176:589-99, 1968.

[119] G. F. Giuliani and G. Vignale. Quantum Theory of the Electron Liquid. Cambridge University Press, Cambridge, 2005. 
[120] Yair Kurzweil and Roi Baer. Adapting approximate-memory potentials for time-dependent density functional theory. Phys. Rev. B, 77:085121-1-8, 2008.

[121] Yair Kurzweil and Martin Head-Gordon. Improving approximate-optimized effective potentials by imposing exact conditions: Theory and applications to electronic statics and dynamics. Phys. Rev. A, 80:012509-1-12, 2009.

[122] The singlet/triplet character of the exact wave function follows from the exact diagonalization, which is carried out in a basis of noninteracting singlet and triplet states.

[123] C. R. Jacob and M. Reiher. Spin in density-functional theory. Int. J. Quantum Chem., 112:3661-84, 2012.

[124] M. Fuchs, Y.-M. Niquet, X. Gonze, and K. Burke. Describing static correlation in bond dissociation by Kohn-Sham density functional theory. J. Chem. Phys., 122:094116-1-13, 2005.

[125] A. J. Cohen, P. Mori-Sánchez, and W. Yang. Challenges for density functional theory. Chem. Rev., 112:289-320, 2012.

[126] N. Q. Su, C. Li, and W. Yang. Describing strong correlation with fractionalspin correction in density functional theory. Proc. Natl. Acad. Sci., 115:9678-83, 2018 .

[127] Andreas Görling. Hierarchies of methods towards the exact Kohn-Sham correlation energy based on the adiabatic-connection fluctuation-dissipation theorem. Phys. Rev. B, 99:235120-1-20, 2019. 
[128] L. Balents. Spin liquids in frustrated magnets. Nature, 464:199-208, 2010.

[129] Mircea Trif, Filippo Troiani, Dimitrije Stepanenko, and Daniel Loss. Spin electric effects in molecular antiferromagnets. Phys. Rev. B, 82:045429, 2010.

[130] Yuanzuo Li, Yu Sun, Yongqing Li, and Fengcai Ma. Electronic and optical properties of neutral and charged meh-ppv. Computational Materials Science, $39(3): 575-579,2007$.

[131] Gang Qian, Bo Dai, Min Luo, Dengbin Yu, Jie Zhan, Zhiqiang Zhang, Dongge Ma, and Zhi Yuan Wang. Band gap tunable, donor-acceptor-donor chargetransfer heteroquinoid-based chromophores: Near infrared photoluminescence and electroluminescence. Chemistry of Materials, 20(19):6208-6216, 2008.

[132] Wenchao Zhao, Deping Qian, Shaoqing Zhang, Sunsun Li, Olle Inganäs, Feng Gao, and Jianhui Hou. Fullerene-free polymer solar cells with over 11\% efficiency and excellent thermal stability. Advanced Materials, 28(23):4734-4739, 2016.

[133] Massimiliano Cavallini, Massimo Facchini, Cristiano Albonetti, and Fabio Biscarini. Single molecule magnets: from thin films to nano-patterns. Phys. Chem. Chem. Phys., 10:784-793, 2008.

[134] Constantinos J. Milios, Stergios Piligkos, and Euan K. Brechin. Ground state spin-switching via targeted structural distortion: twisted single-molecule magnets from derivatised salicylaldoximes. Dalton Trans., pages 1809-1817, 2008.

[135] A. A. Zvyagin. New physics in frustrated magnets: Spin ices, monopoles, etc. Low Temp. Phys., 39:901, 2013. 
[136] A. M. Hallas, A. M. Arevalo-Lopez, A. Z. Sharma, T. Munsie, J. P. Attfield, C. R. Wiebe, and G. M. Luke. Magnetic frustration in lead pyrochlores. Phys. Rev. B, 91:104417, Mar 2015.

[137] Michael L. Baker, Grigore A. Timco, Stergios Piligkos, Jennifer S. Mathieson, Hannu Mutka, Floriana Tuna, Piotr Kozłowski, Michał Antkowiak, Tatiana Guidi, Tulika Gupta, Harapriya Rath, Robert J. Woolfson, Grzegorz Kamieniarz, Robin G. Pritchard, Høgni Weihe, Leroy Cronin, Gopalan Rajaraman, David Collison, Eric J. L. McInnes, and Richard E. P. Winpenny. A classification of spin frustration in molecular magnets from a physical study of large odd-numbered-metal, odd electron rings. Proceedings of the National Academy of Sciences, 109(47):19113-19118, 2012.

[138] M. J. Frisch, G. W. Trucks, H. B. Schlegel, G. E. Scuseria, M. A. Robb, J. R. Cheeseman, G. Scalmani, V. Barone, G. A. Petersson, H. Nakatsuji, X. Li, M. Caricato, A. V. Marenich, J. Bloino, B. G. Janesko, R. Gomperts, B. Mennucci, H. P. Hratchian, J. V. Ortiz, A. F. Izmaylov, J. L. Sonnenberg, D. Williams-Young, F. Ding, F. Lipparini, F. Egidi, J. Goings, B. Peng, A. Petrone, T. Henderson, D. Ranasinghe, V. G. Zakrzewski, J. Gao, N. Rega, G. Zheng, W. Liang, M. Hada, M. Ehara, K. Toyota, R. Fukuda, J. Hasegawa, M. Ishida, T. Nakajima, Y. Honda, O. Kitao, H. Nakai, T. Vreven, K. Throssell, J. A. Montgomery, Jr., J. E. Peralta, F. Ogliaro, M. J. Bearpark, J. J. Heyd, E. N. Brothers, K. N. Kudin, V. N. Staroverov, T. A. Keith, R. Kobayashi, J. Normand, K. Raghavachari, A. P. Rendell, J. C. Burant, S. S. Iyengar, J. Tomasi, M. Cossi, J. M. Millam, M. Klene, C. Adamo, R. Cammi, J. W. 
Ochterski, R. L. Martin, K. Morokuma, O. Farkas, J. B. Foresman, and D. J. Fox. Gaussian 16 Revision A.03, 2016. Gaussian Inc. Wallingford CT.

[139] M. Valiev, E.J. Bylaska, N. Govind, K. Kowalski, T.P. Straatsma, H.J.J. Van Dam, D. Wang, J. Nieplocha, E. Apra, T.L. Windus, and W.A. de Jong. Nwchem: A comprehensive and scalable open-source solution for large scale molecular simulations. Computer Physics Communications, 181(9):1477 - 1489, 2010.

[140] Baojing Zhou, Yan Alexander Wang, and Emily A. Carter. Transferable local pseudopotentials derived via inversion of the Kohn-Sham equations in a bulk environment. Phys. Rev. B, 69:125109, 2004. 


\section{VITA}

Edward A. Pluhar III was born across the river from St. Louis, Missouri, in the village of Alton, Illinois, in 1991. Attending Metro-East Lutheran High School, he took his first physics class with Dr. Becky Hutchinson, sparking an interest in the physical sciences that began his journey into physics research. After graduating with honors from MELHS, Edward worked under the supervision of Dr. Eric Martell at Millikin University in Decautur, Illinois. While there, Eddie was exposed to theoretical physics research, presenting his senior capstone project as a poster presentation at American Physical Society's April Meeting, and graduated with a B.S. in physics with a minor in mathematics. Prior to graduation, Edward was also selected by the physics faculty at Millikin as the outstanding physics major for the 2011-2012 school year.

Upon the completion of his undergraduate studies, Edward briefly pursued a technical sales role in industrial electrical equipment. Determined to continue his education and grow intellectually, he returned to academia in 2014, matriculating at the University of Missouri in Columbia, Missouri, to resume his physics educcation. In the spring of 2015, he joined Dr. Carsten Ullrich's research group to pursue the study of theoretical physics using Density-functional Theory. While at the University of Missouri, Edward received his Masters of Science in physics upon the completion of his qualifying and comprehensive exams during his third year.

While wrapping up his doctorate in 2019-2020, Eddie was awarded an intern role with the quantitative strategies team at Veterans United Home Loans. After completing his internship in the Summer of 2020, Edward married Lainey Poor on 
June 6th, 2020, in the height of the age of COVID-19. Upon successful defense of his thesis, Edward accepted a job offer with the Teacher Retirement System of Texas in their quantitative investments division. 